Copyright

by

Shaunak Ghosh

2013 
The Thesis Committee for Shaunak Ghosh

certifies that this is the approved version of the following thesis:

\title{
Multiple suppression in the $t-x-p$ domain
}

\author{
APPROVED BY \\ SUPERVISING COMMITTEE:
}

Sergey Fomel, Supervisor

Stephen Grand

Bob Hardage 
Multiple suppression in the $t-x-p$ domain

by

Shaunak Ghosh, B.S.,M.S.

\author{
THESIS \\ Presented to the Faculty of the Graduate School of \\ The University of Texas at Austin \\ in Partial Fulfillment \\ of the Requirements \\ for the Degree of \\ MASTER OF SCIENCE IN GEOLOGICAL SCIENCES
}

THE UNIVERSITY OF TEXAS AT AUSTIN

December 2013 
Dedicated to my parents. 


\section{Acknowledgments}

I would like to thank my advisor, Prof. Sergey Fomel, for his guidance and academic and financial support throughout the course of my graduate program. Being in his group enabled me to learn about the technical horizon in the field of geophysics that will benefit me in both the present and future. I would also like to thank my committee members, Dr. Bob Hardage and Dr. Stephen Grand, for serving on my thesis committee and giving valuable feedback. I would also like to extend my thanks to Karl Schleicher, Alexander Klokov and Prof. Paul Stoffa for valuable discussions in geophysics and sharing knowledge of what it is like to work for the industry. I would like to thank Vladimir Bashkardin for initial help with learning Madagascar. I thank Parvaneh Karimi and Luke Decker for their friendship. I would like to thank Philip Guerrero who I believe is one of the two most important people in the academic life of a graduate student at the Jackson School, along with one's advisor. Finally, I would like to thank my family for their continual encouragement and support.

Shaunak Ghosh

The University of Texas at Austin

December 2013 


\title{
Multiple suppression in the $t-x-p$ domain
}

\author{
Shaunak Ghosh, M.S.Geo.Sci. \\ The University of Texas at Austin, 2013
}

Supervisor: Sergey Fomel

Multiples in seismic data pose serious problems to seismic interpreters for both AVO studies and interpretation of stacked sections. Several methods have been practiced with varying degrees of success to suppress multiples in seismic data. One family of velocity filters for demultiple operations using Radon transforms traditionally face challenges when the water column is shallow. Additionally, the hyperbolic Radon Transform can be computationally expensive. In this thesis, I introduce a novel multiple suppression technique in the $t-x$ - $p$ domain, where $p$ is the local slope of seismic events, that aims at tackling some of the aforementioned limitations, and discuss the advantages and scope of this approach. The technique involves essentially two steps: the decomposition part and the suppression part. Common Mid-Point (CMP) gathers are taken and transformed from the original $t$ - $x$ space to the extended $t-x-p$ space and eventually to the $t_{0}-x-p$ space, where $t_{0}$ is the zero offset traveltime. Multiplication of the gather in the extended space with Gaussian tapering filters, formed using the difference of the powers of the the picked primary velocities and intrinsically calculated velocities in terms of $t_{0}, x$ and $p$ using analytical relations, and stacking along the $p$ axis produces gathers with multiples suppressed. 


\section{Table of Contents}

Acknowledgments $\quad$ v

Abstract vi vi

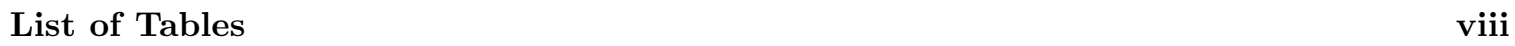

List of Figures $\quad$ ix

\begin{tabular}{lll}
\hline Chapter 1. Introduction & 1
\end{tabular}

\begin{tabular}{lll}
\hline Chapter 2. & Review of multiple suppression methods & 11
\end{tabular}

\begin{tabular}{lll}
\hline Chapter 3. & Multiple suppression in the $t-x-p$ domain* & 37
\end{tabular}

$\begin{array}{lll}\text { Chapter 4. } & \text { Multiple attenuation of the Viking Graben data set } & 62\end{array}$

\begin{tabular}{lll}
\hline Chapter 5. & Discussion and conclusions & 105
\end{tabular}

\begin{tabular}{lr}
\hline Bibliography & 108
\end{tabular} 
List of Tables 


\section{List of Figures}

1.1 Water-bottom multiples of (a) First order. (b) Second order. . . . . . . . . . . . . 4

$1.2 \quad$ Peg-leg multiples of (a) First order. (b) Second order. . . . . . . . . . . . . . . . 5

$1.3 \quad$ Intra-bed multiples of (a) First order. (b) Second order. . . . . . . . . . . . . . 6

$1.4 \quad$ Inter-bed multiples of (a) First order. (b) Second order. . . . . . . . . . . . . . 6

1.5 Selected shot gathers from the Viking-Graben data set. . . . . . . . . . . . . . . . 9

1.6 Near offset section for line 12 of the Viking Graben data set. The record is strongly contaminated by multiples. $\ldots \ldots \ldots \ldots \ldots \ldots \ldots$

$2.1 \quad$ A flowchart for predictive deconvolution using prediction filters. (Yilmaz, 2001) . . . 13

$2.2 \quad$ A flowchart for predictive deconvolution using prediction error filters. (Yilmaz, 2001) 14

2.3 (a) Original CMP gather, (b) NMO corrected gather with primary velocity. . . . . . 23

2.4 (a) NMO corrected gather with primary velocity , (b) NMO corrected gather with

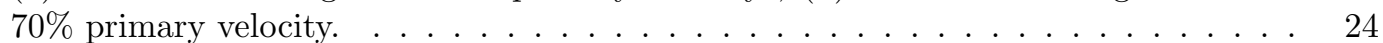

2.5 (a) Real part of the data in the $f k$ domain, (b) Real part of the data in the $f k$

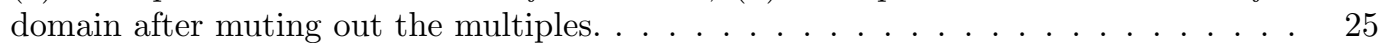

2.6 (a) Restored gather after NMO and inverse NMO , (b) Demultipled gather using $f k$ filtering and (c) Subtracted multiples (difference between (a) and (b)). . . . . . . . 26

2.7 (a) Velocity scan before demultiple, (b) Velocity scan after demultiple. . . . . . . . 27

\begin{tabular}{|lll}
2.8 & (a) Data in the $\tau-p$ domain, (b) Data in the $\tau-p$ domain after muting out the multiples. 32
\end{tabular}

2.9 (a) Restored gather after NMO and inverse NMO , (b) Demultipled gather using Parabolic Radon and (c) Subtracted multiples (difference between (a) and (b)). . . . 33

2.10 (a) Velocity scan before demultiple, (b) Velocity scan after demultiple. . . . . . . . 34

$3.1 \quad$ Synthetic CMP gather in $t$ - $x$ domain (a) and $f$-x domain (b,real part). . . . . . . . 40

3.2 The CMP gather in the extended $f$ - $x-p$ domain (a, real part) and $t-x-p$ domain (b).

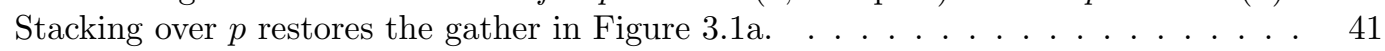

$3.3 \quad$ Schematic illustration of LTFK deomposition. . . . . . . . . . . . . . . . . . . . 44

$3.4 \quad$ Schematic illustration of LXFK deomposition. . . . . . . . . . . . . . . . . . . . 45 
3.5 The CMP gather in the extended $t-x-p$ domain. Stacking over $p$ restores the gather

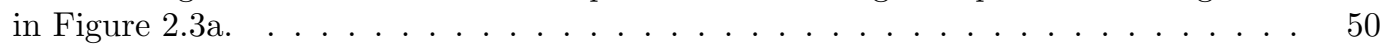

$3.6 \quad$ (a) Reconstructed CMP gather, (b) Difference with original gather. . . . . . . . . . . 51

3.7 (a) Gather after demultiple, (b) Difference with original gather. . . . . . . . . . . . . 52

$3.8 \quad$ NMO corrected gather with picked primary velocity (a) before demultiple and (b)

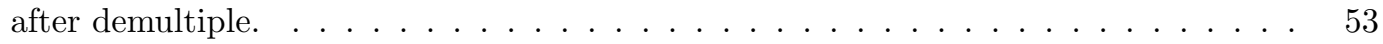

3.9 (a) Velocity scan before demultiple, (b) Velocity scan after demultiple. . . . . . . . 54

3.10 (a) Original CMP gather. (b) NMO corrected gather with picked primary velocities 56

3.11 CMP gather in the extended $t$ - $x$ - $p$ domain $\ldots \ldots \ldots \ldots \ldots$. . . . . . . . . 57

3.12 (a) Original gather. (b) Gather after multiple suppression. (c) Residual (filtered out

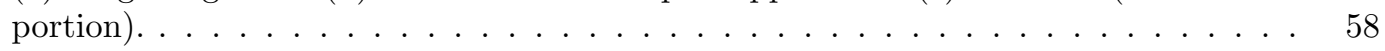

3.13 Velocity scan (a) before multiple attenuation. (b) after multiple attenuation. . . . . 59

3.14 (a) Original CMP gather. (b) Gather after ground roll suppression. (c) Residual

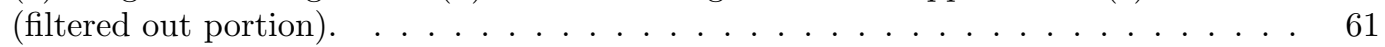

4.1 Map of the Viking Graben Area, modified from Glennie $[(1990) . \ldots \ldots$. . . . . . . . . 64

\begin{tabular}{|lll|ll|}
\hline 4.2 & Stratigraphic column of the northern North Sea reservoir rocks (Glenni®|(1990)). & . . & 65 \\
\hline
\end{tabular}

$4.3 \quad$ Migrated section. The major unconformity at the sase of the Cretaceous is located at 1.97s at Well A and 2.46s in Well B (Madiba and McMechan (2003)). $\ldots . . .666$

4.4 Shots $300,400,500,600$ and 700 from the data line. . . . . . . . . . . . 68

$4.5 \quad$ Near offset section for the entire $25 \mathrm{~km}$ Viking Graben line. . . . . . . . . . . . . . . 69

4.6 CMPs 201, 401, 601, 801, 1001 and 1201 from the data line. . . . . . . . . . . 70

4.7 Velocity scans with picked primary velocities for CMPs 201, 401, 601, 801, 1001 and 1201 from the data line. Though in some of the gathers, the primaries for the deeper parts are incomprehensible, velocity after demultiple reveals reasonable accuracy of

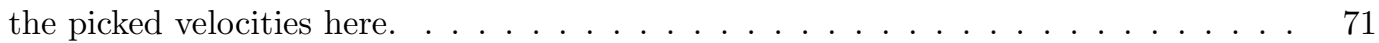

4.8 NMO corrected gathers for CMPs $201,401,601,801,1001$ and 1201 from the data

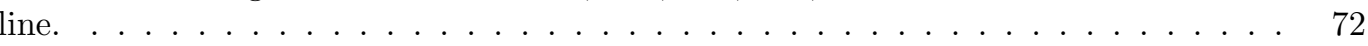

4.9 Picked primary velocity for the entire line. $\ldots \ldots \ldots \ldots \ldots$

4.10 Normal Moveout (NMO) stack with picked primary velocity for the entire line for the

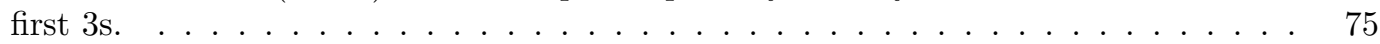

4.11 Normal Moveout (NMO) stack with water velocity for the entire line for the first 3s. 76

4.12 CMPs 201, 401, 601, 801, 1001 and 1201 from the data line after demultiple. . . . . 79 
4.13 Velocity scans for CMPs 201, 401, 601, 801, 1001 and 1201 from the data line after

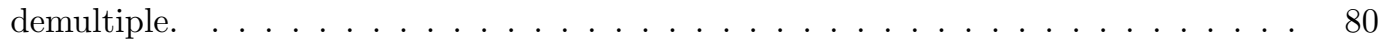

4.14 Normal Moveout (NMO) stack with picked primary velocity for the entire line for the

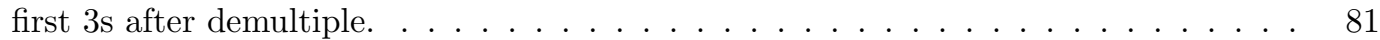

4.15 Difference stack before and after demultiple in $t$-x-p domain. . . . . . . . . . . . 82

4.16 Normal Moveout (NMO) stack with water velocity for the entire line for the first 3s

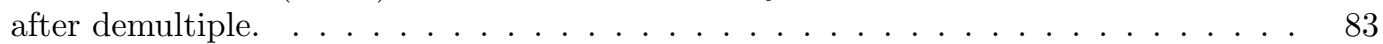

4.17 Normal Moveout (NMO) stack with picked primary velocity for the entire line for the first 3s before SRME. $\ldots \ldots \ldots \ldots \ldots$. . . . . . . . . . . . . . . . . .

4.18 Normal Moveout (NMO) stack with picked primary velocity for the entire line for the first 3 s after SRME. . . . . . . . . . . . . . . . . . . . . . . . 87

4.19 Difference section before and after SRME. . . . . . . . . . . . . . . . . . . . 88

4.20 Normal Moveout (NMO) stack with water velocity for the entire line for the first $3 \mathrm{~s}$

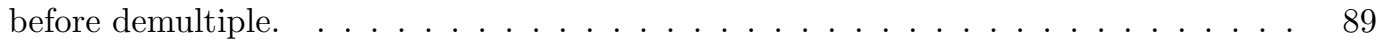

4.21 Normal Moveout (NMO) stack with water velocity for the entire line for the first $3 \mathrm{~s}$

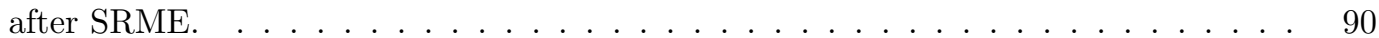

4.22 Near offset section before SRME. . . . . . . . . . . . . . . . . . . . . . . 91

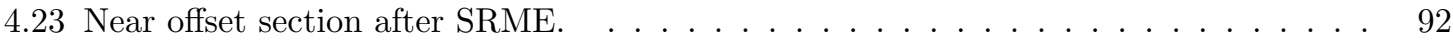

4.24 Near offset difference section after SRME. . . . . . . . . . . . . . . . . . . . . . . . 93

4.25 Velocity scans for CMPs 201, 401,601,801,1001 and 1201 after SRME. . . . . . . 94

4.26 CMPs $201,401,601,801,1001$ and 1201 before $t$ - $x$ - $p$ filtering. . . . . . . . . . . 97

4.27 CMPs $201,401,601,801,1001$ and 1201 after $t-x$ - $p$ filtering. . . . . . . . . . . . 98

4.28 Normal Moveout (NMO) stack with picked primary velocity for the entire line for the

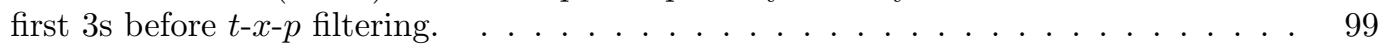

4.29 Normal Moveout (NMO) stack with picked primary velocity for the entire line for the first 3 s after $t-x-p$ filtering. $\ldots \ldots \ldots \ldots \ldots$

4.30 Difference section before and after $t-x-p$ filtering. . . . . . . . . . . . . . . . 101

4.31 Normal Moveout (NMO) stack with water velocity for the entire line for the first $3 \mathrm{~s}$

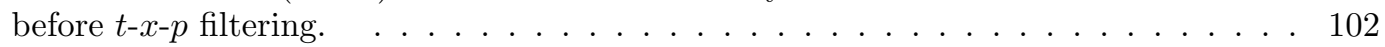

4.32 Normal Moveout (NMO) stack with water velocity for the entire line for the first $3 \mathrm{~s}$

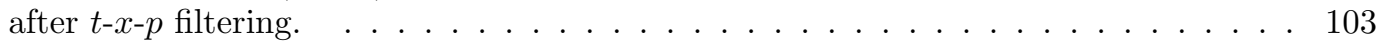

4.33 Velocity scans for CMPs $201,401,601,801,1001$ and 1201 after $t$ - $x$ - $p$ filtering. . . . 104 


\section{Chapter 1}

\section{Introduction}

This chapter explains how the subsurface of the earth is imaged with the aid of seismic imaging technology, what multuples acquired in seismic data are, the different kinds of multiples and the structure of this thesis.

Hydrocarbons are formed as a result of organic material trapped within the earth's sub-surface being subject to suitable Pressure-Temperature (P-T) conditions due to the ongoing process of burial. This hydrocarbon after being formed undergoes expulsion and then often migrates as a result of compaction till it is entrapped within a potential trap, i.e, it is contained by a porous host rock and laid over by an impermeable cap rock. In order to exploit hydrocarbon reserves efficiently, a clear, distinct and interpretable image of the subsurface is necesary for the seismic interpreter to identify potential hydrocarbon traps. Generally, seismic reflection surveys are conducted to accomplish this task, wherein a seismic wave is sent into the earth. Seismic energy is generated via a dynamite or vibroseis source on land and air guns in marine surveys. Due to the process of sedimentation, there is an impedance (product of velocity and density) contrast between layers of sediments due to the varying elastic properties of various sedimentary layers. When the downgoing wave propagates and comes across such an interface of impedance change, a part of the wave gets reflected and another part gets transmitted. The reflected wavefield is recorded at the surface with the help of receiver 
arrays and is used to image the sub-surface.

The method of seismic prospecting can be divided into four broad (and sometimes overlapping) categories: acquisition, pre-processing, processing and imaging. During the data acquisition stage, some of the recorded wavefields undergo only one reflection before being recorded at the receivers. These reflections are referred to as primary reflections. Some other reflections undergo two or more reflections at one or more interfaces before being recorded at the receivers. Such reflections are called multiple reflections or multiples. Multiples need to be removed during the pre-processing and processing stages. If multiples are not removed, then they often interfere with primary reflections in pre-stack and stacked seismic data and make interpretation of sub-surface features difficult. The next section explains in more detail what multiples are and the different kinds of multiples that exist.

\section{MULTIPLES}

Multiples are events that have undergone multiple reflections in a seismic experiment before being recorded at a receiver. i.e, instead of a seismic wave propagating from a source, getting reflected once at a geologic interface and eventually being recorded at a receiver, they undergo multiple reflections at one or more of these interfaces.

Multiples are present in both marine and land seismic data, but are more of a concern usually in marine surveys. The higher the impedance contrast (product of density and velocity) between two layers, the greater is the amplitude of the reflected wave. In marine surveys, the interface between the water and water bottom has a very high impedance contrast and the water-air interface 
also acts almost like a free surface. Therefore, marine data sets can contain several orders of water bottom multiples, along with other kinds of multiples (discussed in a separate section).

Since for exploration and production, generally only reflected primary energy is used, multiples contaminate data and act as a deterrent from obtaining a proper image of the sub-surface. The problem is compounded by the fact that multiples can have higher energy than the primaries from deeper reflectors that they mask or interfere with.

\section{DIFFERENT TYPES OF MULTIPLES}

\section{Water bottom multiples}

Water bottom multiples are multiples whose raypaths lie entirely in the water column. These multiples can undergo two or more reflections during their entire path between source and receiver. Since the impedance contrast at the water bottom is very high, these multiples can have very high amplitudes compared to the primary reflections that they interfere with. Figure 1.1 (borrowed from Yilmaz (2001)) shows first and second order water-bottom multiples. The horizon where the

grey part starts represents the water-bottom. In the first figure, the wavefield propagates from the source, gets reflected at the water-bottom, propagates up to the water surface and then reflects off the water bottom again before being finally recorded at the receiver. This is the first multiple that can be generated and is caused by two reflections off the water-bottom. Hence, it is called the first-order water bottom multiple. The second figure shows that the wavefield gets reflected thrice at the water-bottom before being recorded at the receiver. Hence it is called a second-order water bottom multiple. 

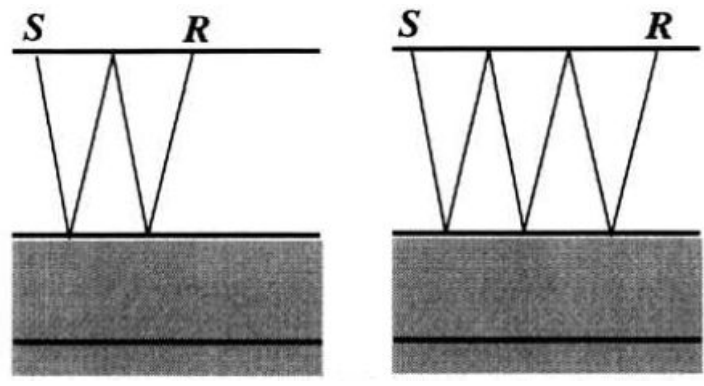

Figure 1.1: Water-bottom multiples of (a) First order. (b) Second order. chapter $1 /$ multiples waterbottom

\section{Peg-leg multiples}

Peg-leg multiples are defined in different ways by various authors. I use the definition of peg-leg multiples as waves that undergo one or more reflections at a sedimentary surface and one or more reflections at near surface.

Figure 1.2 (borrowed from Yilmaz (2001)) shows first and second order peg-leg multiples. The first figure (first-order) shows a multiple that is reflected once at a sedimentary surface and once at the near surface, while the second figure (second-order) shows a multiple that is reflected twice at a sedimentary surface and once at the near surface before being recorded at the receiver. 

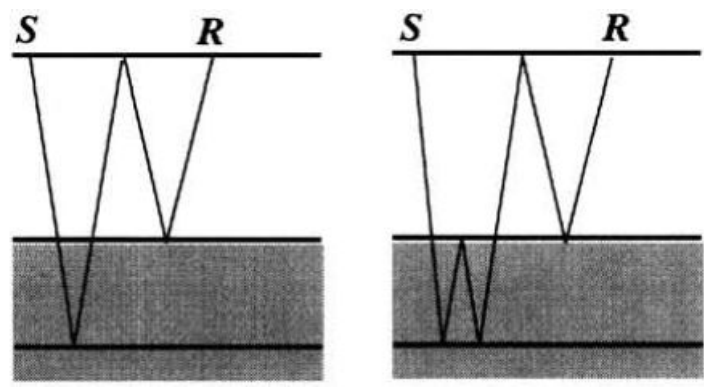

Figure 1.2: Peg-leg multiples of (a) First order. (b) Second order. chapter1/multiples pegleg

\section{Intra-bed multiples}

Intra-bed multiples are multiples that undergo multiple reflections inside the same sedimentary layer or bed before being recorded at a receiver. They are also called short-path multiples. They are rarely observed in seismic data and are often identified only when seismic data is being interpreted with the accompaniment of well logs. The reason for their rare occurence is the fact that the impedence contrast at seismic interfaces are typically much less than those at the free surfaces.

Figure 1.3 (borrowed from Yilmaz (2001)) shows first and second order intra-bed multiples. The wavefield gets reflected once and twice within the bed formed by the water bottom and the next interface before being recorded at the receiver, generating first and second order intra-bed multiples respectively. 

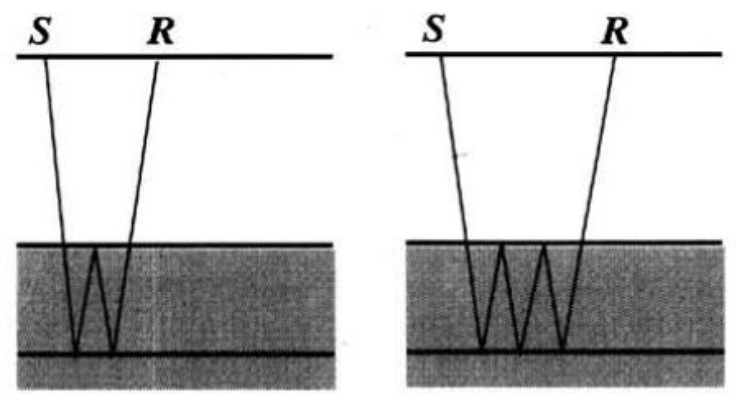

Figure 1.3: Intra-bed multiples of (a) First order. (b) Second order. chapter1/multiples intrabed

\section{Inter-bed multiples}

Inter-bed multiples are multiples that undergo multiple reflections between two or more separate sedimentary layers before being recorded at the receiver.

Figure 1.4 (borrowed from Yilmaz (2001)) shows first and second order inter-bed multiples. The
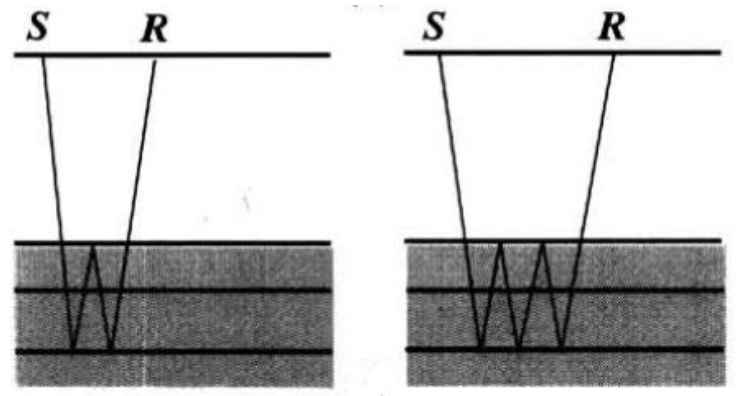

Figure 1.4: Inter-bed multiples of (a) First order. (b) Second order. chapter1/multiples interbed

wavefield undergoes multiple reflections within the two sedimentary reflections beneath the water 
column before being recorded at the receiver. In the first figure, the wavefield gets reflected once within the two aforementioned layers before being recorded at the receiver, giving rise to first-order water bottom multiples, while in the second figure, the wavefield gets reflected twice within the two aforementioned layers before being recorded at the receiver to generate second-order inter-bed multiples.

Over the years, several attempts have been made with varying degrees of success to suppress multiples in seismic data (Weglein and Dragoset, 2005, Verschuur, 2012). However, it still poses a very challenging task for processing geophysicists. The main goal of this thesis is to describe a novel technique based on velocity/slope discrimination (Ghosh and Fomel, 2012) and its application to a field data set.

This thesis is divided into five chapters. Chapter 1 is the introduction that explains what multiples are and the different kinds of multiples. Chapter 2 describes the basis on which multiples are identified and separated and the popular multiple suppression techniques. Chapter 3 describes multiple suppression in the $t-x-p$ domain and demonstrates the effectiveness of the method with synthetic and real data examples. Chapter 4 shows the application of the method on a real data set, line 12 of the the Viking Graben data set, acquired in the North Sea by Mobil Oil Corporation. This data was publicly made available in 1994 to support research for processing and inversion of seismic data (Keys and Foster, 1998). Figure 1.5 show some shot gathers from the line while Figure 1.6 shows the near offset section that clearly shows the presence of multiples. These figures are re-visited 
in Chapter 4 in the section that describes the Viking Graben data set. This chapter shows both pre-stack and stacked results to demonstrate the effectiveness of the new method. Chapter 5 draws conclusions and recommends future work and possible work flows that could be used for different multiple suppression cases. 

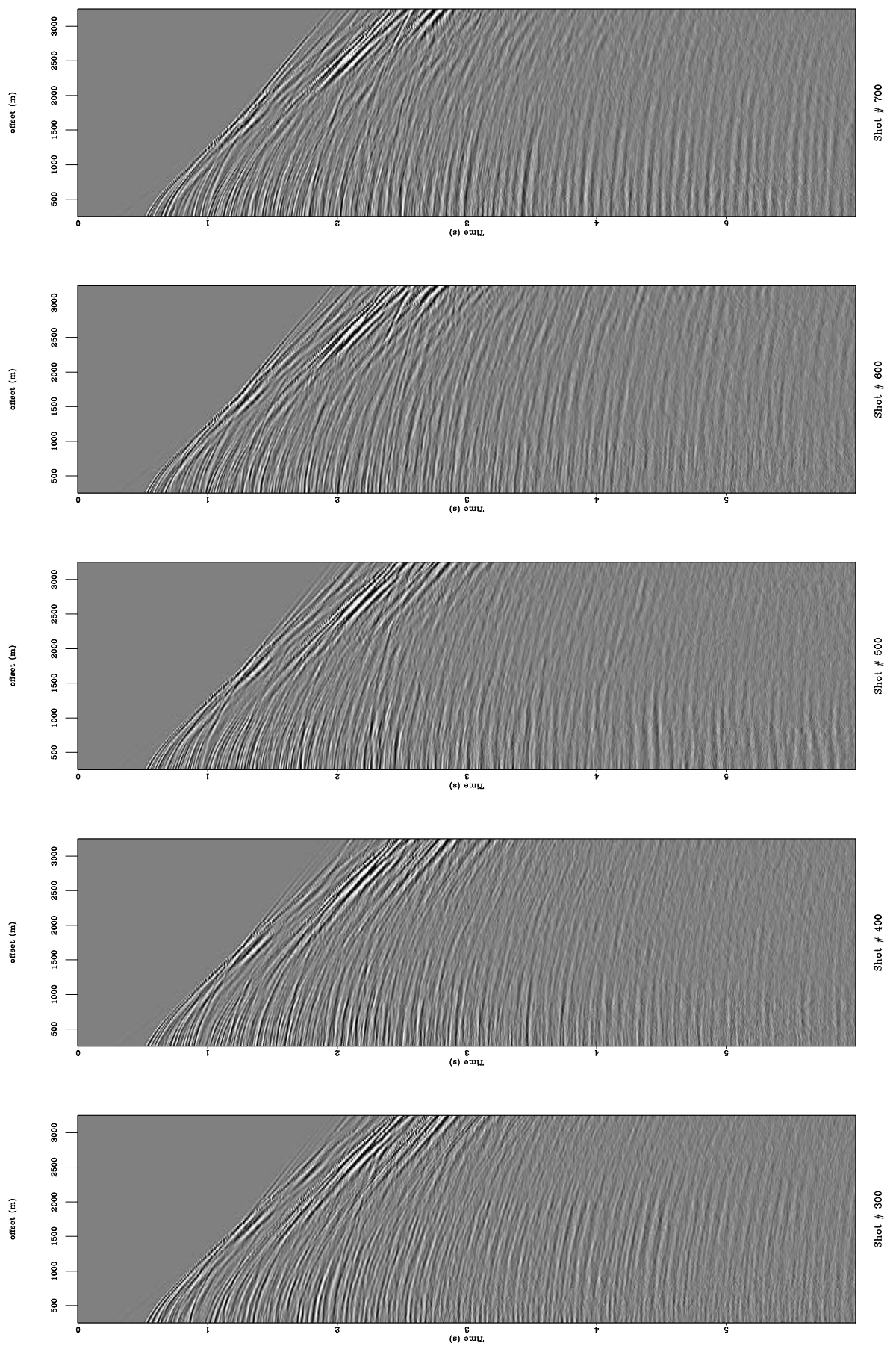

Figure 1.5: Selected shot gathers from the Viking-Graben data set. chapter1/multiples someshotgathers 


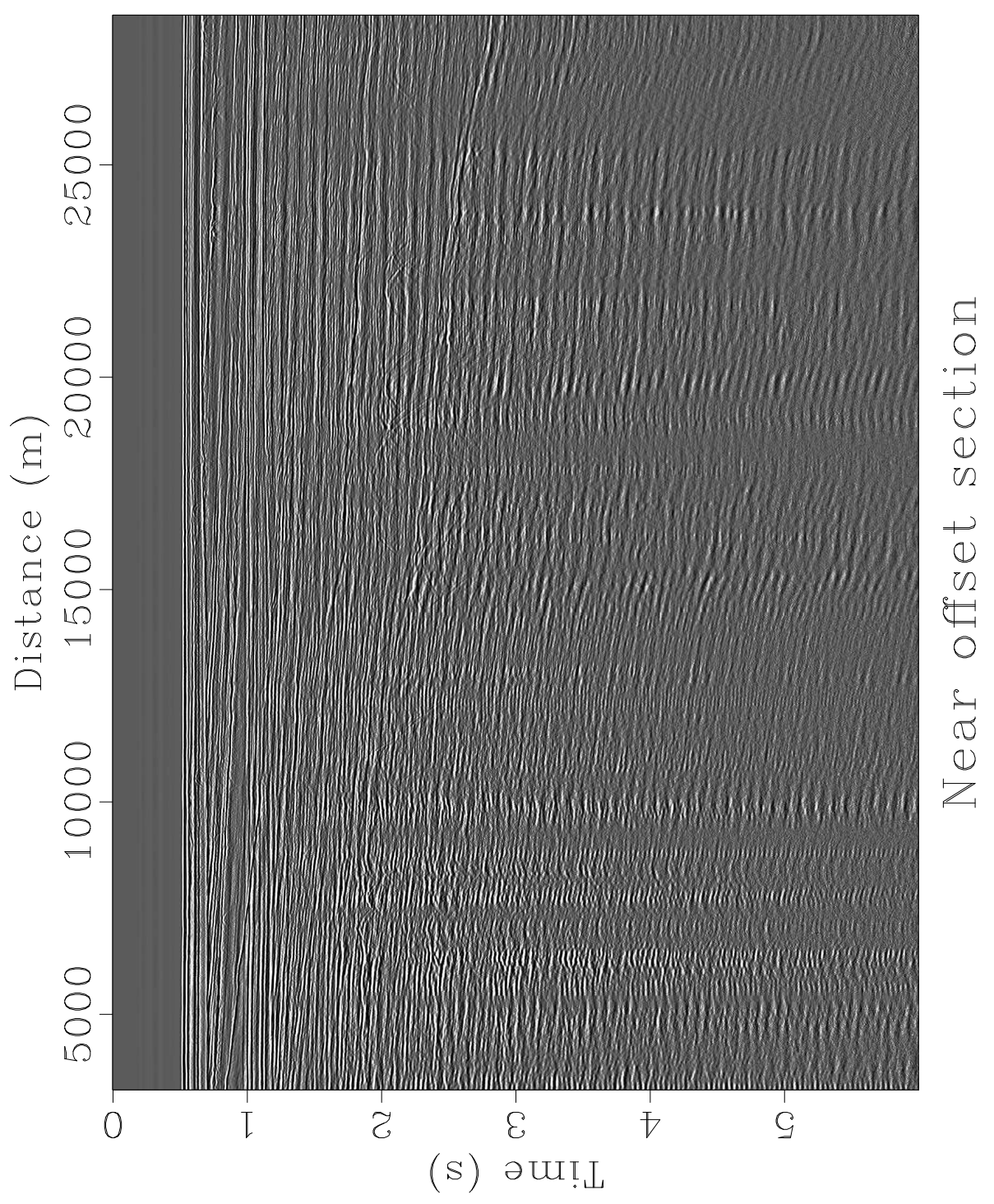

Figure 1.6: Near offset section for line 12 of the Viking Graben data set. The record is strongly contaminated by multiples. chapter1/multiples nearoffset 


\section{Chapter 2}

\section{Review of multiple suppression methods}

Multiples are conventionally suppressed either on the basis of their periodicity or velocity discrimination with primaries. The methods that focus on exploiting periodicty of multiples are:

1. Predictive deconvolution in the $\tau-p$ domain (Taner, 1980, Alam and Austin, 1981, Treitel et al. 1982, Tatham, 1984).

2. Surface related multiple elimination (SRME) (Verschuur et al., 1988, Dragoset, 1998, 1999).

3. Wave equation demultiple (WEREM) (Lokshtanov, 2000).

The methods that rely on velocity discrimination are:

1. $f$ - $k$ filtering (Meek and Linvelle, 1993).

2. $\tau$-p filtering (Tatham, 1984).

3. Parabolic Radon transform (Hampson, 1986, 1987).

4. Hyperbolic Radon transform (Foster and Mosher, 1992).

5. $t$ - $x$ - $p$ filtering (Ghosh and Fomel, 2012). 


\section{PREDICTIVE DECONVOLUTION}

Predictive decomvolution has proven to be a useful technique to predict and eliminate multiples from seismic data. It involves, given an input $x(t)$, we want to predict its value at some future time $(t+\alpha)$, where $\alpha$ is the prediction lag. Robinson and Treitel (1980) showed that the prediction filter used to estimate $x(t+\alpha)$ can be calculated by solving the following matrix equation:

$$
\left(\begin{array}{ccccc}
r_{0} & r_{1} & r_{2} & \ldots \ldots & r_{n-1} \\
r_{1} & r_{0} & r_{1} & \ldots \ldots & r_{n-2} \\
r_{2} & r_{1} & r_{0} & \ldots \ldots & r_{n-3} \\
\vdots & \vdots & \vdots & \ddots & \vdots \\
r_{n-1} & r_{n-2} & r_{n-3} & \ldots \ldots & r_{0}
\end{array}\right)\left(\begin{array}{c}
a_{0} \\
a_{1} \\
a_{2} \\
\vdots \\
a_{n-1}
\end{array}\right)=\left(\begin{array}{c}
r_{\alpha} \\
r_{\alpha+1} \\
r_{\alpha+2} \\
\vdots \\
r_{\alpha+n-1}
\end{array}\right)
$$

where, $r_{i}$ is the $i t h$ lag of the autocorrelation of the data $x(t), a_{i}$ is the $i t h$ element of the prediction filter of length $n$ and $m$ is the desired delay. In the event of constant perodicity of multiples, the multiples can be predicted by convolving the prediction filter with the data and by applying the desired lag. Thereafter, the multiples can be subtracted from the input to obtain data with multiples suppressed.

There are two ways to doing predictive deconvolution:

1. The prediction filter $\left(a_{0}, a_{1}, a_{2}, a_{3}, \ldots, a_{n-1}\right)$ can be calculated and applied on the input series.

2. The prediction error filter $\left(1,0,0,0, \ldots, 0,-a_{0},-a_{1},-a_{2}, \ldots,-a_{n-1}\right)$ can be designed and convolved with the input series to get demultipled data. 


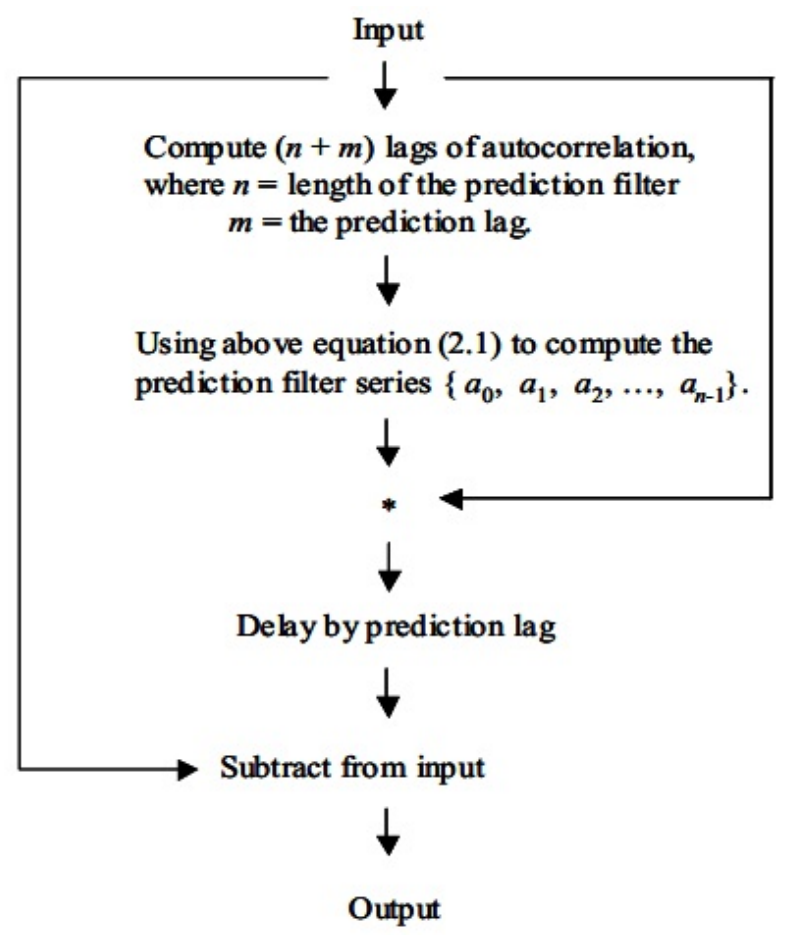

Figure 2.1: A flowchart for predictive deconvolution using prediction filters. (Yilmaz, 2001) chapter2/pd pd1 


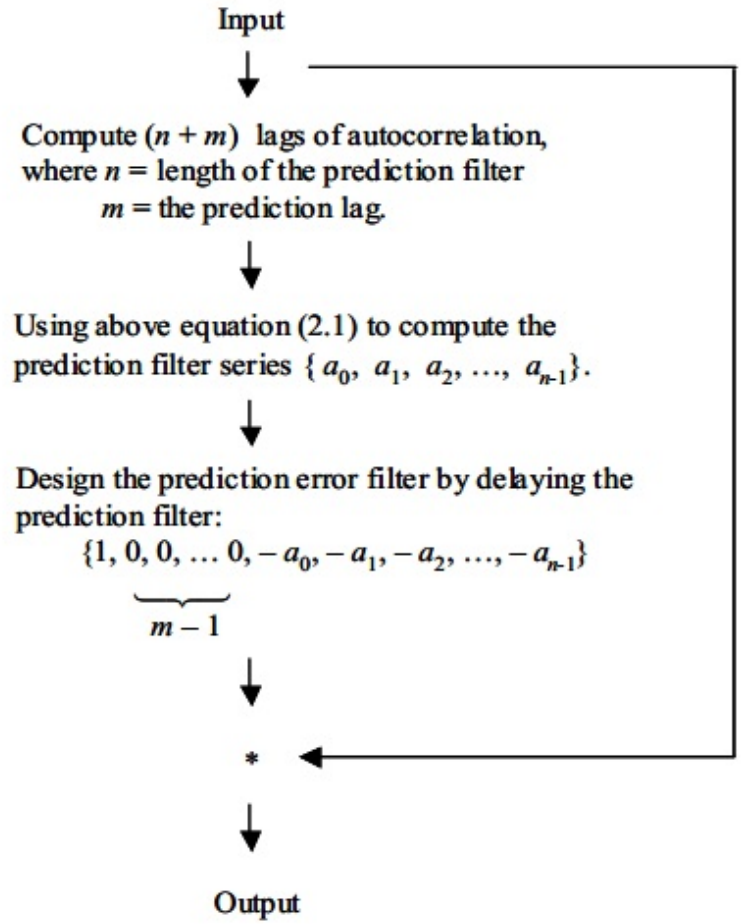

Figure 2.2: A flowchart for predictive deconvolution using prediction error filters. (Yilmaz, 2001) chapter $2 / \mathrm{pd} \mathrm{pd} 2$ 
If all assumptions about the data are met (perfectly periodic multiples, stationary source waveform, minimum phase wavelet etc.), then all multiples should be suppressed. However, in practice, the demultiple operation might get more complicated if any of the basic assumptions are violated (Backus, 1959).

\section{SURFACE RELATED MULTIPLE ELIMINATION (SRME)}

Surface related multiple elimination (SRME) (Verschuur et al., 1988; Dragoset, 1998) is a pre-stack demultiple procedure carried out in the space-frequency $(\mathbf{x}-\omega)$ domain that does not require any knowledge of the sub-surface and can handle data from laterally inhomogenous sub-surfaces. It requires knowledge of only the reflection characteristics of the surface and the source wavefield. Using the matrix notation in Berkhout (1982), the full pre-stack monochromatic seismic data without any multiples can be represented as :

$$
\mathbf{P}_{\mathbf{0}}^{-}\left(z_{0}\right)=\mathbf{Q}\left(z_{0}\right) \mathbf{S}^{+}\left(z_{0}\right)
$$

where,

$$
\mathbf{Q}\left(z_{0}\right)=\sum_{m=1}^{M} \mathbf{W}^{-}\left(z_{0}, z_{m}\right) \mathbf{R}^{+}\left(z_{m}\right) \mathbf{W}^{+}\left(z_{0}, z_{m}\right)+\mathbf{M}_{\text {int }}^{-}\left(z_{0}\right)
$$

$\mathbf{P}_{\mathbf{0}}^{-}\left(z_{0}\right)$ is the upgoing wavefield at the surface $z=z_{0}$ and $\mathbf{S}^{+}\left(z_{0}\right)$ is the downgoing sourcefield. The data matrix $\mathbf{P}_{\mathbf{0}}^{-}\left(z_{0}\right)$ contains shot record along columns and zero-offset data along diagonals. The 
right hand side of equation 2.3 contains reflections from $\mathbf{M}$ interfaces and internal multiples, $\mathbf{M}_{\mathbf{i n t}}^{-}\left(z_{0}\right) . \mathbf{W}^{+}\left(z_{m}, z_{0}\right)$ represents the wave propagation from $z_{0}$ to $z_{m}, \mathbf{R}^{+}\left(z_{m}\right)$ the reflection at the interface $z=z_{m}$ and $\mathbf{W}^{-}\left(z_{0}, z_{m}\right)$ the propagation of the upgoing wave from $z_{m}=z_{0} \cdot \mathbf{Q}\left(z_{0}\right)$, which essentially represents the impulse response of the earth with a reflection free surface, is used as input for further processing. In reality however, the surface reflects back all the upgoing wavefields. This downgoing wavefield now acts as a new illuminating sourcefield. Essentially, the expression for the total upgoing wavefield containing all multiples, $\mathbf{P}^{-}\left(z_{0}\right)$, can be expressed as:

$$
\mathbf{P}^{-}\left(z_{0}\right)=\mathbf{Q}\left(z_{0}\right)\left[\mathbf{S}^{+}\left(z_{0}\right)+\mathbf{R}^{-}\left(z_{0}\right) P^{-}\left(z_{0}\right)\right]
$$

where, $\mathbf{R}^{-}\left(z_{0}\right)$ is the reflectivity matrix for upgoing waves at the surface. Equation 2.4 can be tranformed to write it in an explicit manner:

$$
\mathbf{P}^{-}\left(z_{0}\right)=\left[\mathbf{I}-\mathbf{Q}\left(z_{0}\right) \mathbf{R}^{-}\left(z_{0}\right)\right]^{-1} \mathbf{Q}\left(z_{0}\right) \mathbf{S}^{+}\left(z_{0}\right)
$$

The first term in equation 2.5 that generates all surface related multiples can be expanded using a Taylor's series as:

$$
\mathbf{P}^{-}\left(z_{0}\right)=\left[\mathbf{I}+\mathbf{Q}\left(z_{0}\right) \mathbf{R}^{-}\left(z_{0}\right)+\mathbf{Q}\left(z_{0}\right) \mathbf{R}^{-}\left(z_{0}\right)^{2}+\ldots .\right] \mathbf{Q}\left(z_{0}\right) \mathbf{S}^{+}\left(z_{0}\right)
$$

Each term on the right hand side of equation 2.6 represents one order of multiples. In order to 
eliminate multiples, 2.6 has to be re-written in a manner to get an explicit expression for $\mathbf{Q}\left(z_{0}\right)$ (impulse-response of the earth with a reflection-free surface):

$$
\mathbf{Q}\left(z_{0}\right)=\mathbf{P}^{-}\left(z_{0}\right)\left[\mathbf{S}^{+}\left(z_{0}\right)+\mathbf{R}^{-}\left(z_{0}\right) \mathbf{P}^{-}\left(z_{0}\right)\right]^{-1}
$$

or,

$$
\mathbf{Q}\left(z_{0}\right)=\mathbf{P}^{-}\left(z_{0}\right) \mathbf{S}^{-}\left(z_{0}\right)\left[\mathbf{I}+\mathbf{R}^{-}\left(z_{0}\right) \mathbf{P}^{-}\left(z_{0}\right) \mathbf{S}^{-}\left(z_{0}\right)\right]^{-1}
$$

where, $\mathbf{P}^{-}\left(z_{0}\right) \mathbf{S}^{-}\left(z_{0}\right)$ represents the data deconvolved for the source wavefield. Expressing $\mathbf{P}^{-}\left(z_{0}\right) \mathbf{S}^{-}\left(z_{0}\right)$ as $\mathbf{P}^{\prime}\left(z_{0}\right)$ and expanding equation 2.8 using a Taylor's series:

$$
\mathbf{Q}\left(z_{0}\right)=\mathbf{P}^{\prime}\left(z_{0}\right)-\mathbf{P}^{\prime}\left(z_{0}\right) \mathbf{R}^{-}\left(z_{0}\right) \mathbf{P}^{\prime}\left(z_{0}\right)+\mathbf{P}^{\prime}\left(z_{0}\right)\left[\mathbf{R}^{-}\left(z_{0}\right) \mathbf{P}^{\prime}\left(z_{0}\right)\right]^{2}-\ldots . .
$$

Equation 2.9 demonstrates how multiples can be eliminated without having to do any full matrix inversion as in equations 2.7 and 2.8 . This method is always stable if a specific number of terms in the right hand side of equation 2.9 is taken into account. Since the recording time of seismic data is finite, the number of orders of multiples is always a limited number and only a limited number of terms in equation 2.9 can be taken into account.

One should note that this demultiple procedure does not require any information except the source wavefield and the reflectivity of the surface. Since in an acoustic case, the free-surface will be totally 
reflecting for all angles of incidence one can replace the reflectivity matrix $\mathbf{R}\left(z_{0}\right)$ by a single reflection co-efficient $r_{0}=1$.

For the elastic case, when there is mode conversion at interfaces and data is available as separate $\mathbf{P P}, \mathbf{P S}, \mathbf{S P}$ and $\mathbf{S S}$ waves (where $\mathbf{P S}$ represents received upgoing $\mathbf{P}$-wave caused by a pure S wave source etc.), the full reflectivity matrices: $R_{P P}^{-}, R_{P S}^{-}, R_{S P}^{-}$and $R_{S S}^{-}$, must be taken into account. Equation 2.9 can be re-written as:

$$
\begin{aligned}
\left(\begin{array}{ll}
Q_{P P} & Q_{P S} \\
Q_{S P} & Q_{S S}
\end{array}\right) & =\left(\begin{array}{ll}
P_{P P} & P_{P S} \\
P_{S P} & P_{S S}
\end{array}\right)-\left(\begin{array}{ll}
P_{P P} & P_{P S} \\
P_{S P} & P_{S S}
\end{array}\right) \cdot\left(\begin{array}{ll}
R_{P P} & R_{P S} \\
R_{S P} & R_{S S}
\end{array}\right) \cdot\left(\begin{array}{ll}
P_{P P} & P_{P S} \\
P_{S P} & P_{S S}
\end{array}\right) \\
& \left.+\left(\begin{array}{ll}
P_{P P} & P_{P S} \\
P_{S P} & P_{S S}
\end{array}\right) \cdot\left[\begin{array}{ll}
R_{P P} & R_{P S} \\
R_{S P} & R_{S S}
\end{array}\right) \cdot\left(\begin{array}{ll}
P_{P P} & P_{P S} \\
P_{S P} & P_{S S}
\end{array}\right)\right]^{2}-\ldots
\end{aligned}
$$

This concept has also been extended to 3D in the last decade vanDehem and Verschuur, 2002 Hokstad and Sollie, 2003).

\section{WAVE EQUATION APPROACH}

Suppression of water-layer multiples and peg-legs by wave-equation approach was suggested by Lokshtanov (2000). Pre-stack data (in any domain) is denoted by $\mathbf{D}$ and the receiver-side extrapolation of the input data through the water layer is denoted by the operator $\mathbf{P}_{\mathbf{g}}$. The operator $\mathbf{P}_{\mathbf{g}}$ incorporates the following: propagation of the recorded wavefield down to the sea floor, reflection from the sea floor multiplied by the reflection co-efficient at the free surface (surface between water and air) and the propagation of the wave up to the free surface. Such an extrapolation results in the primary water-bottom reflection being transferred to the first-order multiple, the first-order multiple being transferred to the second-order multiple and so on. Similarly, each primary reflection from below the 
water bottom gets transferred to the first-order receiver side peg-leg, each first-order receiver side peg-leg is transferred to the second-order receiver side peg-leg and so on. Therefore, the operator $\left(\mathbf{I}+\mathbf{P}_{\mathbf{g}}\right) \mathbf{D}$ applied to the data $\mathbf{D}$ removes all water bottom multiples and receiver side peg-legs. The result $\mathbf{F}_{\mathbf{1}}=\left(\mathbf{I}+\mathbf{P}_{\mathbf{g}}\right) \mathbf{D}$ removes all the "pure" water-layer multiples and receiver side multiples (Lokshtanov, 2000).

One can use the principle of reciprocity to perform a similar operation for the source side. If the positions of the sources and receivers are interchanged, the source-side peg-legs in the original configuration become the receiver-side peg-legs. If the source-side extrapolation operator is denoted by $\mathbf{P}_{\mathbf{s}}$, the operator $\left(\mathbf{I}+\mathbf{P}_{\mathbf{s}}\right)$ applied on the new data from the previous step, $\mathbf{F}_{\mathbf{1}}$ can get rid of the receiver-side peg-legs after interchange of the source and receivers.

It is important to note that the operator $\mathbf{F}_{\mathbf{1}}$ contains the primary reflection from the water bottom and the operator, $\mathbf{P}_{\mathbf{s}}$, applied to $\mathbf{F}_{\mathbf{1}}$ creates first order pure water layer multiples which are already removed from $\mathbf{F}_{\mathbf{1}}$. Therefore, to suppress all pure water layer multiples and peg-legs, we have to perform the following operation:

$$
\mathbf{F}=\left(\mathbf{I}+\mathbf{P}_{\mathbf{s}}\right)\left(\mathbf{I}+\mathbf{P}_{\mathbf{g}}\right) \mathbf{D}-\mathbf{P}_{\mathbf{s}} \mathbf{D}_{\mathbf{w}}
$$

This reflection can be easily separated from the rest of the data (noise) in the $\tau-\mathbf{p}$ domain. The above equation 2.11 can be re-written as: 


$$
\mathbf{F}=\mathbf{D}+\mathbf{D}_{\mathbf{g}}+\mathbf{D}_{\mathbf{s}}+\mathbf{D}_{\mathbf{s g}}
$$

where, $\mathbf{D}_{\mathbf{g}}=\mathbf{P}_{\mathrm{g}} \mathbf{D}, \mathbf{D}_{\mathbf{s}}=\mathbf{P}_{\mathbf{s}}\left(\mathbf{D}-\mathbf{D}_{\mathbf{w}}\right)$ and $\mathbf{D}_{\mathbf{s g}}=\mathbf{P}_{\mathbf{s}} \mathbf{P}_{\mathbf{g}} \mathbf{D}$.

The extrapolation operators $\mathbf{P}_{\mathbf{g}}$ and $\mathbf{P}_{\mathbf{s}}$ include the reflectivity of the water-bottom, which is not known in practice. Therefore, it is assumed that reflectivity of the water-bottom is unity for all angles of incidence and all reflection points in the sea-floor. Therefore, the extrapolation that predicts the kinematics of multiples properly, but not their amplitudes, must be scaled properly. For each p-trace, a scaled version of equation 2.12 to the $\tau-\mathbf{p}$ transformed gathers has the form:

$$
f(\tau)=d(\tau)+r_{g}(\tau) * d_{g}(\tau)+r_{s}(\tau) * d_{s}(\tau)+r_{s g}(\tau) * d_{s g}(\tau)
$$

where, $d(\tau), d_{g}(\tau), d_{s}(\tau)$ and $d_{s g}(\tau)$ are the $p$-traces for the input data, results from extrapolation to the receiver-side, source-side and extrapolation of receiver-side followed by source side respectively. The filters, $r_{g}(\tau), r_{s}(\tau)$ and $r_{s} g(\tau)$, estimated from the minimum energy criterion of $f$, account for angle dependent reflectivity from the water-bottom and small phase-shifts due to imperfect knowledge of water-bottom geometry. 


\section{F-K FILTERING}

If a wave is travelling with a velocity $c$, frequency $f$ and wavelength $\lambda$, then the speed of the wave is given by the relation:

$$
c=\lambda f
$$

We know that wave number $k$ is related to the wavelength by the relation:

$$
k=\frac{2 \pi}{\lambda}
$$

From 2.14 and 2.15 we can say:

$$
f=\frac{c}{2 \pi} k
$$

Thus, we see that velocity is a slope in the $f-k$ domain. This property is exploited to eliminate multiples in this domain. Shot gathers are converted to CMP gathers and then NMO correction is applied with a velocity that is somewhere in between that of primaries and multiples. Since, such an operation leads to the over correction of primary reflections and under correction of multiple reflections, the former and the latter have slopes with opposite signs. Then the gather is converted from $t-x$ to $f$ - $k$ domain. Therefore, in the $f$ - $k$ domain, primaries and multiples map onto different quadrants. Thereafter, the multiple energy is fanned out, and the remaining primaries are converted back to $t$ - $x$ domain using inverse Fourier transform.

The main steps involved in $f-k$ filtering are (Meek and Linvelle, 1993): 
1. Form supergathers to prevent aliasing as conversion from shot to CMP domain can give rise to trace spacing increasing.

2. Pick primary velocities and apply NMO correction with a velocity that is somewhere in between primaries and multiples, so as to, over-correct primaries and under-correct multiples.

3. Convert to $f$ - $k$ using Fourier Transform.

4. Suppress multiple energy in the $f$ - $k$ domain.

5. Revert back to $t-x$ domain to obtain data with multiples suppressed.

Figure 2.3 is a synthetic CMP gather (Haskell data) that was created by elastic modelling. Figure 2.3(a) is the original gather and Figure 2.3(b) is the gather after Normal Moveout (NMO) correction. Figure 2.4(a) and (b) show the gather with NMO correction with primary velocity and $70 \%$ of the primary velocity respectively. It can be seen that while primaries appear flat and multiples under-corrected in Figure 2.4(a), in Figure 2.4(b), multiples appear under-corrected while primaries are over-corrected.

Figures 2.5(a) and (b) show the real part of the data (2.4(b)) in the $f k$ domain before (Figure 2.5(a)) and after (Figure 2.5(b)) muting out multiples. Figure 2.6 shows the result for the demultipled gather. Since the NMO correction kills some information due to NMO stretch, for the sake of fairness, I compare the demultipled gather with the original gather with forward and inverse NMO applied (Figure 2.6). Figure 2.6(b) shows the demultipled gather and Figure 2.6(c) shows the subtracted multiples, i.e, the portion that has been filtered out. It is clear from this figure that some multiples that appear in Figure 2.6(a) appear suppressed in Figure 2.6(b). This is further 


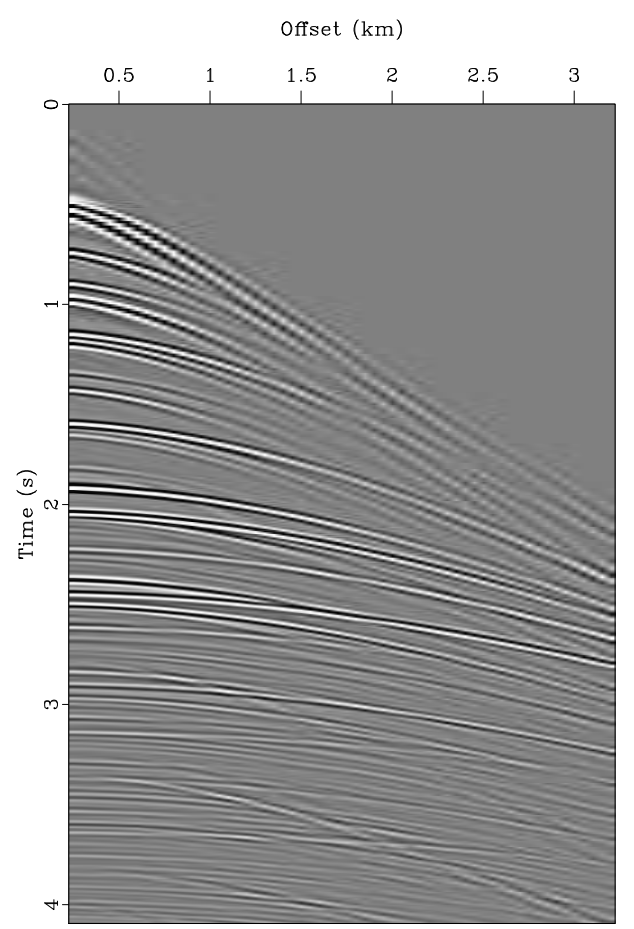

(a) Original gather

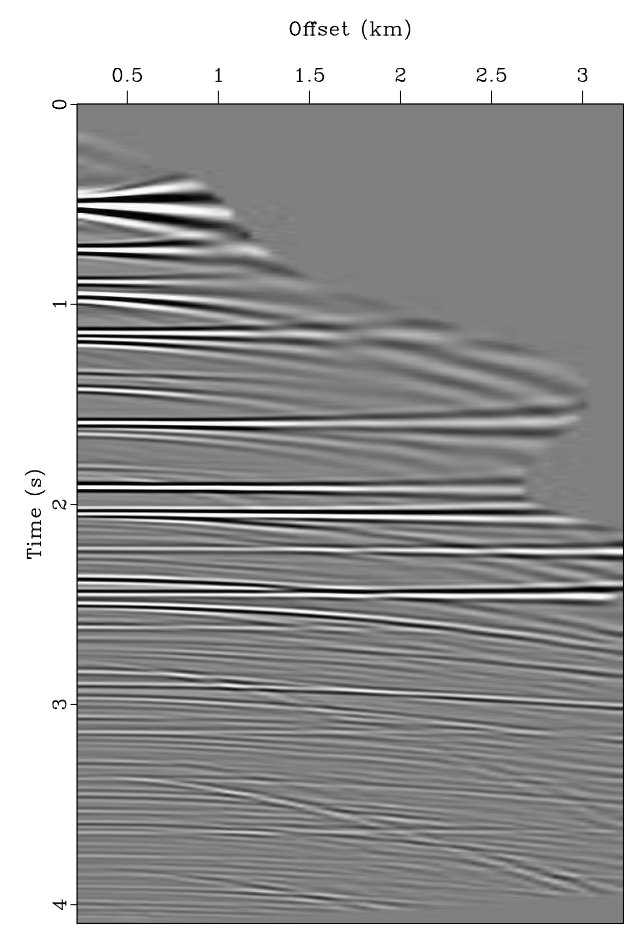

(b) NMO

Figure 2.3: (a) Original CMP gather, (b) NMO corrected gather with primary velocity. chapter2/haskellfk nmo 


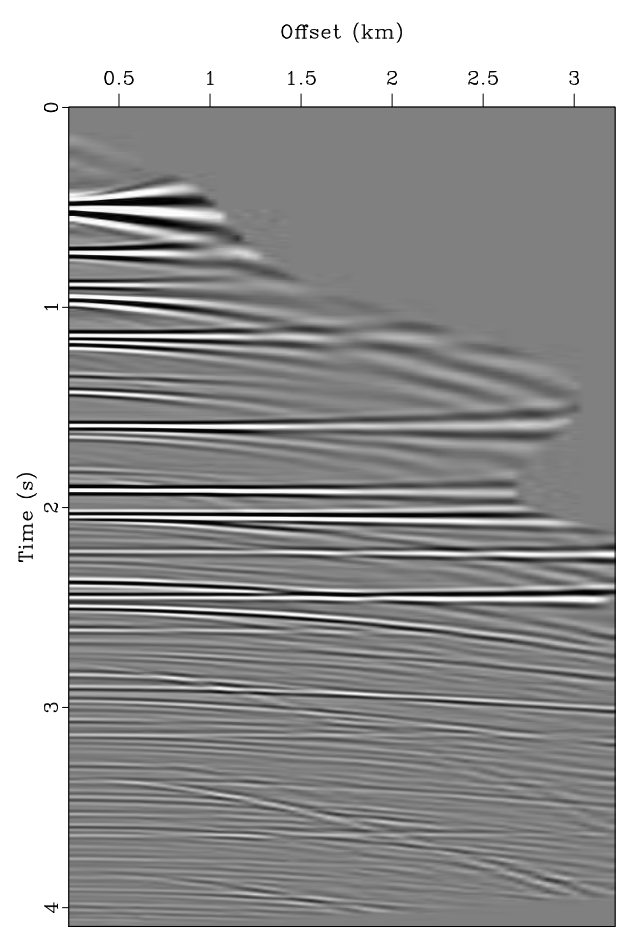

(a) NMO with primary velocity

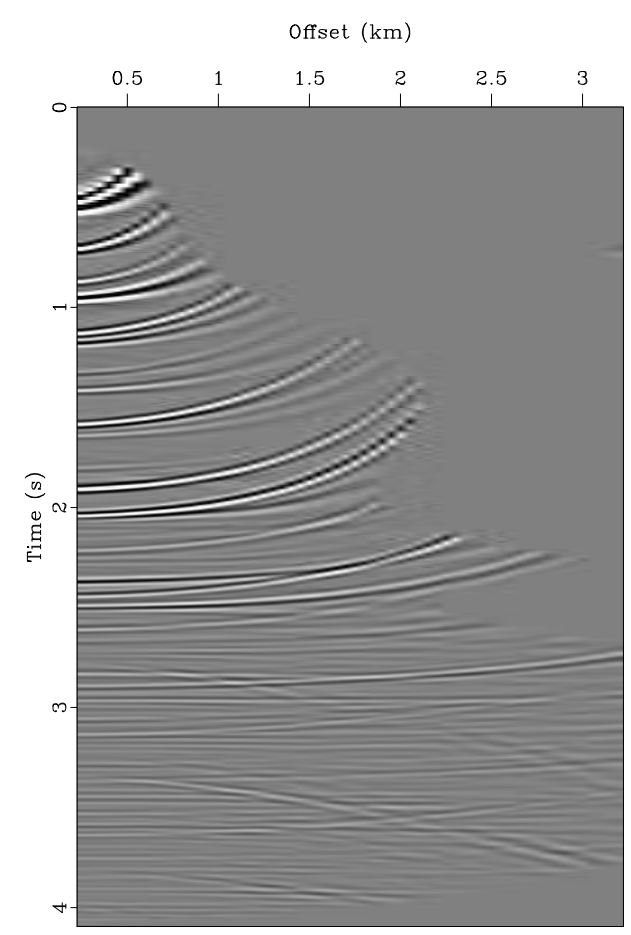

(b) NMO with $70 \%$ of primary velocity

Figure 2.4: (a) NMO corrected gather with primary velocity , (b) NMO corrected gather with $70 \%$ primary velocity. chapter2/haskellfk comparenmo 


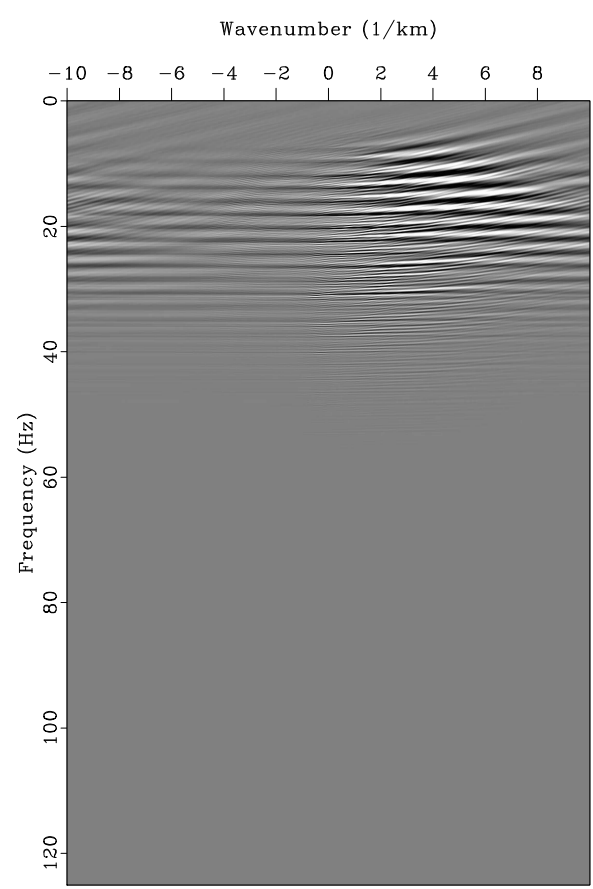

(a) Real part in fk domain

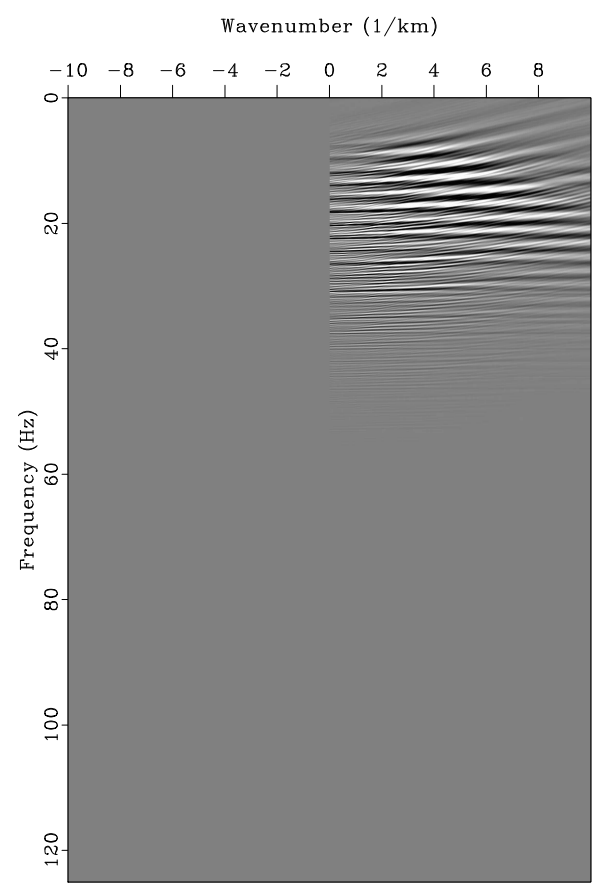

(b) Real part in fk domain after muting multiple

Figure 2.5: (a) Real part of the data in the $f k$ domain, (b) Real part of the data in the $f k$ domain after muting out the multiples. chapter2/haskellfk fkdata 


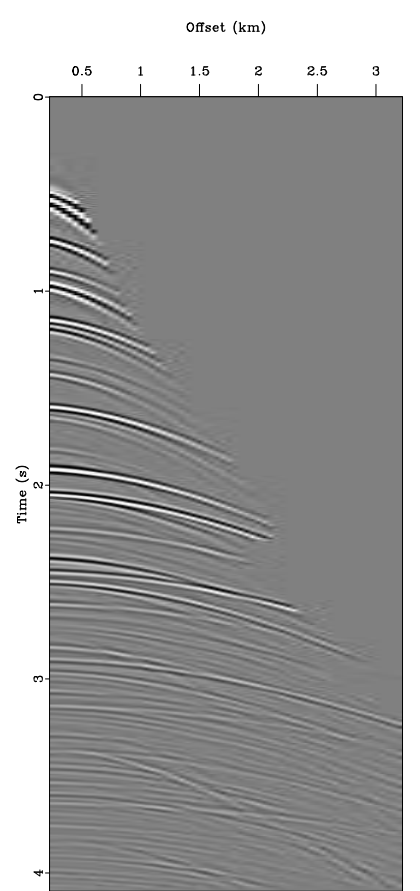

(a) Restored gather

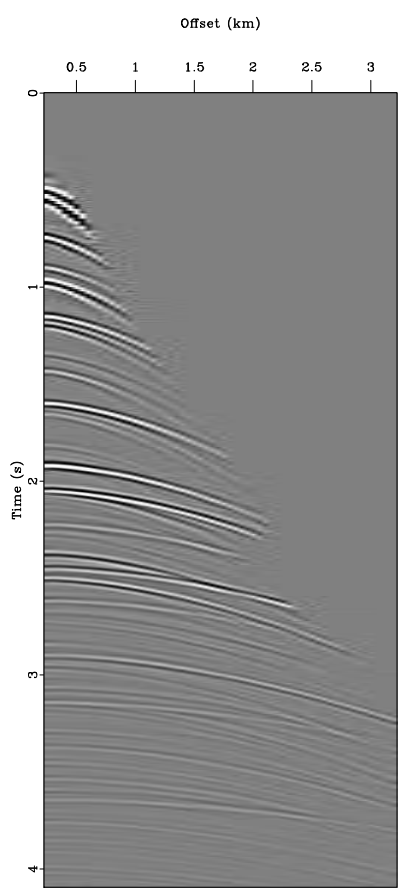

(b) Demultiple gather

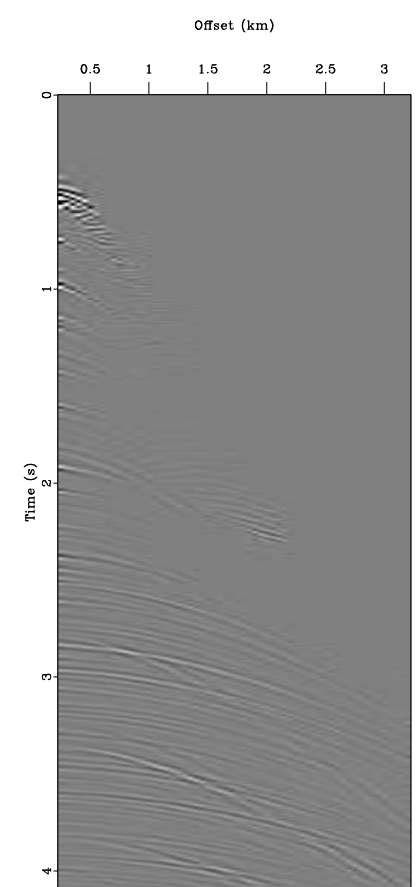

(c) Subtracted Multiples

Figure 2.6: (a) Restored gather after NMO and inverse NMO , (b) Demultipled gather using $f k$ filtering and (c) Subtracted multiples (difference between (a) and (b)). chapter2/haskellfk fkresult 


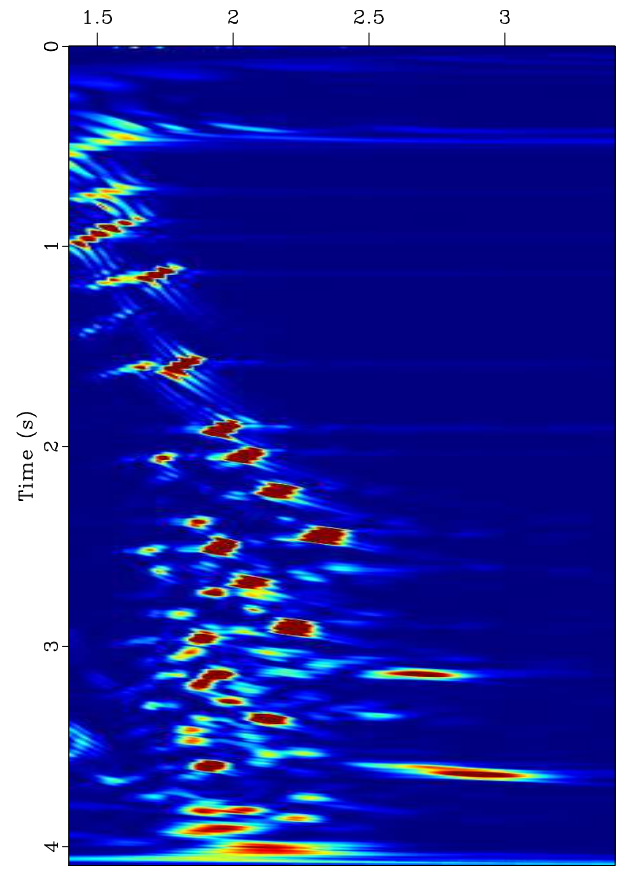

Velocity Scan

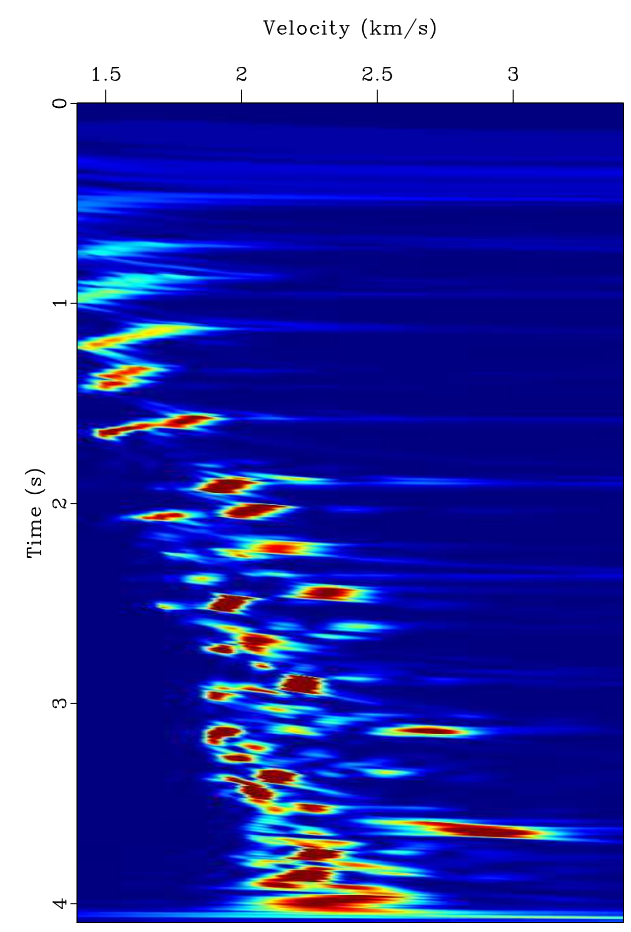

Velocity Scan

Figure 2.7: (a) Velocity scan before demultiple, (b) Velocity scan after demultiple. chapter2/haskellfk vscan 
reflected in the velocity scans before (Figure 2.7(a)) and after (Figure 2.7(b)) the demultiple operation. Some of the internal multiples that appear after 3s in Figure 2.7(a) appear suppressed in Figure 2.7(b), making primaries and higher order internal multiples more distinct.

\section{RADON TRANSFORMS}

The Radon transform methods converts data from the time-offset $(t-x)$ domain to the intercept time-slope $(\tau-p)$ domain, by either summing up the data along a line (linear Radon transform or slant stack) or hyperbolas (hyperbolic Radon transform) or parabolas (parabolic Radon transform). Thereafter, multiples are suppressed by applying an appropriate mute in the transformed $\tau-p$ domain and inverse transform is applied to obtain the data with multiples suppressed.

\section{Linear Radon transform or slant-stack}

Data is converted from the $t$ - $x$ domain to the $\tau$-p domain by summing along linear slopes. This involves two steps. First, a Linear Moveout (LMO) correction is applied to the data through a coordinate transformation (Claerbout, 1976, Stoffa et al., 1981):

$$
\tau=t-p x
$$

where $p$ is the horizontal slowness or ray parameter, $x$ is the offset, $t$ is the time in the $t-x$ section and $\tau$ is the intercept time. Thereafter, the data is summed along the offset axis to obtain the data in the transformed domain as per: 


$$
\phi(\tau, p)=\int d(\tau+p x, x) d x
$$

where, $d(\tau+p x, x)$ is data in the time-offset $(t-x)$ domain and $\phi(\tau, p)$ is data in the transformed slant stack $(\tau, p)$ domain. By applying LMO for different values of $p$ and then summing according to 2.18 we can obtain the entire slant stack that contains all the dips in the original offset data. One can go from the $(\tau, p)$ domain to the $(t, x)$ domain by applying the inverse transform that is given by the relation:

$$
d(t, x)=|\mathbf{D}|^{m} \int \hat{w} \phi(t-p x, p) d p
$$

where,

$$
\hat{w}=\frac{1}{(2 \pi)^{m}}
$$

$|\mathbf{D}|^{m}$ is a one-dimensional convolution operator with the spectrum $|w|^{(m / 2)}$ and $m$ is the dimensionality of $p$ and $x$ (usually 1 or 2 ). While applying the forward transform, a line maps onto a point and while applying the inverse transform, a point maps onto a straight line (Tatham, 1984). Straight line events such as refractions and direct arrivals therefore map onto points in the $(\tau, p)$ domain when one uses the linear Radon transform. Reflections, which are hyperbolic events, map onto ellipses when one uses the slant stack.

\section{Hyperbolic Radon transform}

Since seismic reflection events are mostly hyperbolic in nature, it is more appropriate to sum them along hyperbolic curves. This facilitates a better curve matching and is done as per:

$$
\phi(\tau, q)=\int d\left(\sqrt{\tau^{2}+q^{2} x^{2}}, x\right) d x
$$


where $d(t, x)$ is data in the $(t, x)$ domain and $\phi(\tau, q)$ is data in the transformed $(\tau, q)$ domain.

The inverse transform is given theoretically by the relation (Thorson and Claerbout, 1985):

$$
d(t, x) \approx|\mathbf{D}|^{m} \int \hat{w} \phi\left(\sqrt{t^{2}-q^{2} x^{2}}, q\right) d q
$$

where for $m=1$,

$$
\hat{w}=\frac{1}{\pi} \frac{q x}{t}
$$

$|\mathbf{D}|^{m}$ is a one-dimensional convolution operator with the spectrum $|w|$ and $m$ is the dimensionality of $q$ and $x$ (usually 1 or 2 ) and $q$ has the unit of $s / m$ or $s / f t$.

Equation 2.21 can be used to model hyperbolic events very well. Ideally, hyperbolas map onto a point in the transformed domain and a point in the transformed domain gets mapped onto a hyperbola upon application of the inverse transform. The cost of computing a least-squares inversion, via common methods like conjugate gradients is expensive for large data sets. The parabolic Radon transform, which is a good compromise to model NMO corrected hyperbolic events with sufficient accuracy and reasonable efficiency, is described in the next section.

\section{Parabolic Radon transform}

As explained in an earlier section, the hyperbolic Radon transform is computationally expensive. The hyperbolic reflections can be approximated by fitting them with parabolas after NMO correction. The forward transform is given by: 


$$
\phi(\tau, q)=\int d\left(\tau+q x^{2}, x\right) d x
$$

while the inverse transform is given by:

$$
d(t, x) \approx|\mathbf{D}|^{m} \int \hat{\omega} \phi\left(t-q x^{2}, q\right) d q
$$

where for $m=1$,

$$
\hat{\omega}=\frac{x}{\pi}
$$

$|\mathbf{D}|^{m}$ is a one-dimensional convolution operator with the spectrum $|w|$ and $m$ is the dimensionality of $q$ and $x$ (usually 1 or 2 ).

For all the Radon Transform methods, an appropriate section (that represents multiples) is fanned out and then the inverse transform is applied to get gathers with multiples suppressed.

For time invariant transforms like the slant-stack and the parabolic Radon transform, the cost of computing the least-squares inversion can be reduced by computing the transform and its inverse independently in the frequency domain (Trorey, 1961; Beylkin, 1987). Kostov (1990) further showed that for time invariant Radon transforms, the inversion operators have a Toeplitz structure that further speeds up the least squares inversion. The details of this is specified in the next section.

Figure 2.8(a) shows the data shown in Figure 2.3(b) in the $\tau$-p domain after application of parabolic Radon transform and Figure 2.8(b) shows the data in the $\tau-p$ domain after muting out the multiples. 
Figure 2.9 shows the result for demultiple of the gather in Figure 2.3 using Parabolic Radon Transform. Figure 2.9 (a) shows the gather after forward and inverse NMO for a like for like comparison. Figure 2.9 (b) shows the demultipled gather and Figure 2.9 (c) shows the subtracted multiples. It is clear from Figures 2.9(b) and (c) that some multiples have been removed. This is further reflected in Figures 2.10(a) and (b) that shows the velocity scans before and after demultiple. The water bottom multiple in Figure 2.10(a) at around 1s does not show up in Figure 2.10(b). Also, the internal multiples become more distinct in Figure 2.10(b) indicating suppression of more water bottom multiples.

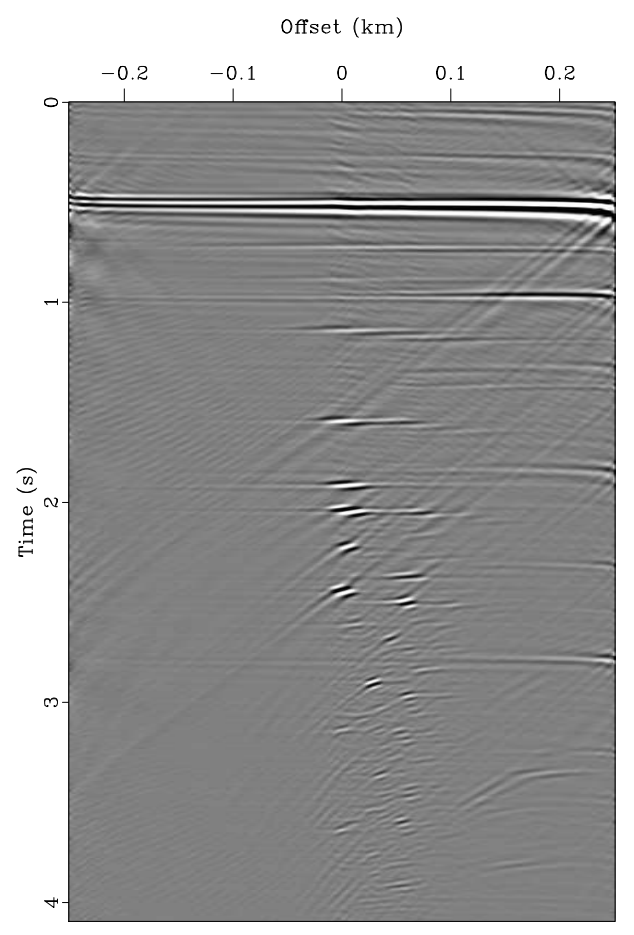

(a) Data in tau-p domain

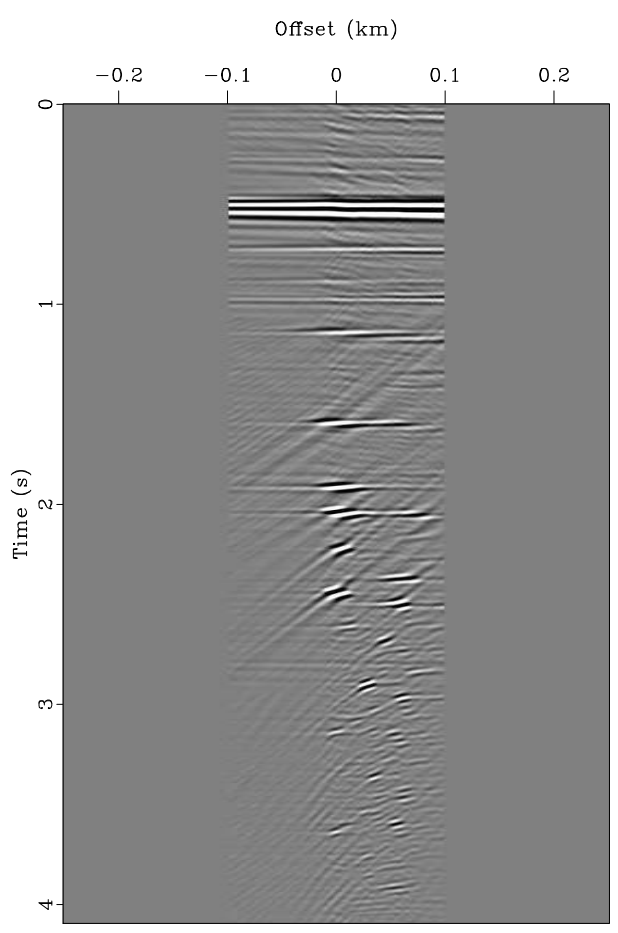

(b) Data in tau-p domain after muting

Figure 2.8: (a) Data in the $\tau$-p domain, (b) Data in the $\tau$-p domain after muting out the multiples. chapter2/haskellradon radondata 


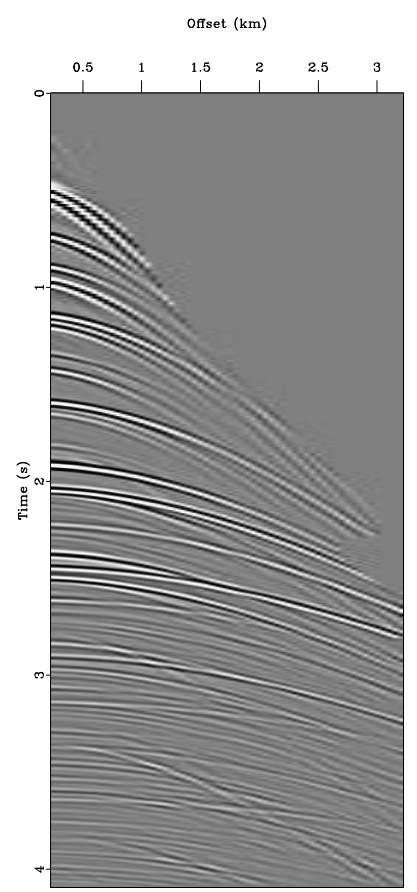

(a) Restored gather

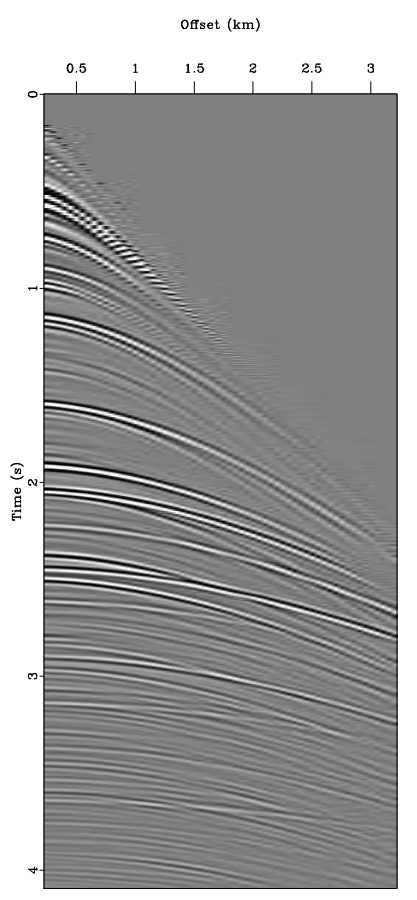

(b) Demultipled gather

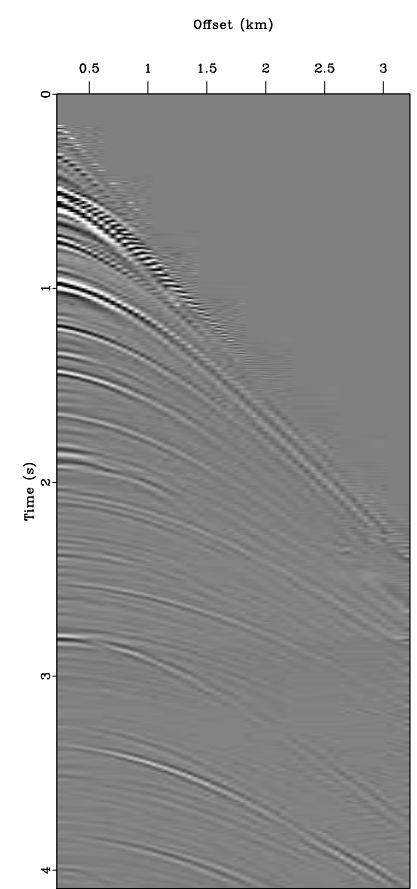

(c) Subtracted multiples

Figure 2.9: (a) Restored gather after NMO and inverse NMO , (b) Demultipled gather using Parabolic Radon and (c) Subtracted multiples (difference between (a) and (b)). chapter2/haskellradon demultipled 
Velocity $(\mathrm{km} / \mathrm{s})$

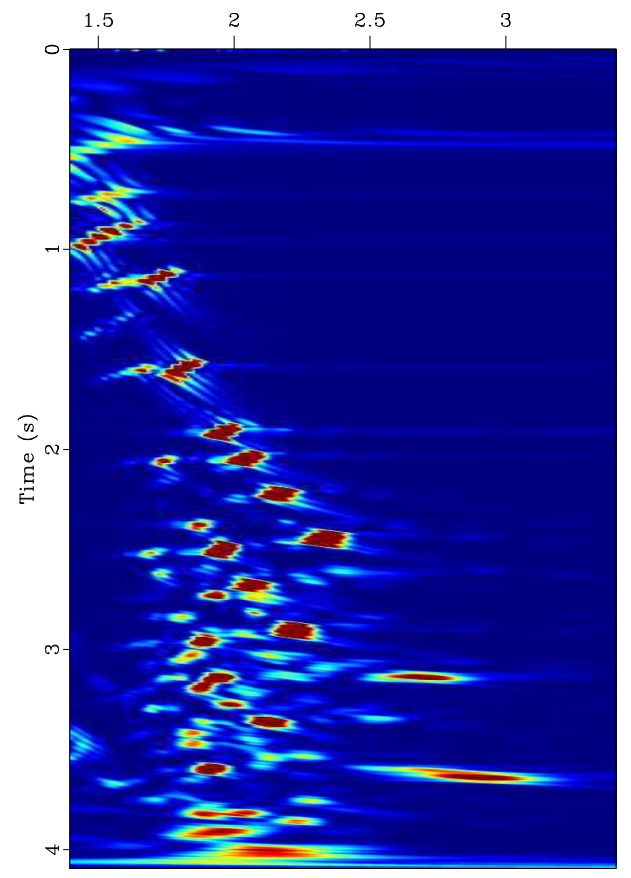

Velocity Scan

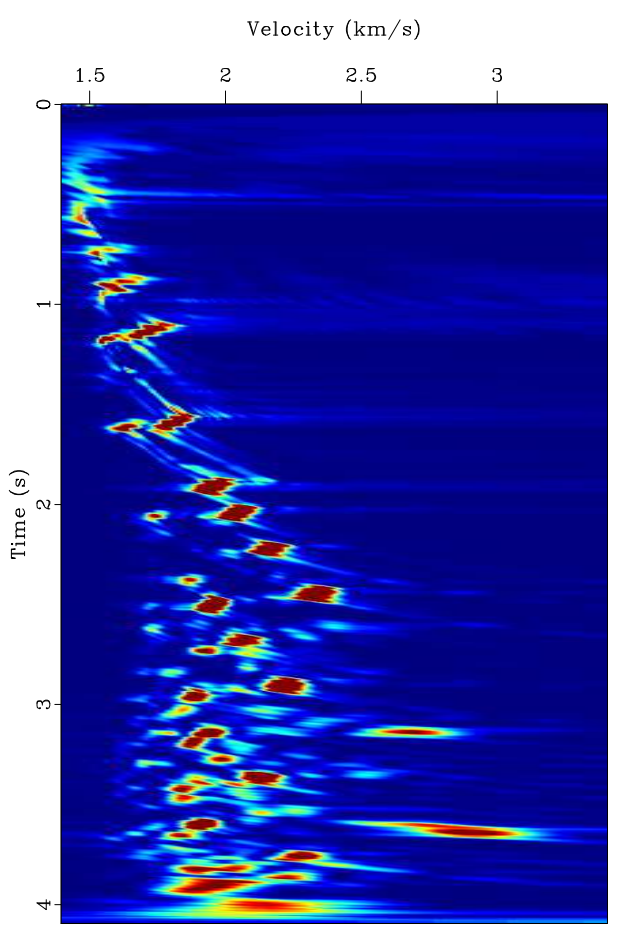

Velocity Scan

Figure 2.10: (a) Velocity scan before demultiple, (b) Velocity scan after demultiple. chapter2/haskellradon vscanr 


\section{Toeplitz structure for the inversion operator for time invariant Radon transforms}

The inverse and forward invariant Radon transforms can be written in the frequency domain as:

$$
\mathbf{M}: \hat{D}(\omega, x)=\int \phi(\omega, q) e^{-i \omega q x^{2}} d q
$$

and,

$$
\mathbf{M}^{\mathbf{T}}: \phi(\omega, q)=\int D(\omega, x) e^{i \omega q x^{2}} d x
$$

for the parabolic Radon transform and:

$$
\mathbf{M}: \hat{D}(\omega, x)=\int \phi(\omega, q) e^{-i \omega q x} d q
$$

and,

$$
\mathbf{M}^{T}: \phi(\omega, q)=\int D(\omega, x) e^{i \omega q x} d x ;
$$

for the slant-stack, where $\mathbf{M}^{\mathbf{T}}$ and $\mathbf{M}$ are the forward and inverse transform operators respectively. From equations 2.27 and 2.29 , for each frequency slice, we can write:

$$
F_{q}=\mathbf{M}_{(q, x)} D_{x}
$$

where $F_{q}$ has dimensions $n_{q}, D_{x}$ has dimensions $n_{x}$ and therefore the operator $\mathbf{M}$ has $n_{q}$ rows and $n_{x}$ columns. The least square inverse operator of the matrix $\mathbf{M}$ can be calculated as:

$$
F_{q}=\left(\mathbf{M}_{(q, x)}^{\mathbf{T}} \mathbf{M}_{(q, x)}\right)^{-1} \mathbf{M}_{(q, x)}^{\mathbf{T}} D_{x}
$$


The operator $\mathbf{M}_{(q, x)}^{\mathbf{T}} \mathbf{M}_{(q, x)}$ can be calculated as:

$$
\mathbf{M}_{(q, x)}^{\mathbf{T}} \mathbf{M}_{(q, x)}: \phi(\omega, q)=\iint\left(\phi\left(\omega, q_{0}\right) e^{-i \omega q_{0} x^{2}+i \omega q x^{2}} d q_{0} d x\right.
$$

or,

$$
\mathbf{M}_{(q, x)}^{\mathbf{T}} \mathbf{M}_{(q, x)}: \phi(\omega, q)=\iint\left(\phi\left(\omega, q_{0}\right) e^{-i \omega\left(q_{0}-q\right) x^{2}} d q_{0} d x ;\right.
$$

Changing the order of integration and denoting the inner matrix as:

$$
A\left(q, q_{0}\right)=\int e^{-i \omega\left(q_{0}-q\right) x^{2}} d x
$$

We can see that the matrix $A\left(q, q_{0}\right)$ is Toeplitz as the elements are constant along diagonals. In the case of slant-stack, the equivalent for equation 2.34 is:

$$
\mathbf{M}_{(q, x)}^{\mathbf{T}} \mathbf{M}_{(q, x)}: \phi(\omega, q)=\iint\left(\phi\left(\omega, q_{0}\right) e^{-i \omega\left(q_{0}-q\right) x} d q_{0} d x\right.
$$

and matrix $A\left(q, q_{0}\right)$ becomes:

$$
A\left(q, q_{0}\right)=\int e^{-i \omega\left(q_{0}-q\right) x} d x
$$

Once again the matrix $A\left(q, q_{0}\right)$ is Toeplitz. 


\section{Chapter 3}

\section{Multiple suppression in the $t-x-p$ domain*}

As described in the previous chapter, one family of velocity filters for demultiple operations involves using Radon transforms (Hampson, 1986, 1987, Foster and Mosher, 1992, Sacchi and Porsani, 1999 Herrmann et al., 2000, Abbad et al., 2011). Some of the Radon transform methods traditionally face challenges when the water column is shallow. Additionally, the hyperbolic Radon Transform (Foster and Mosher, 1992) can be computationally expensive. The demultiple technique presented in this chapter aims at tackling some of these limitations. The demultiple technique involves essentially two steps: the decomposition part and the suppression part. Both these steps are described in the sections below.

In this chapter, I discuss a novel multiple suppression technique in the $t-x-p$ domain, where $p$ is the local slope of seismic events, and discuss the merits, demerits and scope of this approach. In this approach, Common Mid-Point (CMP) gathers are taken and transformed from the original $t-x$ space to the extended $t-x-p$ space and eventually to the $t_{0}-x-p$ space, where $t_{0}$ is the zero offset traveltime. Thereafter, velocities are intrinsically calculated for each point in the extended domain in terms of $t_{0}, x$ and $p$ using analytical relations described in a later section and then Gaussian tapering filters are formed using difference of the powers of the intrinsically calculated velocities and the picked

\footnotetext{
*Parts of this chapter have been previously published in (Ghosh and Fomel, 2012). Fomel supervised this project.
} 
primary velocities. Multiplication of the gather in the extended space with such filters and stacking along the $p$ axis produces a gather with multiples suppressed.

\section{THEORY}

\section{Slope decomposition and the $t-x-p$ domain}

Slope decomposition transforms seismic data from the original $t-x$ domain to the extended $t-x-p$ domain, where $p$ is the local slopes of seismic events. A summation over $p$ restores the original gather with all amplitudes preserved. The idea of such a decompostion was first introduced by Ottolini (1983a) and has been acomplished previously using the local-slant stack (windowed Radon) transforms (Ventosa et al. 2012). However, the fundamental slope-distance uncertainty makes it difficult to control the resolution of local slant stacks and to select appropriate window parameters.

Ghosh and Fomel (2012) proposed a different slope-decomposition algorithm based on the time-frequency decomposition developed by Liu and Fomel (2013). There are three steps in the decomposition:

1. Transformation from $t-x$ to $f-x$ domain by applying fast Fourier transform (FFT) along the time axis.

2. Applying regularized non-stationary regression (Fomel, 2009) to transform from $f$ - $x$ to $f-x-k$ or, equivalently, $f-x-p$ domain ,to each frequency slice. Regularized non-stationary regression (RNR) amounts to finding complex coefficients $a_{n}(f, x)$ in the decomposition

$$
d(f, x)=\sum_{n=1}^{N_{p}} d_{n}(f, x),
$$


where $d(f, x)$ is the data in $f-x$ domain,

$$
d_{n}(f, x)=a_{n}(f, x) e^{i 2 \pi f x p_{n}}
$$

and $p_{n}$ are sampled slopes. The variability and resolution of $a_{n}$ coefficients are controlled by shaping regularization (Fomel, 2007a). This step is analogous to the time-frequency decomposition by Liu and Fomel (2013). Decomposition from $t-x$ to $x-f-k$ domain using the technique described in Liu and Fomel (2013) is described in a later section.

3. Transformation from $f-x-p$ to $t-x-p$ domain by applying inverse FFT on the time axis.

The cost of decomposition is $O\left(N_{t} N_{x} N_{p} N_{i t e r}\right)$, where $N_{t}, N_{x}$ and $N_{p}$ are data dimensions in the $t-x-p$ domain. $N_{t}$ is the number of points in the time direction, $N_{x}$ is the number of points in the offset direction and $N_{p}$ is the number of points in the slope direction after decompostion of the gather. $N_{\text {iter }}$ is the number of iterations in least-squares inversion by shaping regularization (typically around 10). In comparison, the cost of the least-squares in version of the parabolic Radon transform after Levison's recursion is $O\left(N_{t}\left[N_{p}^{2}+N_{p} N_{x}\right]\right)$, where $N_{t}$ is the number of points in the time direction, $N_{x}$ is the number of points in the offset direction and $N_{p}$ is the number of points in the slope direction.

The decompostion from $f$ - $x$ to $f-x-p$ in Step 2 can be parallelized along the frequency $f$ axis or along the gather number axis while computations in Steps 1 and 3 that deal with the forward and inverse Fourier transforms respectively can be parallelized in $x$ or $p$ or gather number axis. For better load balancing, it is advantageous to parallelize data along the gather number axis.

Figures 3.1 and 3.2 illustrate different steps of the algorithm: Figure 3.1(a) shows a synthetic CMP 
gather in the $t$ - $x$ domain before any transformation and Figure 3.1(b) shows the gather after transforming it from $t$ - $x$ to $f$ - $x$ domain (Step 1 ). Figure $3.2(\mathrm{a})$ shows the real part of the complex-valued gather in the extended $f-x-p$ domain (Step 2). Figure 3.2(b) shows the final $t-x-p$ decomposition after Step 3. If one stacks the data shown in Figure 3.2(b) along the $p$ axis, then the original gather shown in Figure 3.1(a) is restored.

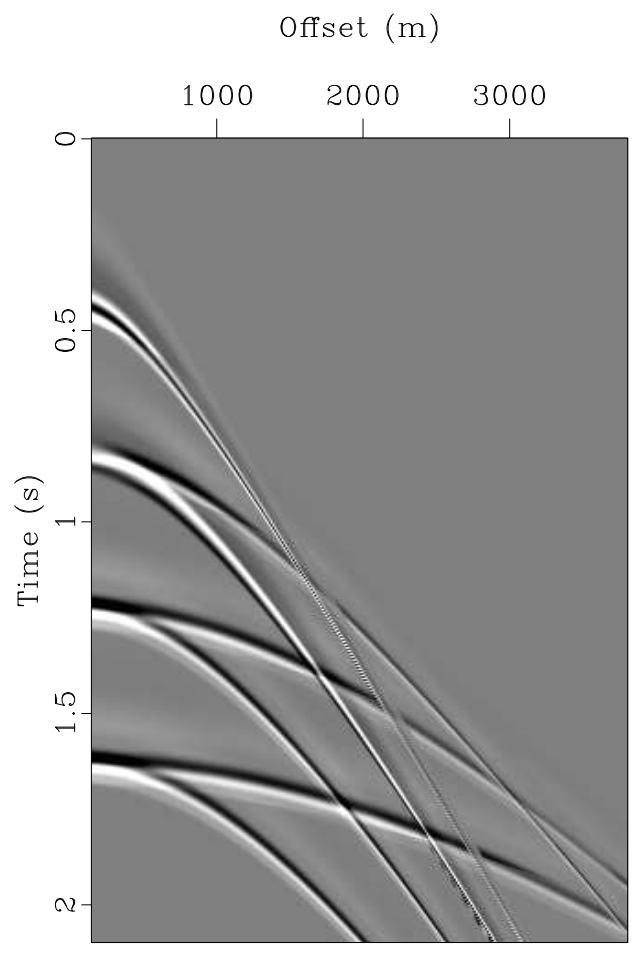

(a)

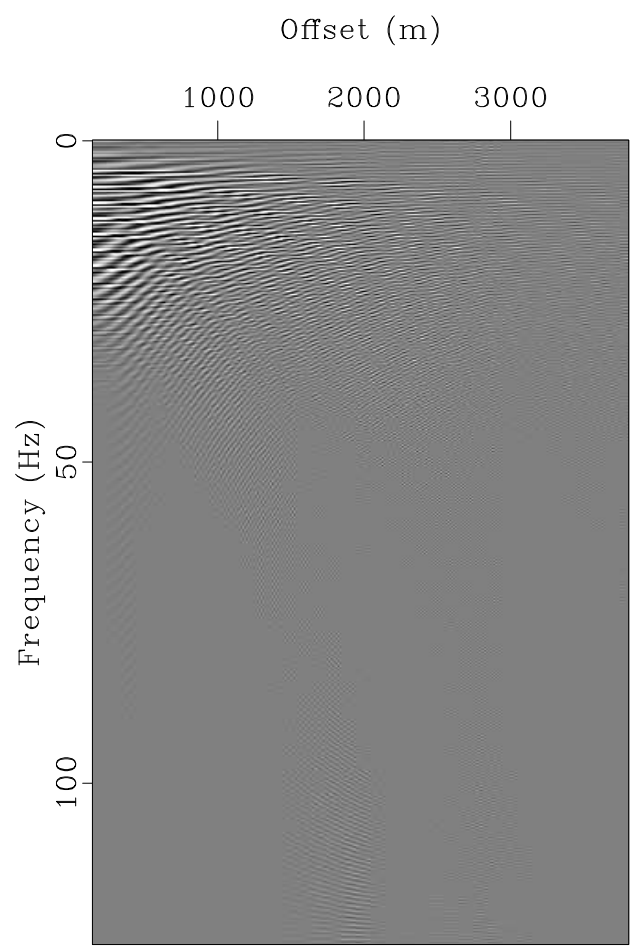

(b)

Figure 3.1: Synthetic CMP gather in $t$ - $x$ domain (a) and $f$ - $x$ domain (b,real part). chapter3/synthetic fx 


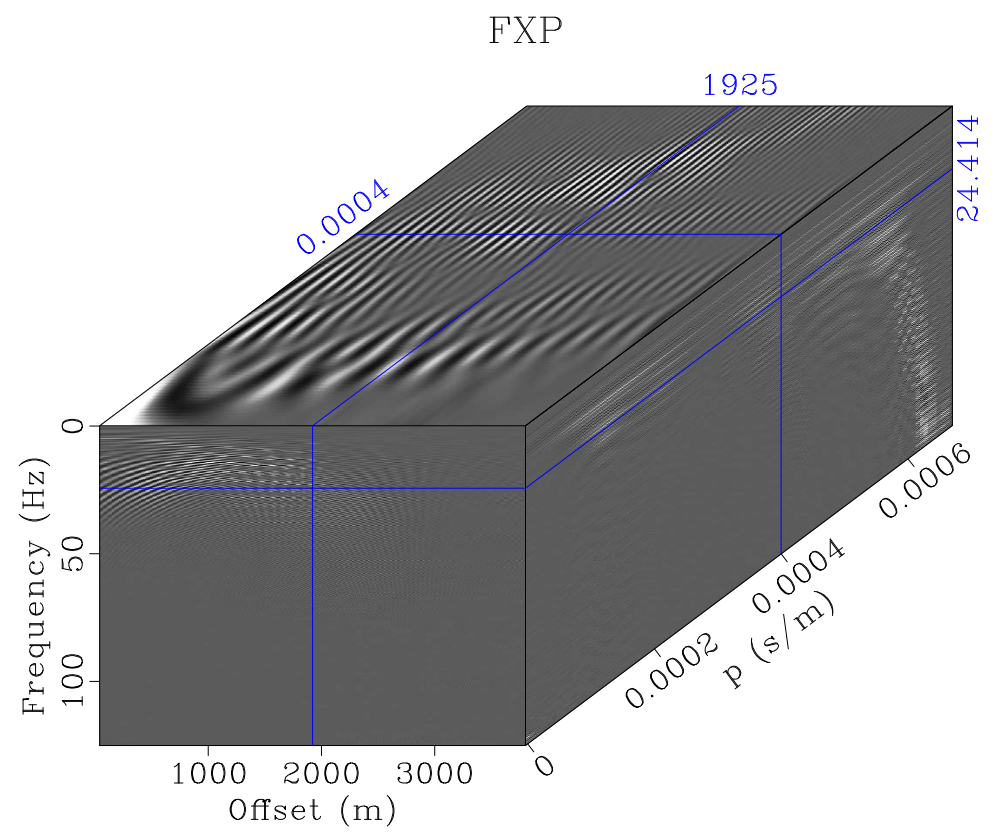

(a)

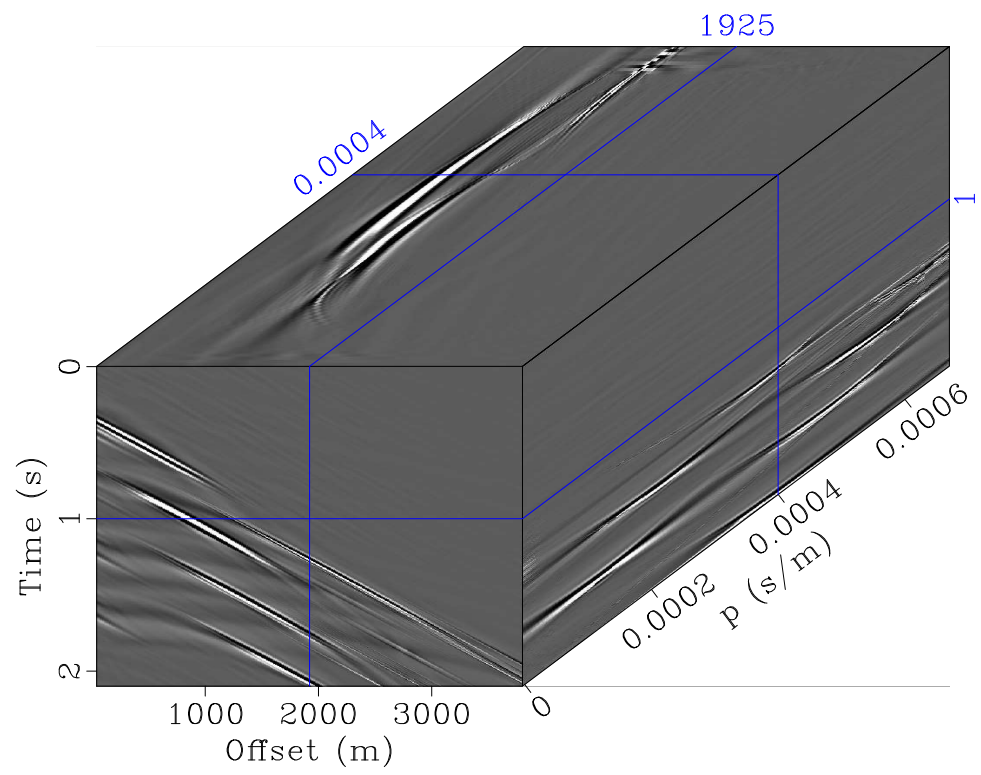

(b)

Figure 3.2: The CMP gather in the extended $f-x-p$ domain (a, real part) and $t-x-p$ domain (b). Stacking over $p$ restores the gather in Figure 3.1 a. chapter3/synthetic tx-fxp,txps 


\section{Decomposition from $t-x$ to $f-x-k$}

Liu et al. (2009, 2011) introduced a new method for time-varying frequency characterization of non-stationary seismic signals that was based on regularized least-squares inversion. Liu and Fomel (2013) expanded the method of Liu et al. (2011) by designing an invertible non-stationary time-frequency decomposition and its extensions: Local time-frequency-wavenumber (LTFK) and local space-frequency-wavenumber (LXFK) decompositions.

\section{Local time frequency LTF decomposition}

Fourier series is defined as an expansion of a function in terms of a sum of sines and cosines. The concept of Fourier series can be extended to complex co-efficients as per:

$$
f(x)=\sum_{-\infty}^{\infty} a_{n} \phi_{n}(x),
$$

where, $a_{n}$ are the Fourier co-efficients and:

$$
\phi_{n}(x)=e^{(2 \pi n x / L)},
$$

Nonstationary regression allows the coefficients of $a_{n}$ to change with $x . a_{n}(x)$ can be solved by solving the ill-posed (more unknown variables than constraints) least-squares minimization problem:

$$
\min _{a_{n}}\left\|f(x)-\sum_{n} a_{n}(x) \phi_{n}(x)\right\|_{2}^{2},
$$

Liu and Fomel (2013) includes additional constraints to limit the variability of the estimated coefficients (Fomel, 2009). Tikhonov's regularization (Tikhonov, 1962) can modify the objective function to: 


$$
\tilde{a}_{n}(x)=\arg \min _{a_{n}}\left\|f(x)-\sum_{n} a_{n}(x) \phi_{n}(x)\right\|_{2}^{2}+\epsilon^{2} \sum_{n}\left\|\mathbf{D}\left[a_{n}(x)\right]\right\|_{2}^{2},
$$

where, $\mathbf{D}$ is the regularization operator and $\in$ is a scaling parameter.

Liu and Fomel (2013) use shaping regularization (Fomel, 2007a) instead of Tikhonov's regularization. The absolute value of the co-efficients $\left|a_{n}(x)\right|$ provides a time-frequency representation and equation 3.2 provides the inverse calculation.

\section{Local $t-f-k($ LTFK) and local $x-f-k$ (LXFK) decompositions}

Based on the S transform, Askari and Siahkoohi (2008) proposed $t-f-k$ and $x-f-k$ transforms. Liu and Fomel (2013) defined new analogous LTFK and LXFK decompositions by using the LTF decomposition, that could be used to design time-varying and space-varying FK filters. The key steps in their algorithm are shown in Figures 3.3 and 3.4 


\section{Forward LTFK decomposition}

\begin{tabular}{|c|c|c|c|c|}
\hline Data & LTFD along $t$ axIS & $t-f-x$ & FFI along $x$ axis & $t-f-k$ \\
\hline$d(t, x)$ & ILTFD along $t$ axis & domain & IFFT along $x$ axis & domain \\
\hline
\end{tabular}

Figure 3.3: Schematic illustration of LTFK deomposition. chapter3/synthetic LTFK 
Forward LXFK decomposition

\begin{tabular}{|c|c|c|c|c|}
\hline $\begin{array}{l}\text { Data } \\
d(t, x)\end{array}$ & IFD along $x$ axis & $\begin{array}{c}t-x-k \\
\text { domain }\end{array}$ & $\frac{\text { FFT along } t \text { axis }}{\text { IFFT }}$ & $\begin{array}{c}x-f-k \\
\text { domain }\end{array}$ \\
\hline
\end{tabular}

Inverse LXFK decomposition

Figure 3.4: Schematic illustration of LXFK deomposition. chapter3/synthetic LXFK 


\section{Multiple suppression in the $t-x-p$ domain}

The hyberbolic traveltime equation is conventionally written as (Dix, 1955):

$$
t^{2}=t_{0}^{2}+\frac{x^{2}}{V^{2}}
$$

where $t_{0}$ is the zero-offset traveltime, $x$ is the offset and $V$ is the normal moveout velocity. If we take a derivative for equation 3.6 with respect to offset, $x$, we get the following:

$$
t \frac{d t}{d x}=\frac{x}{V^{2}}
$$

or

$$
p=\frac{d t}{d x}=\frac{x}{t V^{2}}
$$

where $p$ is the slope or horizontal slowness. From equation 3.8, we can express the moveout velocity in terms of slope $(p)$, offset $(x)$ and two-way traveltime $(t)$. Slope is a data attribute that can be estimated using various methods like local slant-stacks (Ottolini, 1983b), plane wave destruction (Fomel, 2002), structure-tensors (Luo et al., 2006) etc. Such an expression would enable us to write the traveltime equation without velocity in terms of slopes as (Ottolini, 1983b; Fomel, 2007b):

$$
t^{2}=t_{0}^{2}+t p x
$$

This equation is basically a quadratic in $t$ that can be solved for $t$ as a function of $t_{0}, p$, and $x$ using the $a-b-c$ formula. We can eliminate one of the solutions obtained by solving the quadratic using the boundary condition $t=t_{0}$ at $x=0$ and solve for $t$ as:

$$
t=\frac{p x+\sqrt{p^{2} x^{2}+4 t_{0}^{2}}}{2}
$$


Equation 3.8 gives us $V$ in terms of $p, t$ and $x$ while equation 3.10 gives us $t$ is terms of $t_{0}, p$, and $x$. Combining equations 3.8 and 3.10 , we can solve for $V$ in terms of $t_{0}, p$, and $x$ as:

$$
V^{2}=\frac{2 x}{p^{2} x+p \sqrt{p^{2} x^{2}+4 t_{0}^{2}}} .
$$

Equation 3.11 allows us to calculate velocity, $V$ at each point in the extended $t_{0}-x-p$ domain. After the velocity at each point in the extended domain is calculated, we use the picked primary velocities, $V_{\text {pick }}$ to form Gaussian filters of the form:

$$
F\left(t_{0}, x, p\right)=e^{-q f\left(t_{0}, x, p\right)^{2}} .
$$

where, $q$ is a large number and $f\left(t_{0}, x, p\right)$ is a function given by:

$$
f\left(t_{0}, x, p\right)=\left(\frac{1}{V_{\text {pick }}^{2 n}}-\frac{1}{V_{\text {cal }}^{2 n}}\right)^{2 m}
$$

where, $V_{\text {pick }}$ is the picked primary velocity, $V_{\text {cal }}$ is the calculated velocity in the extended $t_{0}-x-p$ domain as per equation 3.11 and $(m, n) \in N$.

We use the inverse of velocity so that we can deal with small numbers and control the magnitude of the filter co-efficient by just varying $q$ in equation 3.12 and use $(m, n) \in N$ to ensure that the exponential in equation 3.12 is always raised to a negative power to make $F\left(t_{0}, x, p\right) \in[0,1]$. The coefficient $q$ is adjusted in a manner that $F\left(t_{0}, x, p\right)$ goes to unity for primaries and zero for any events that are not primaries (including multiples). 


\begin{abstract}
ALGORITHM
The steps involved in the $t-x-p$ algorithm can be summarized as follows:
\end{abstract}

1. Take the $t-x$ gather and decompose it into the $t-x-p$ domain.

2. Convert each point in the $t-x-p$ cube to $t_{0}-x-p$ using equation 3.9 .

3. Pick the primary velocity trend $V\left(t_{0}\right)$, form the filter $F\left(V\left(t_{0}\right), t_{0}, x, p\right)$ for each point in the $t_{0}-x-p$ cube, and multiply the filter with the data.

4. Revert back to $t-x-p$ cube using equation 3.9 or, alternatively, stack over $x$ to produce a stacked $t_{0}-p$ section.

5. Stack along $p$ axis to obtain gather with multiples suppressed.

The multiple suppression technique can be parallelized very easily along the gather axis with minimal load unbalance (as there is equal number of computations to be performed for each gather) and a good degree of strong scalabity (speed-up increases with increase in number of processors).

\title{
SYNTHETIC DATA EXAMPLE
}

The algorithm described in the previous section is demonstrated with a synthetic CMP gather (Haskell data) shown in Figure 2.3 that was created by elastic modelling. The water bottom traveltime is around 0.5s (Figure 2.3(a) and the data contains water bottom multiples of several orders,

along with other kind of multiples (peg-leg, intra and interbed etc.). This is shown in Figure 2.3(b) where a Normal Moveout (NMO) correction has been applied to the CMP gather in Figure 2.3(a). The water-bottom multiples that start from $1.0 \mathrm{~s}, 1.5 \mathrm{~s}, 2.0 \mathrm{~s}$ and so on appear under-corrected. Other 
kind of multiples also appear under-corrected.

Figure 3.5 shows the gather in Figure 2.3(a), in the extended $t-x-p$ domain. Stacking along the $p$ axis restores the original gather. Figure 3.6(a) shows the reconstructed gather and Figure 3.6(b) shows the difference between the original gather (Figure 2.3(a)) and the reconstructed gather (Figure 3.6(a)). It can be seen that the reconstruction is almost perfect (except some negligible rounding off errors etc.).

Figure 3.7 shows the result of demultiple in the $t-x-p$ domain. Figure 3.7(a) shows the gather after demultiple and 3.7(b) shows the difference between the original gather (Figure 2.3(a)) and the demultipled gather. I have made an effort to preserve as much of the primaries as possible while killing a reasonable amount of multiples. A close look at Figure 3.7(a) reveals that most of the water bottom multiples and other kinds of multiples have been suppressed. Furthermore, the difference section (Figure 3.7(b)) reveals that most of the multiples have been suppressed, while the leakage of primary reflections is negligible. This is further illustrated in Figure 3.8 that compares the NMO corrected gathers before (Figure $3.8(\mathrm{a})$ ) and after (Figure $3.8(\mathrm{~b})$ ) demultiple. It can be seen that most of the multiples have been suppressed, without any significant loss of primary reflections. Another important point to note is that a couple of internal multiples at around 2.3 and 2.5 seconds respectively are not well suppressed. This is because the velocity (or moveout) difference between these multiples and the primaries that originate from the same $t_{0}$ is small and it is not possible to kill an appreciable amount of multiples without killing significant amount of primaries. This is further reflected in Figure 3.9 that shows the velocity scans for the gather before (Figure 3.9 (a)) and after (Figure 3.9 (b)) demultiple. It is clear that some of the multiples are well suppressed and 
the higher order internal multiples become more distinct after the demultiple operation.

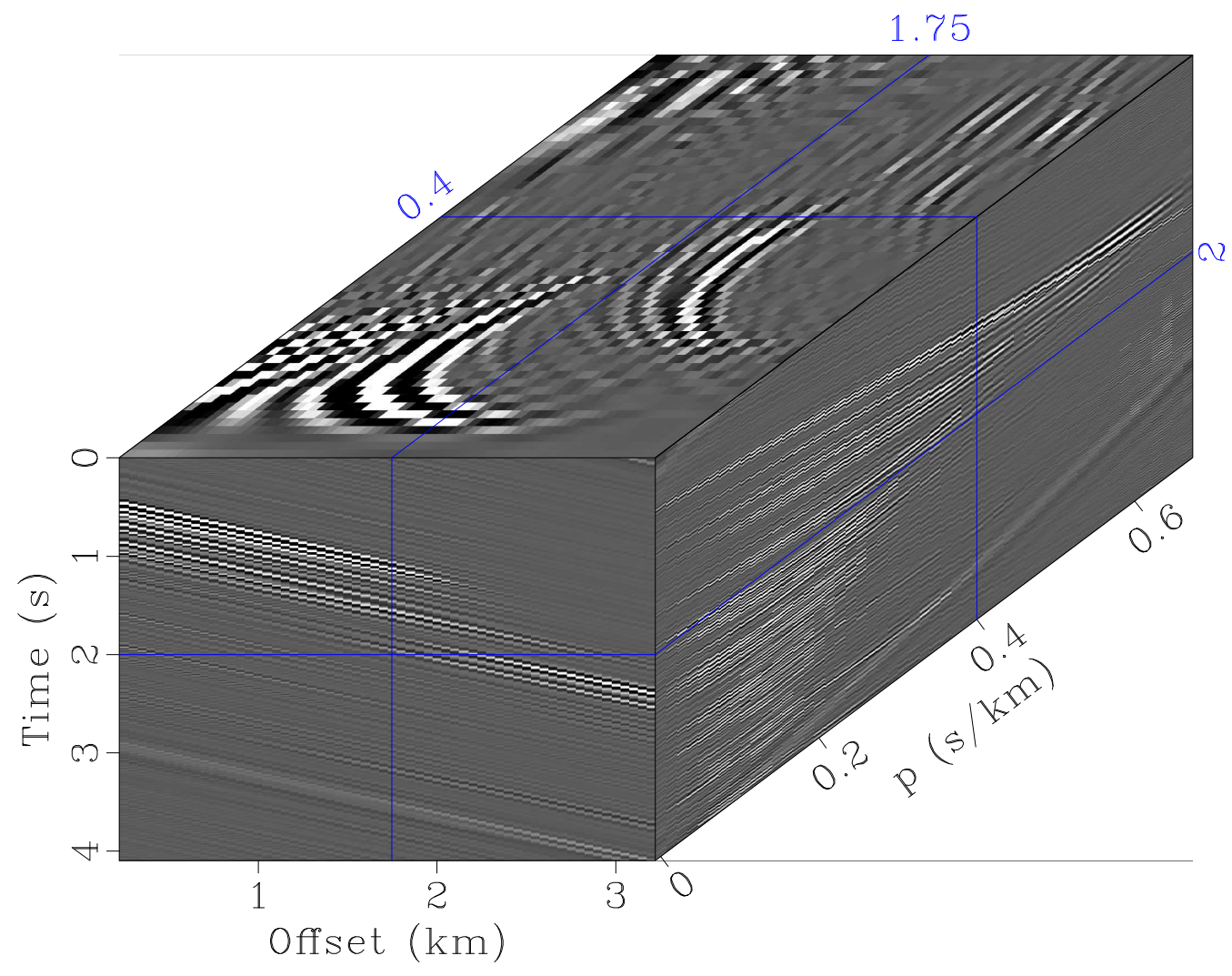

Figure 3.5: The CMP gather in the extended $t-x-p$ domain. Stacking over $p$ restores the gather in Figure 2.3. chapter3/haskell txp 


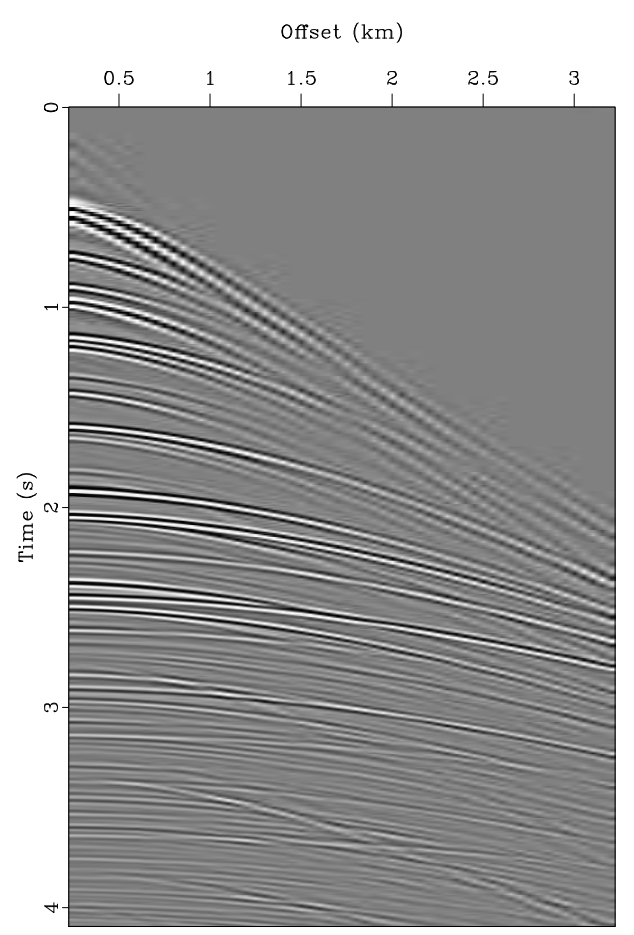

(a) Reconstructed gather

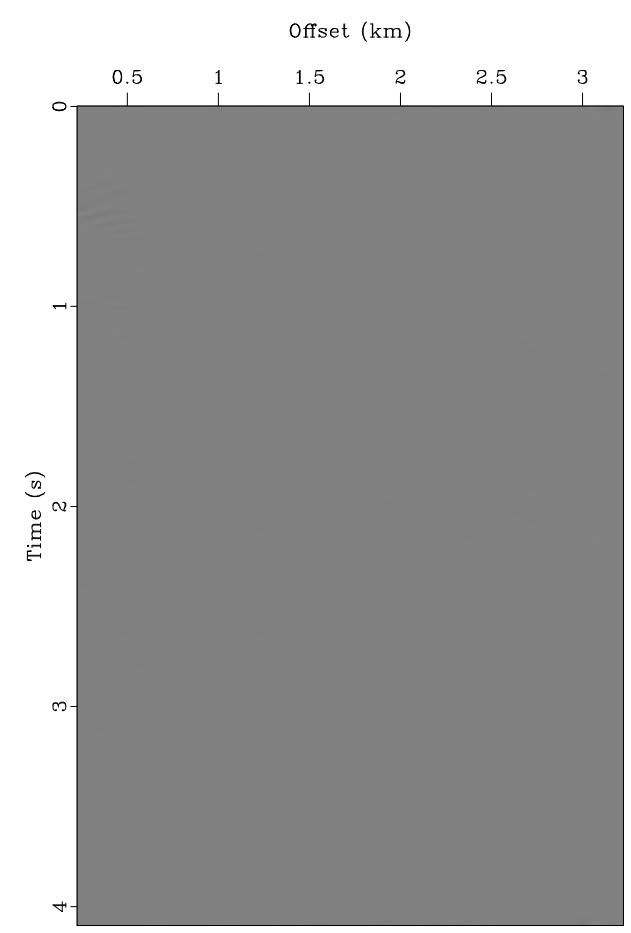

(b) Difference

Figure 3.6: (a) Reconstructed CMP gather, (b) Difference with original gather. chapter3/haskell recon 


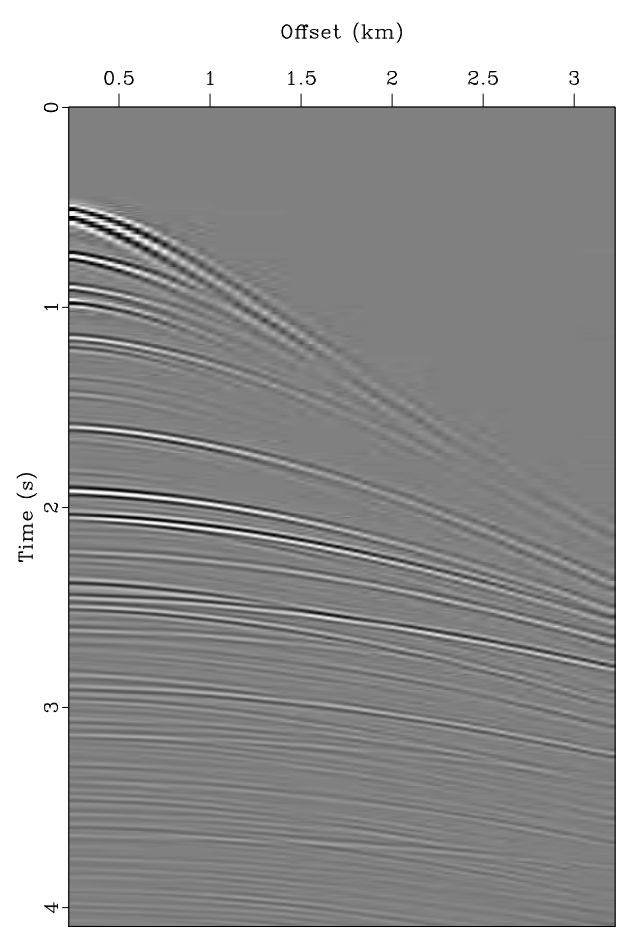

(a) Gather after demultiple

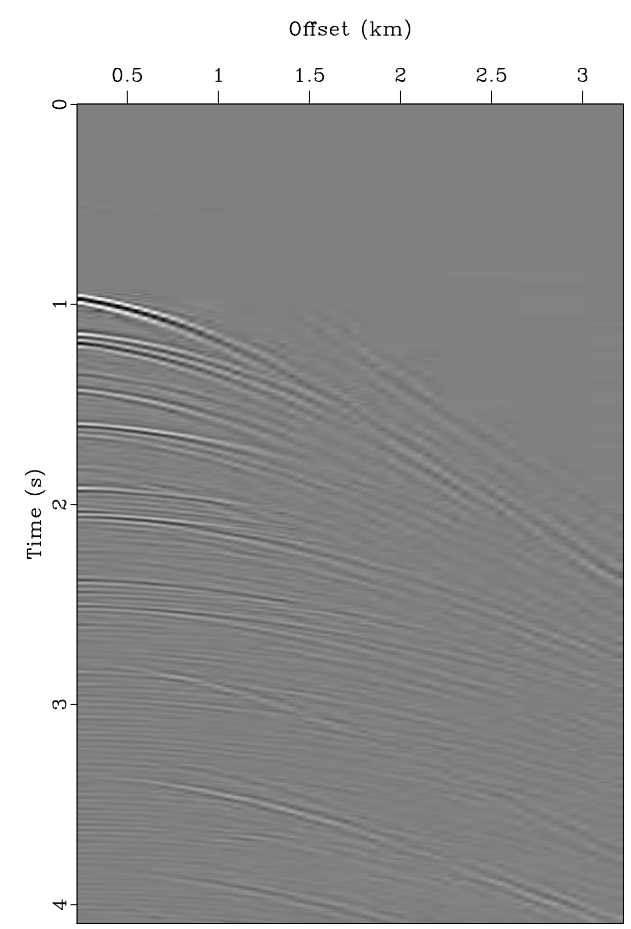

(b) Difference

Figure 3.7: (a) Gather after demultiple, chapter3/haskell2 demultiple

(b) Difference with original gather. 


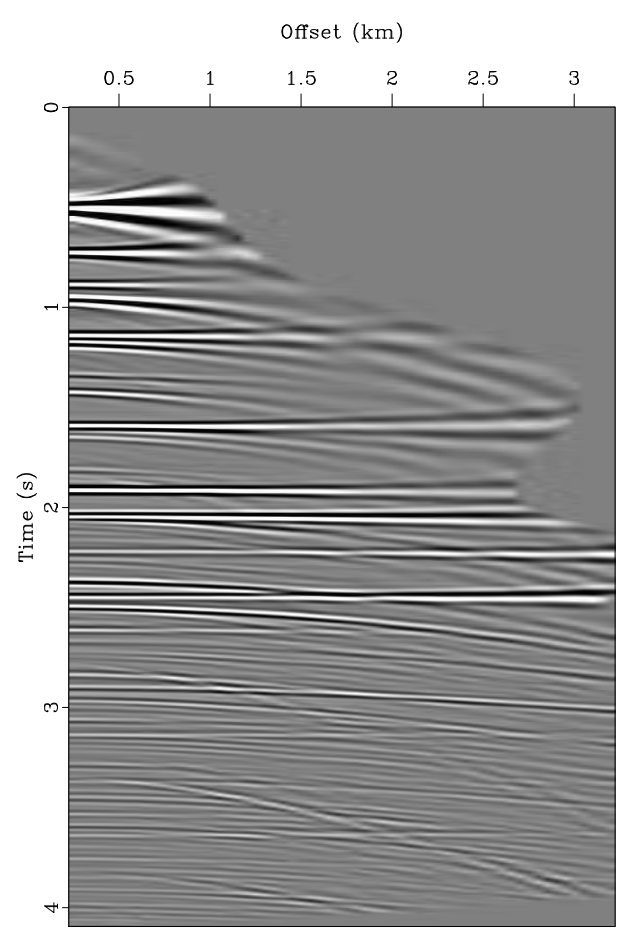

(a) NMO before demultiple

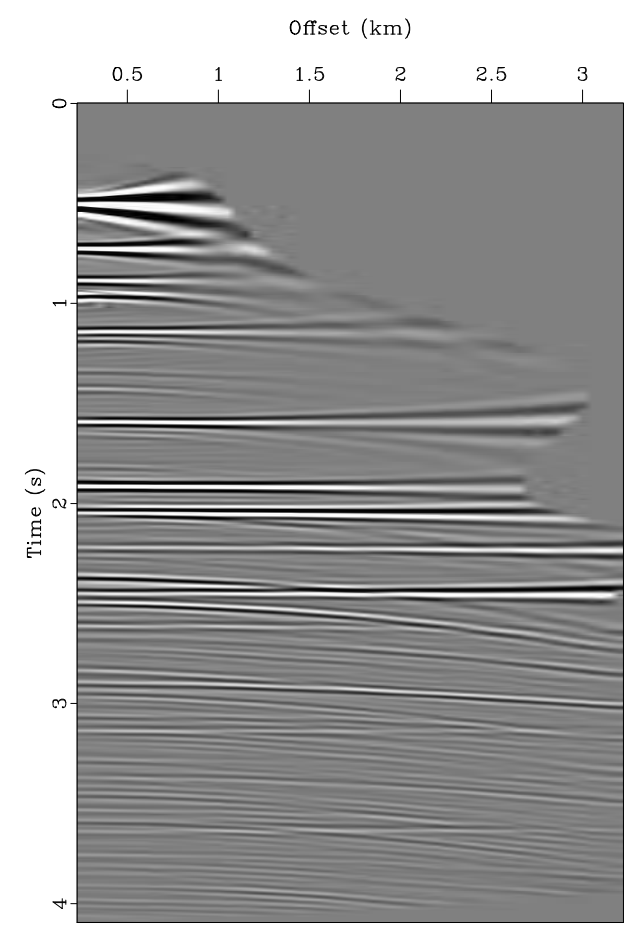

(b) NMO after demultiple

Figure 3.8: NMO corrected gather with picked primary velocity (a) before demultiple and (b) after demultiple. chapter3/haskell2 nmocompare 


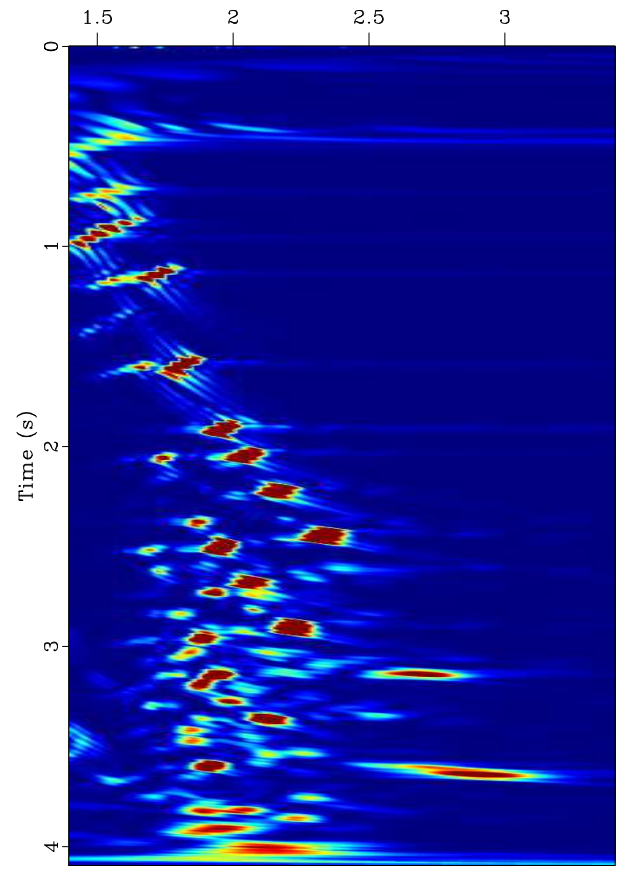

Velocity Scan

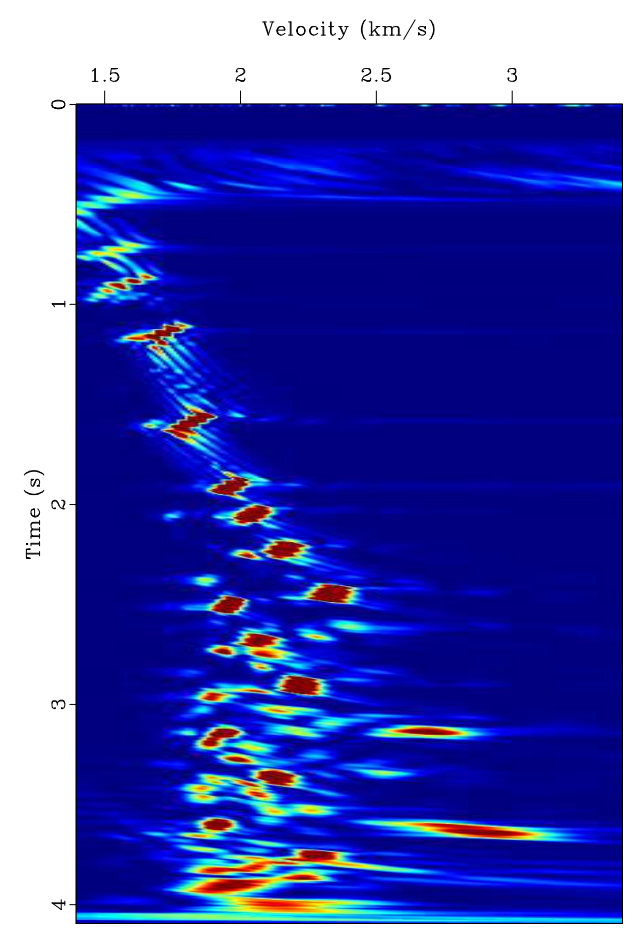

Velocity Scan

Figure 3.9: (a) Velocity scan before demultiple, (b) Velocity scan after demultiple. chapter3/haskell2 vscant 


\section{FIELD DATA EXAMPLE}

For a field data test, I take a Common mid-point (CMP) gather from the Gulf of Mexico. The gather has a $8 \mathrm{~s}$ recording and water bottom traveltime of $1.95 \mathrm{~s}$ (Figure $3.10 \mathrm{a}$ ). Since the water bottom traveltime is $1.95 \mathrm{~s}$, the first multiple should arrive at around 3.9s. The recording is shown from $1.5 \mathrm{~s}$ in Figures $3.10 \mathrm{a}$ and $3.10 \mathrm{~b}$. Both Figures $3.10 \mathrm{a}$ and $3.10 \mathrm{~b}$ have a gain applied to them. Figure $3.10 \mathrm{~b}$ shows the NMO corrected gather with the picked primary velocity. The first water bottom multiples at around $3.9 \mathrm{~s}$ are under-corrected as expected. Beneath the first order water bottom multiple, we observe other under-corrected events as well. Assuming that the picked primary velocity is reasonably correct, these under-corrected events represent first or higher order multiples. Figure 3.11 shows the CMP gather in the extended $t-x-p$ domain. Figures $3.12 \mathrm{a}, 3.12 \mathrm{~b}$ and 3.12 c show the original gather, the gather after multiple suppression and the filtered out portion respectively. The data has been windowed to display data after $1.5 \mathrm{~s}$ and a gain has been applied to make the deeper events more distinct. Figures $[3.13 \mathrm{a}$ and $[3.13 \mathrm{~b}$ show the velocity scans before and after multiple suppression. The velocity scans reveal that much of the multiples that are present in the original gather are suppressed. In addition, the primaries start to become more distinct after 3.9 s making it possible to pick a distinct trend while picking primary velocities. 


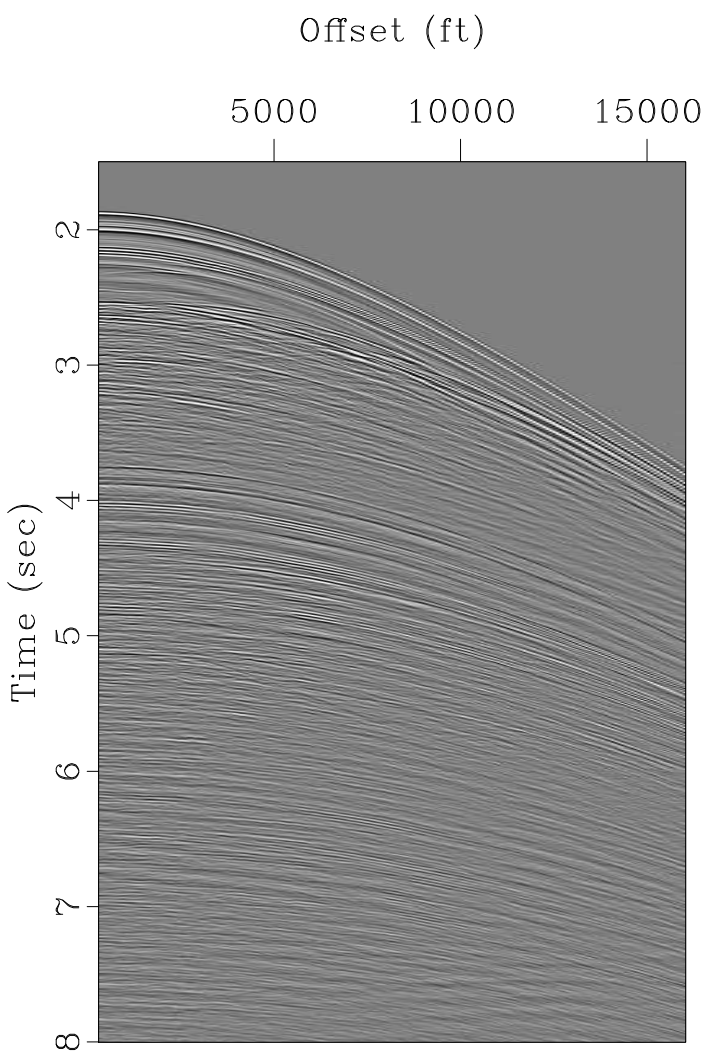

(a) Original gather

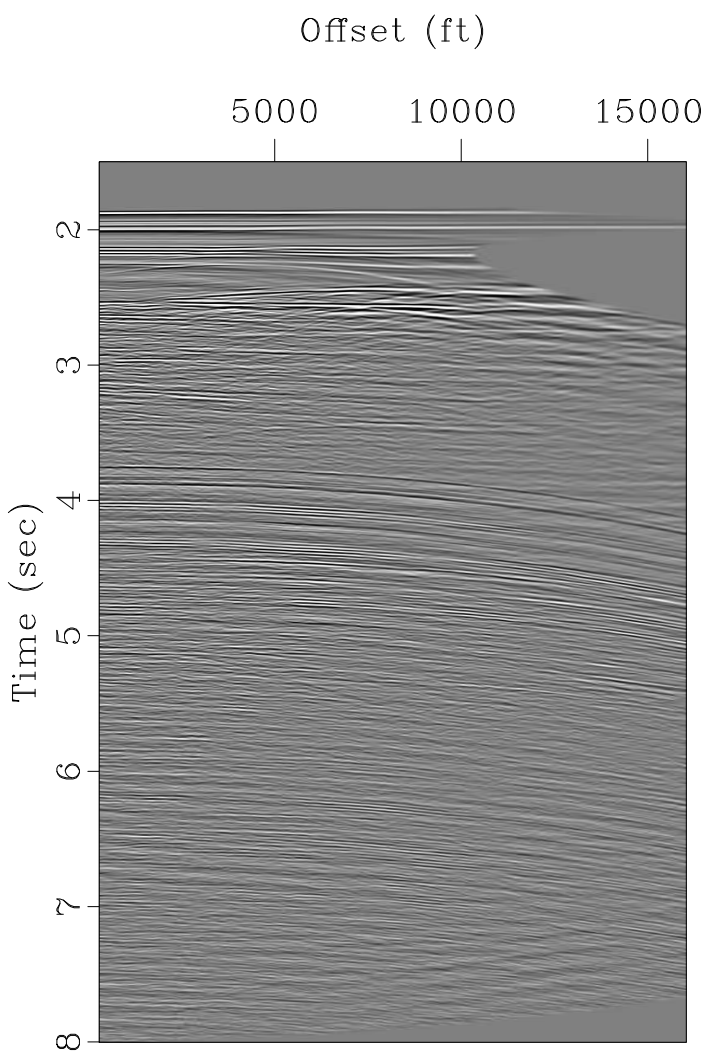

(b) NMO corrected gather

Figure 3.10: (a) Original CMP gather. (b) NMO corrected gather with picked primary velocities chapter3/gomgather ppt2 


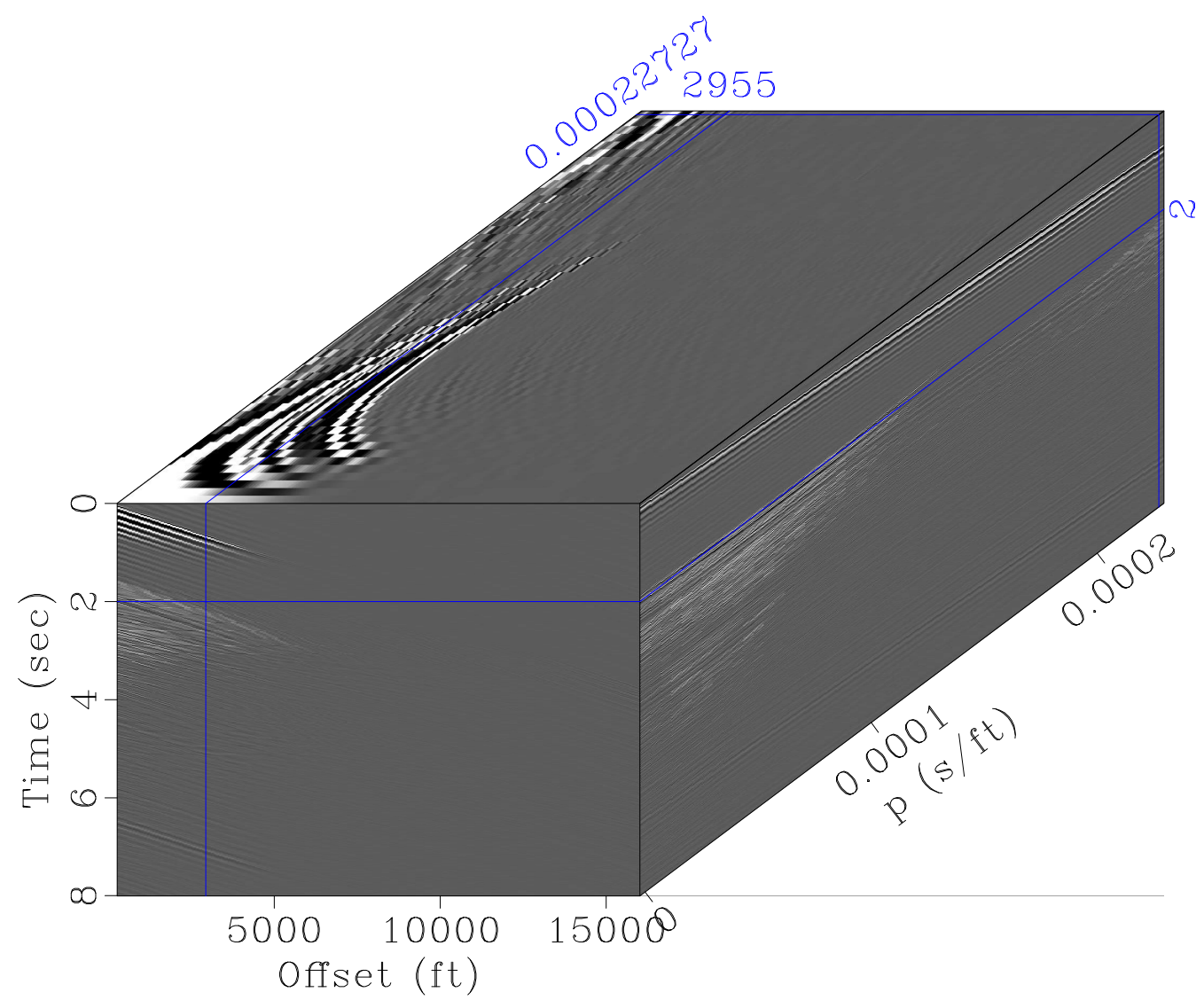

Figure 3.11: CMP gather in the extended $t-x-p$ domain chapter3/gomgather txp2 


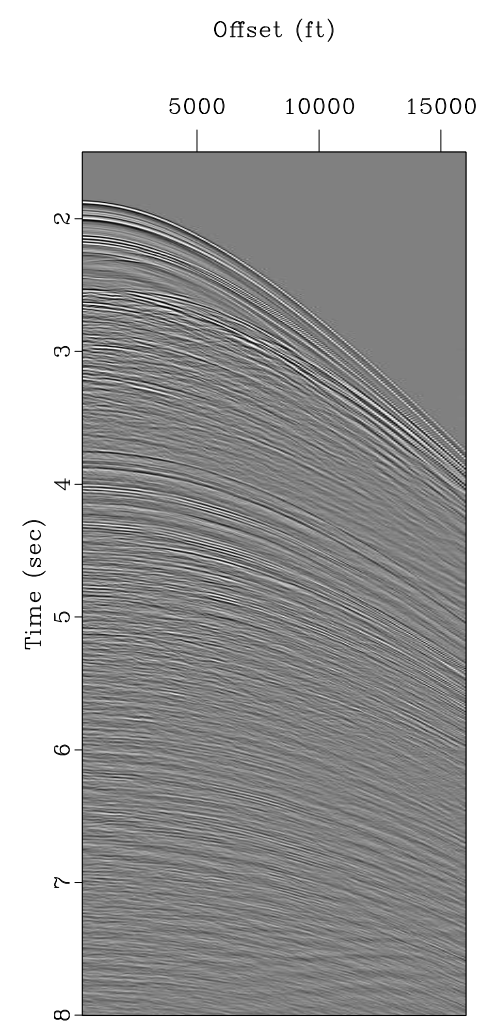

(a) Original gather

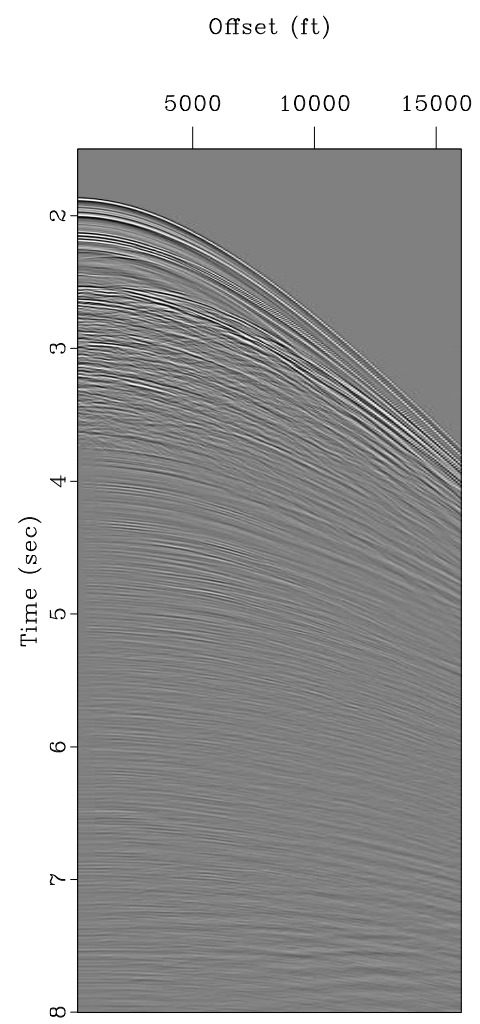

(b) Filtered gather

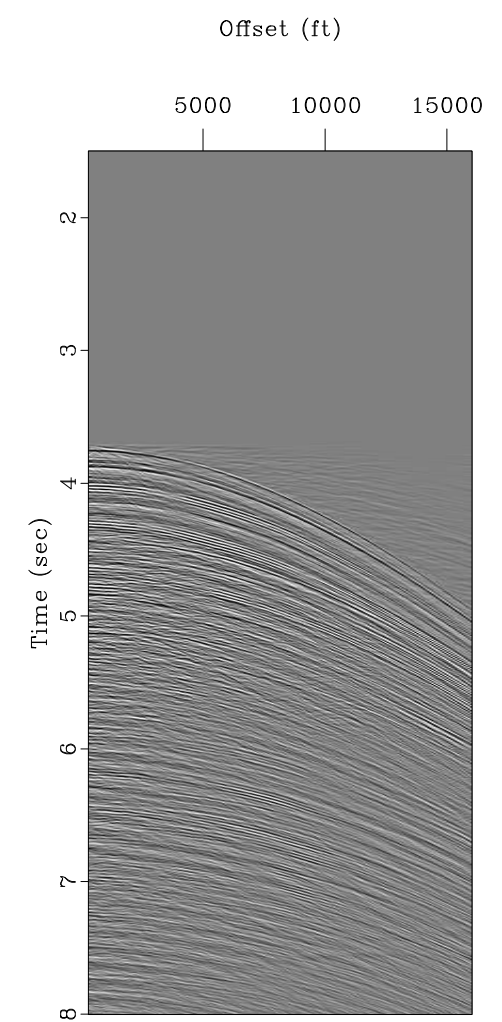

(c) Subtracted multiples

Figure 3.12: (a) Original gather. (b) Gather after multiple suppression. (c) Residual (filtered out portion). chapter3/gomgather resultposterbin 


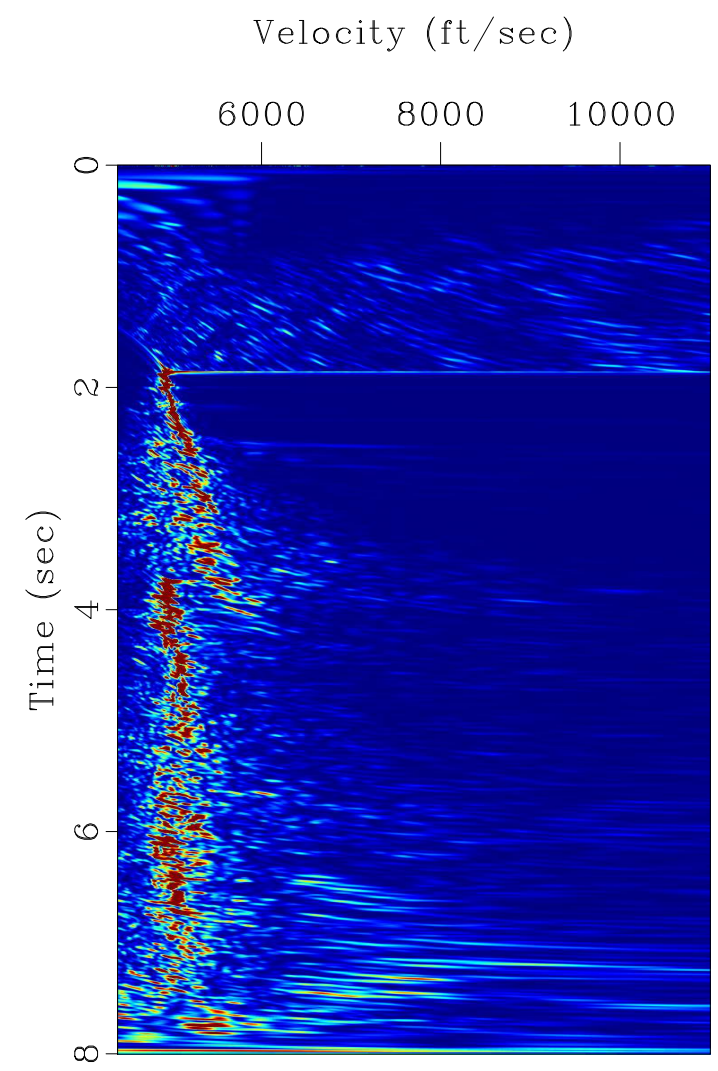

(a) Before suppression

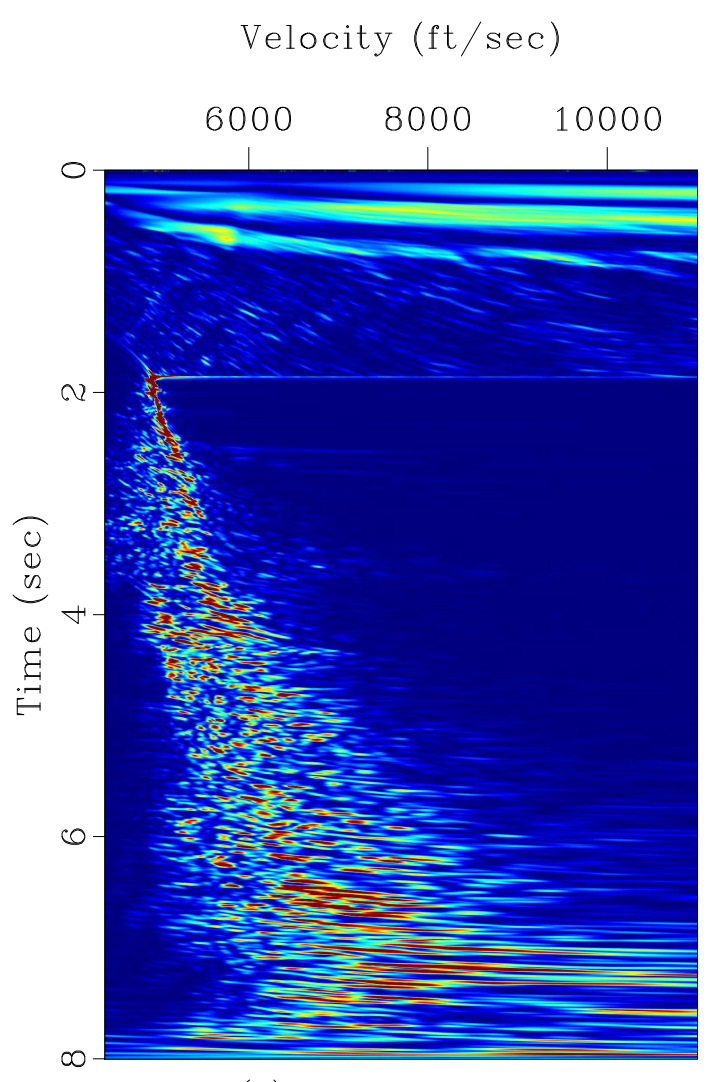

(b) After suppression

Figure 3.13: Velocity scan (a) before multiple attenuation. (b) after multiple attenuation. chapter3/gomgather vscanposter 


\section{NOISE SUPPRESSION EXAMPLE}

One of the advantages of the $t-x-p$ method is that it attempts to suppress anything that are not primary reflections. Therefore, it can be used to suppress not only multiples, but also any other event that does not follow the hyperbolic paths associated with the picked primary velocities. In this section, I demonstrate this by applying the method to ground roll suppression for the same gather demonstrated in the original paper. Figure 3.14 a shows a shot gather from Yilmaz's collection of

shot gathers (Yilmaz and Cumro, 1983) that contains a strong ground-roll noise. Figure 3.14b shows the gather after the filtering, and Figure 3.14 shows the portion that has been filtered out. It can be clearly seen in Figure $3.14 \mathrm{~b}$ that the algorithm has done an excellent job in suppressing multiples. Figure 3.14k, that displays the residual or difference between figures 3.14 and b, demonstrates that most of the primary signal has been preserved during the ground-roll suppression.

\section{DISCUSSION}

The examples shown in this chapter on pre-stack data show promising results for multiple suppression and versatility of the method to suppress other kinds of noise as well. In chapter 4, this multiple suppression technique is applied to the Viking-Graben data set and results for both pre-stack and stacked data are shown. In chapter 5 , I describe the advantages, disadvantages and the scope of this technique in seismic data processing on a production scale. 


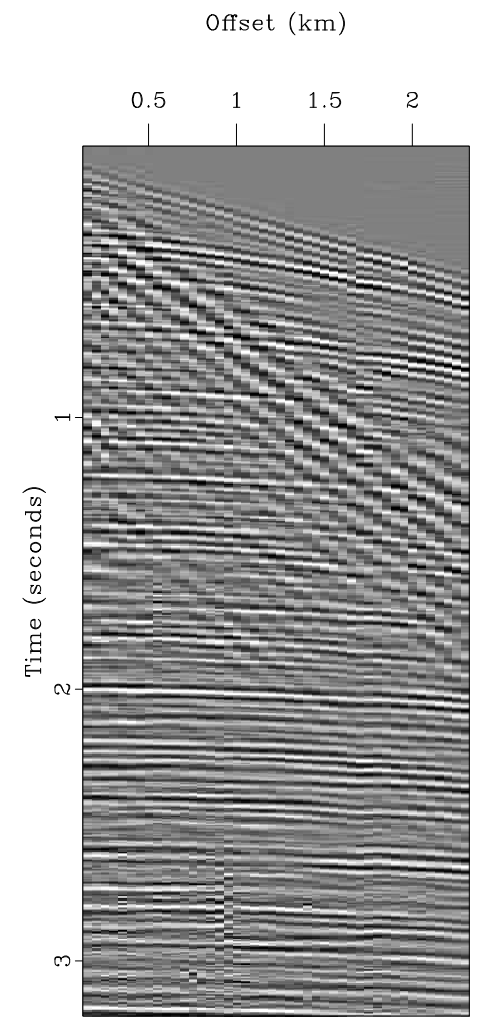

(a)

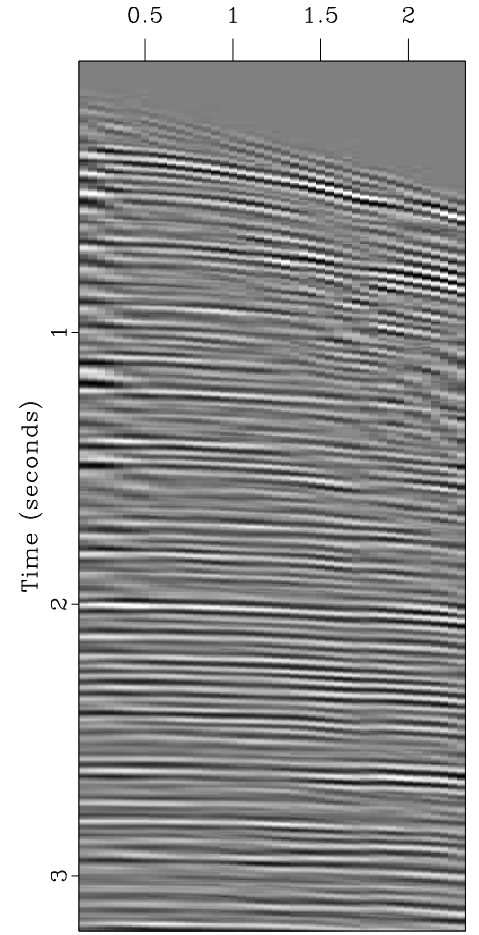

(b)

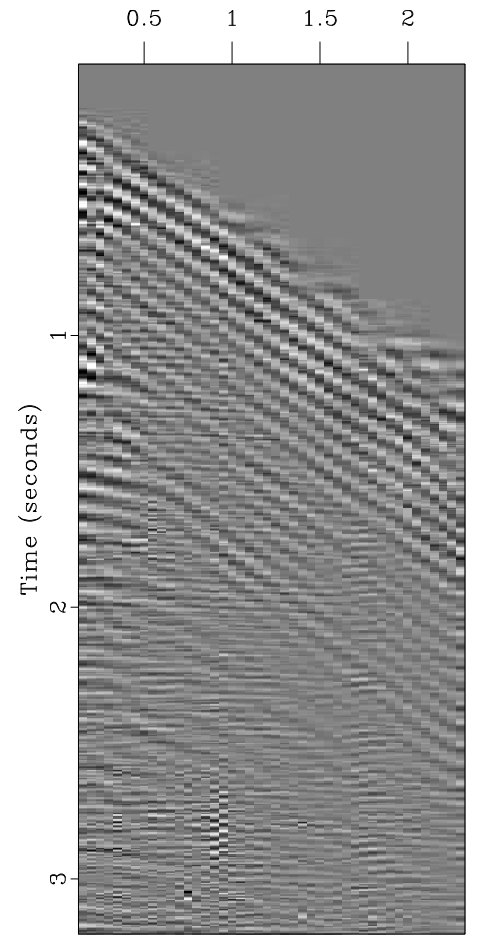

(c)

Figure 3.14: (a) Original CMP gather. (b) Gather after ground roll suppression. (c) Residual (filtered out portion). chapter3/data-gr finalresg 


\section{Chapter 4}

\section{Multiple attenuation of the Viking Graben data set}

\section{STUDY AREA}

In this chapter, I apply multiple suppression in the $t$ - $x$ - $p$ domain to line 12 from the Viking-Graben data set. This marine seismic data was acquired in the North Sea over a structure called the Viking Graben by Mobil Corporation and then released in 1994 to support development of seismic processing methods and for AVO studies (Keys and Foster, 1998). The line is a total of $25 \mathrm{~km}$ in length and the data includes pre-stack shot record data and measurements from two wells that intersect the line. The locations of these two wells are shown in Figure 4.3. This data set has been widely used to validate amplitude versus offset (AVO) studies as well as multiple suppression algorithms (Lumley et al., 1995; Keys and Foster, 1998, Madiba and McMechan, 2003).

The Viking Graben is a linear trough with a North-South trend, between the Norweigian and UK sectors of the northern North Sea. The map of the area is shown in Figure 4.1, modified from (Glennie, 1990). The Viking Graben formed during rifting during the Late Permian to Triassic. Episodes of extension and accompanying sedimentation continued through the Jurassic into the Early Cretaceous. Normal subsidence and filling became the primary depositional mechanism by the Late Cretaceous (Glennie, 1990; Keys and Foster, 1998).

The stratigraphy of the reservoir rocks is shown in Figure 4.2. Reservoirs occur in the Frigg, 
Cod, Stratfjord and Brent formations. A significant unconformity occurs at the base of the Cretaceous. This is labelled as BCU and is shown in Figure 4.3. The Jurassic was a period of active faulting. The hydrocarbon traps are usually fault bounded structures, but some are associated with the stratigraphic truncation at the BCU (Glennie, 1990; Keys and Foster, 1998).

The depositional environment of the Jurassic reservoirs range from fluvial to deltaic to shallow marine (Keys and Foster, 1998). Productive sounds have been found in diverse local structural positions, such as crests of tilted sands, downthrown sides of faults and arched-over salt-induced "highs" (Glennie, 1990).

\section{DESCRIPTION OF DATA SET}

As specified earlier, the data set contains prestack shot records and measurements from two well locations shown in Figure 4.3 . The shot records have a recording length of $6 \mathrm{~s}$ with $4 \mathrm{~ms}$ sampling interval, i.e, there are 1501 samples in the time direction. The offset/receiver group spacing for the shot records is $25 \mathrm{~m}$ each with a total of 120 offsets recorded, indicating a recording cable length of $3 \mathrm{~km}$. The near-offset (distance between source and first receiver) is $262 \mathrm{~m}$. Shot numbers 300,400 , 500, 600 and 700 from the line are shown in Figure 4.4.

The water bottom is fairly flat, with the water bottom traveltime being around $0.5 \mathrm{~s}$ for the entire line. The near-offset section shown in Figure 4.5, demonstrates this fact. A close examination of Figure 4.5 demonstrates that there are a lot of multiples in the data. Many of the events seem 


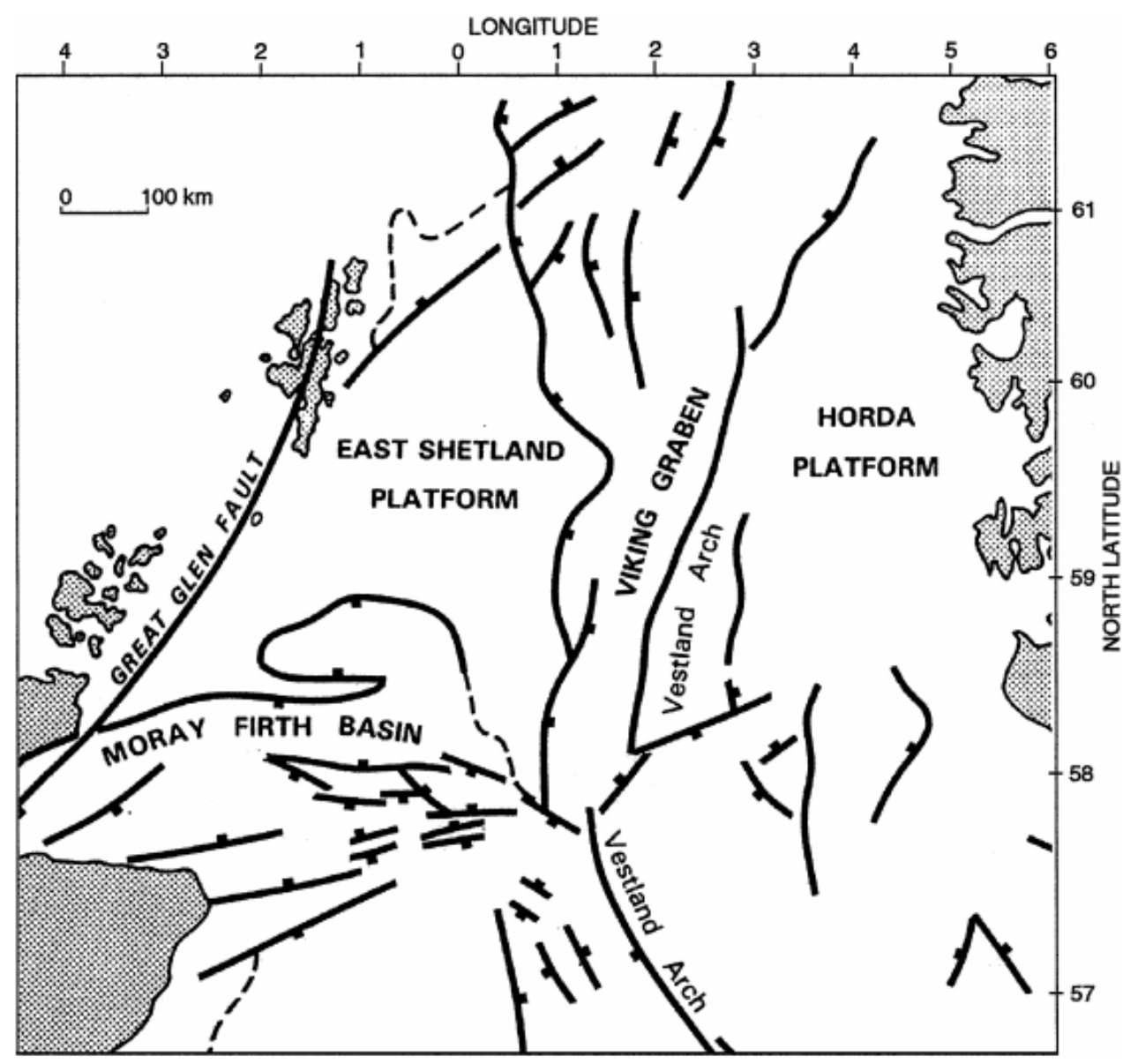

Figure 4.1: Map of the Viking Graben Area, modified from Glennie (1990). chapter4rough/viking viking-graben 


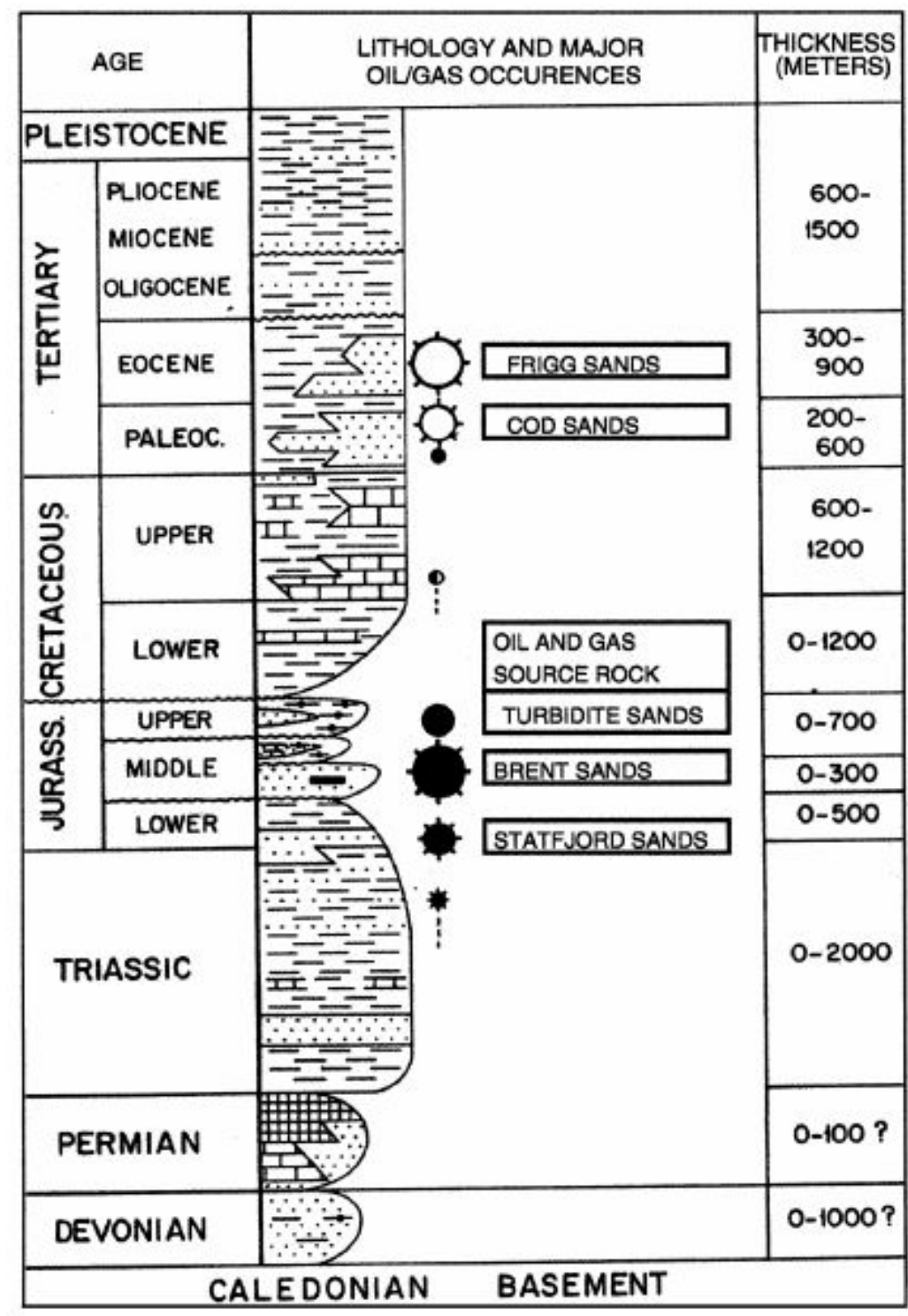

Figure 4.2: Stratigraphic column of the northern North Sea reservoir rocks (Glennie (1990)). chapter4rough/viking strat 


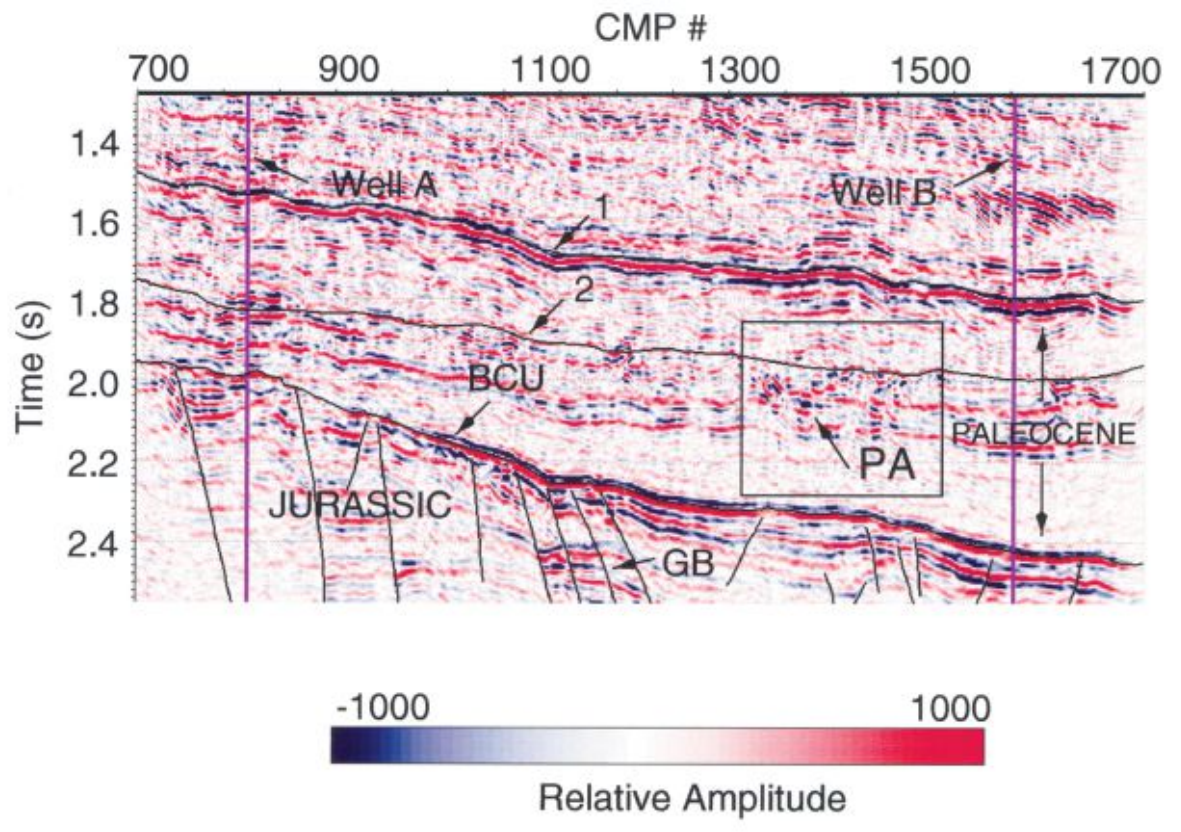

Figure 4.3: Migrated section. The major unconformity at the sase of the Cretaceous is located at $1.97 \mathrm{~s}$ at Well A and 2.46s in Well B (Madiba and McMechan (2003)). chapter4rough/viking bcu 
to be repeated multiple times right up to the entire recording length.

After conversion from shot gathers to Common-Midpoint (CMP) gathers, the data set has a trace spacing of 50m, 60 offsets and a total of 2143 CMP gathers. Figure 4.6 shows CMPs 201, 401, 601, 801, 1001 and 1201 from the line and Figure 4.8 represents the same gathers with Normal Moveout (NMO) correction applied with the picked primary velocities .

A close look at the velocity scans in Figure 4.7 shows that there are a lot of multiples. The velocity scans corresponding to CMPs 201, 401, 601 and 801 reveal very strong first order water bottom multiples: to the extent that any other event with a higher moveout velocity is almost indistinguishable. The velocity scans corresponding to CMPs 1001 and 1201 show the presence of first order water bottom multiples, as well as, other multiples and possibly some primaries.

The NMO corrected gathers in Figure 4.8 show more or less flat events for data corresponding to the first 1s. One interesting point to note is that for CMPs 201, 401 and 601, there is an event at around $1.97 \mathrm{~s}$ that appears completely flat (interfering with under-corrected first order water bottom multiples). This bears testimony to the fact that my velocity picks are reasonably accurate. One must note that for multiple suppression in $t-x-p$ domain to work, the picked primary velocities only need to be reasonably accurate. The $t-x-p$ tapering filter coeffcients are robust enough to deal with slight inaccuracies in picked primary velocities.

Figure 4.9 reveals the picked primary and interpolated velocities for the entire line. I picked the velocites for every 100th CMP gather and then interpolated them for the entire line. 

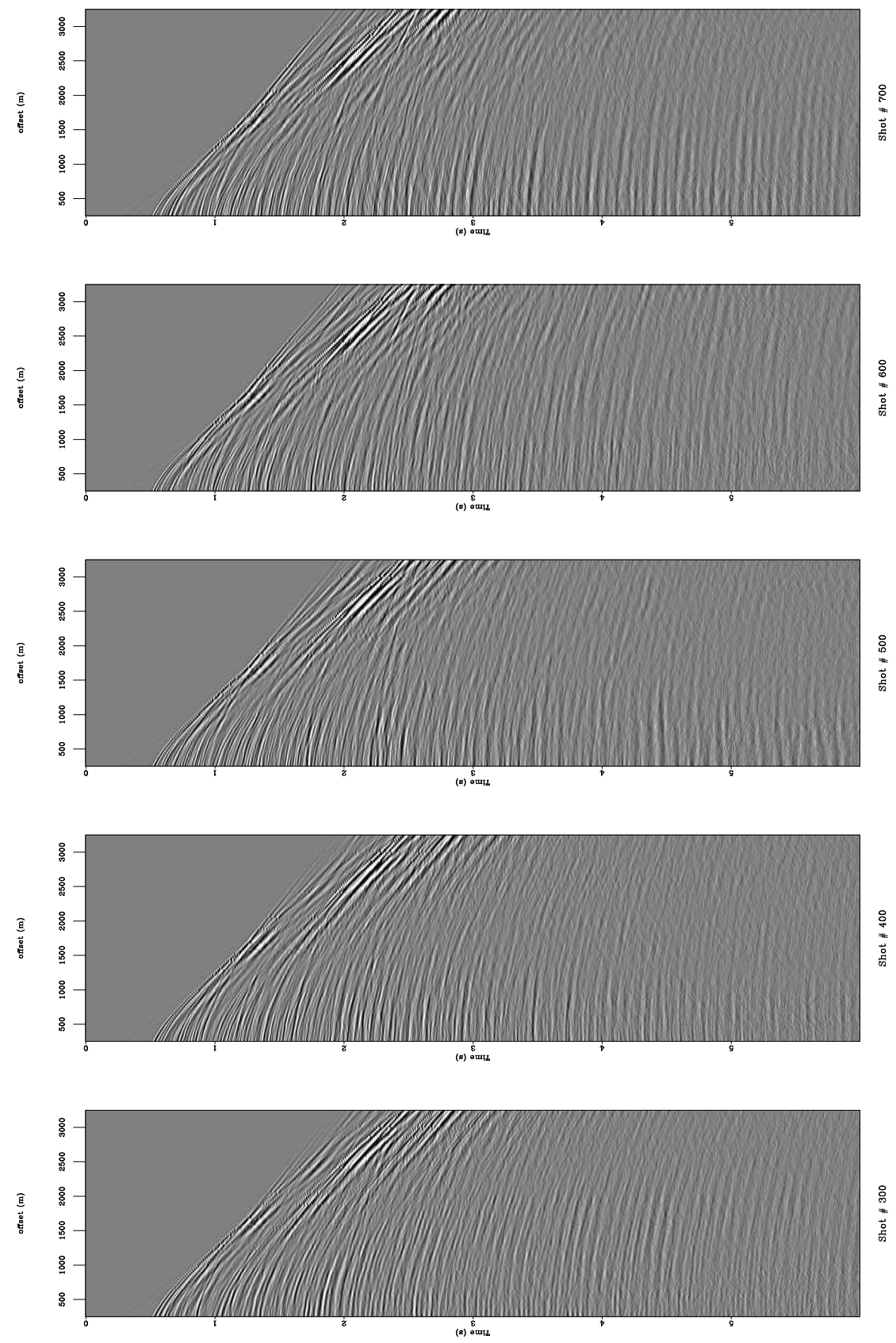

Figure 4.4: Shots 300, 400, 500, 600 and 700 from the data line. chapter4rough/viking someshotgathers 


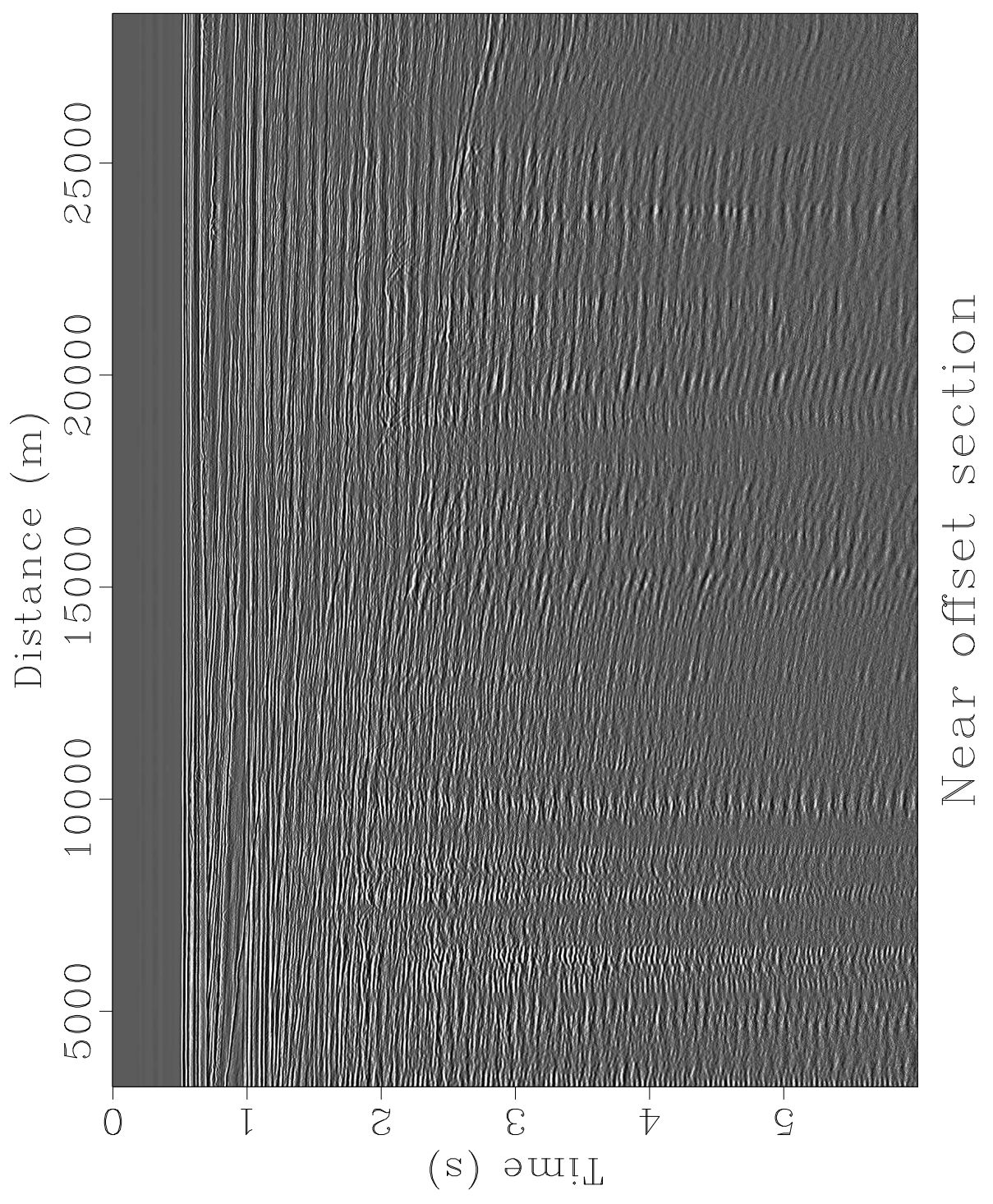

Figure 4.5: Near offset section for the entire 25km Viking Graben line. chapter4rough/viking near-offset 

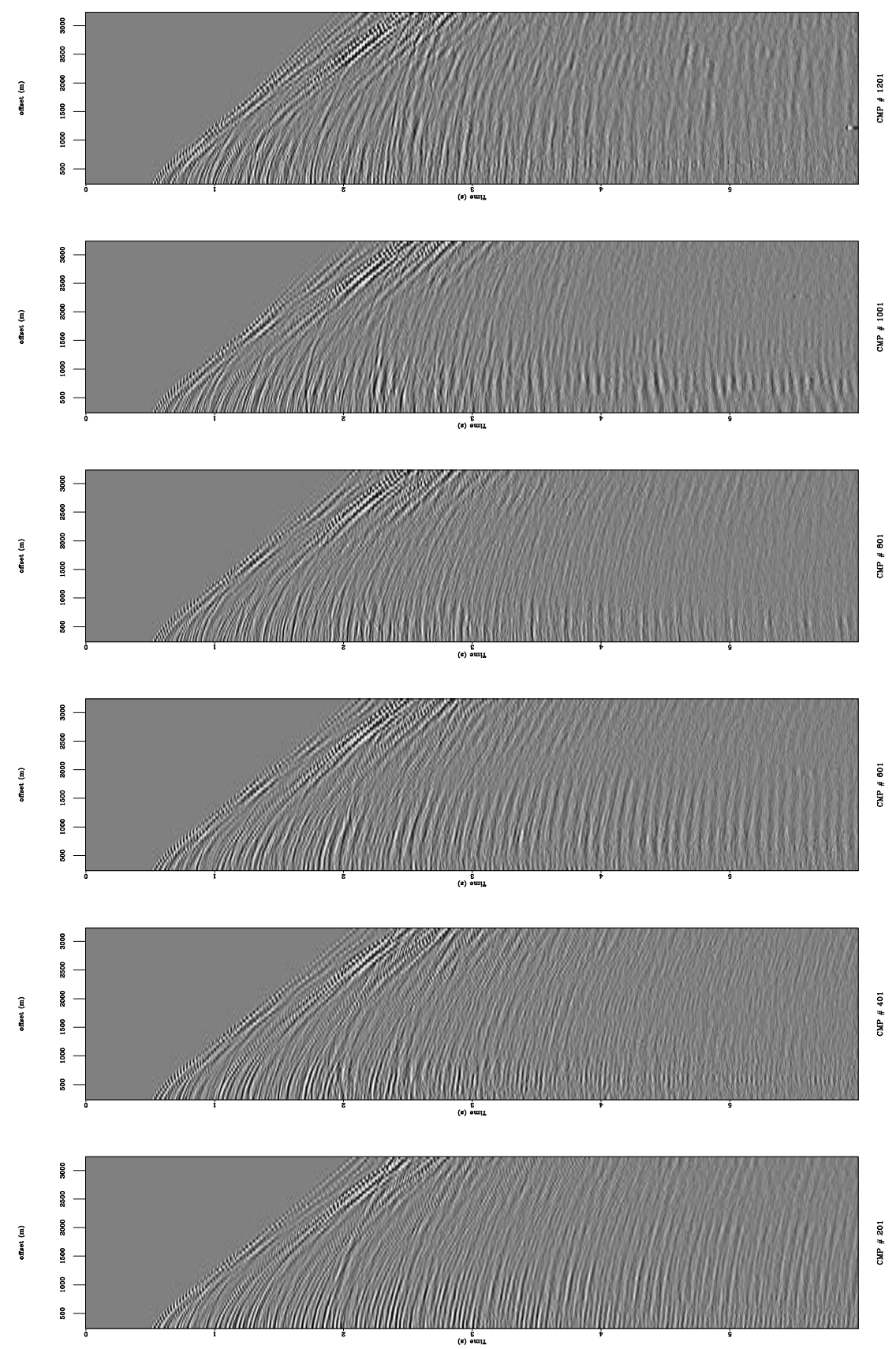

Figure 4.6: CMPs 201, 401, 601, 801, 1001 and 1201 from the data line. chapter4rough/viking somecmpgathers 

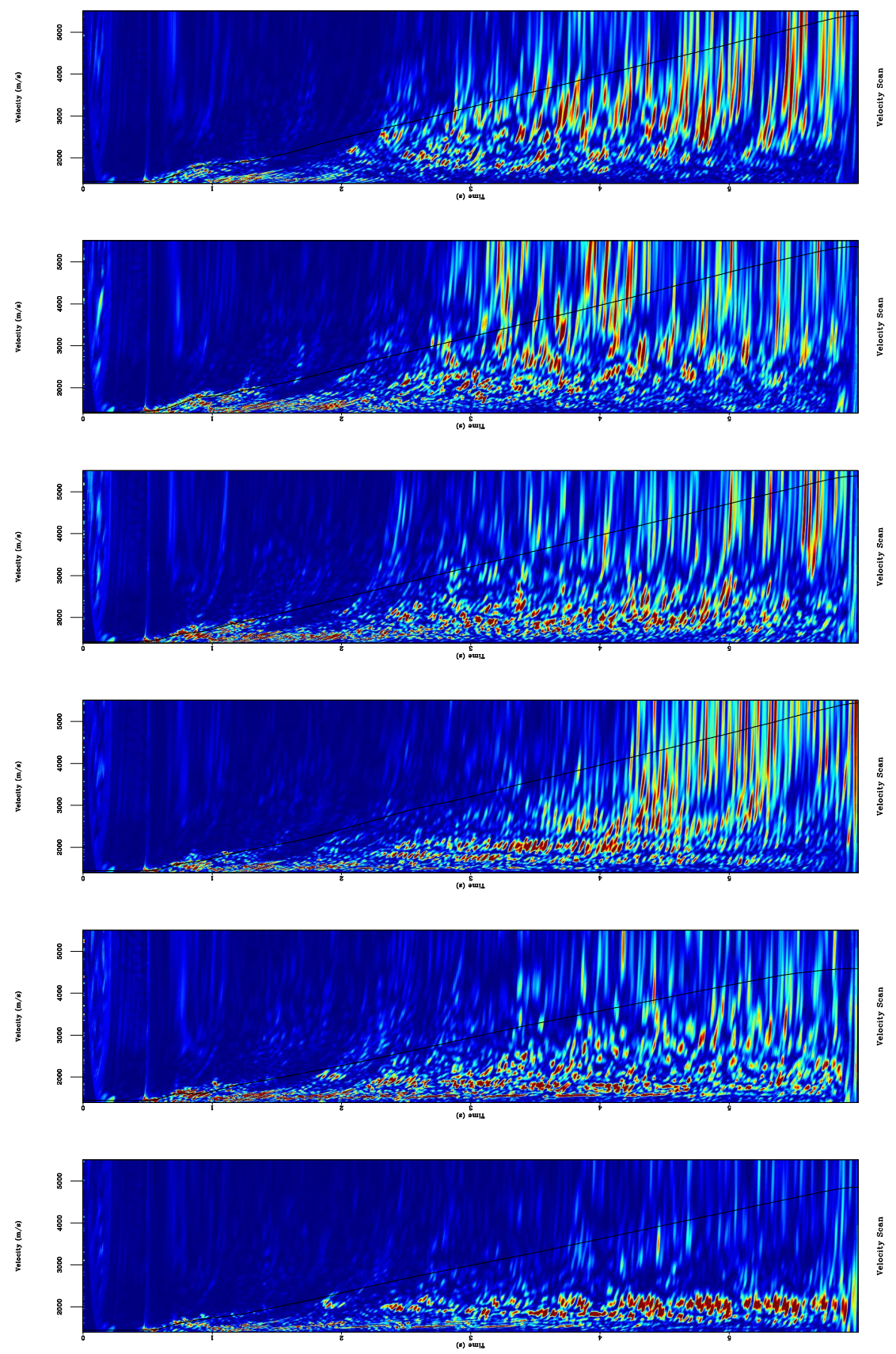

Figure 4.7: Velocity scans with picked primary velocities for CMPs 201, 401, 601, 801, 1001 and 1201 from the data line. Though in some of the gathers, the primaries for the deeper parts are incomprehensible, velocity after demultiple reveals reasonable accuracy of the picked velocities here. chapter4rough/viking vscansome 

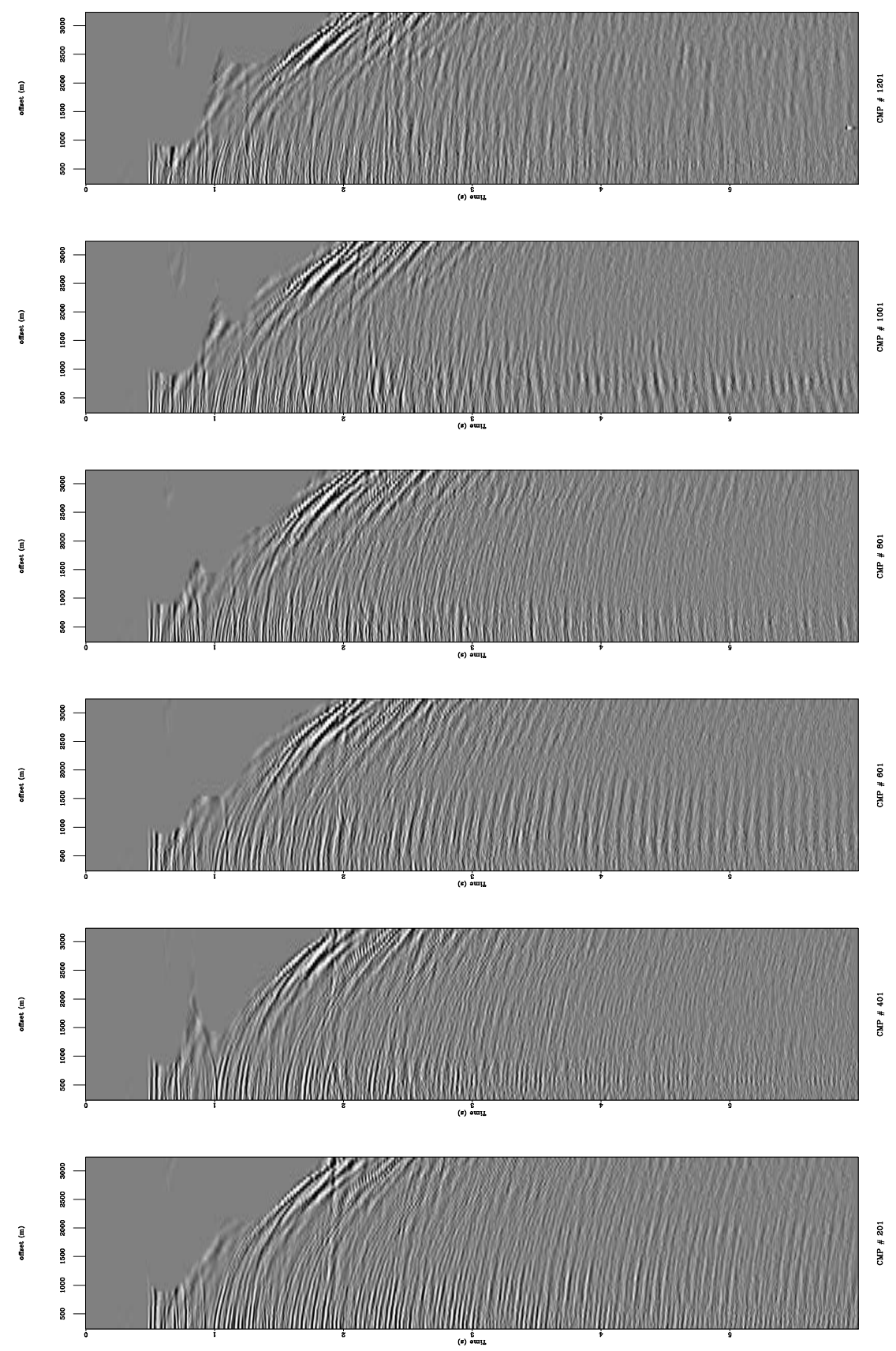

Figure 4.8: NMO corrected gathers for CMPs 201, 401, 601, 801, 1001 and 1201 from the data line. chapter4rough/viking somenmo 


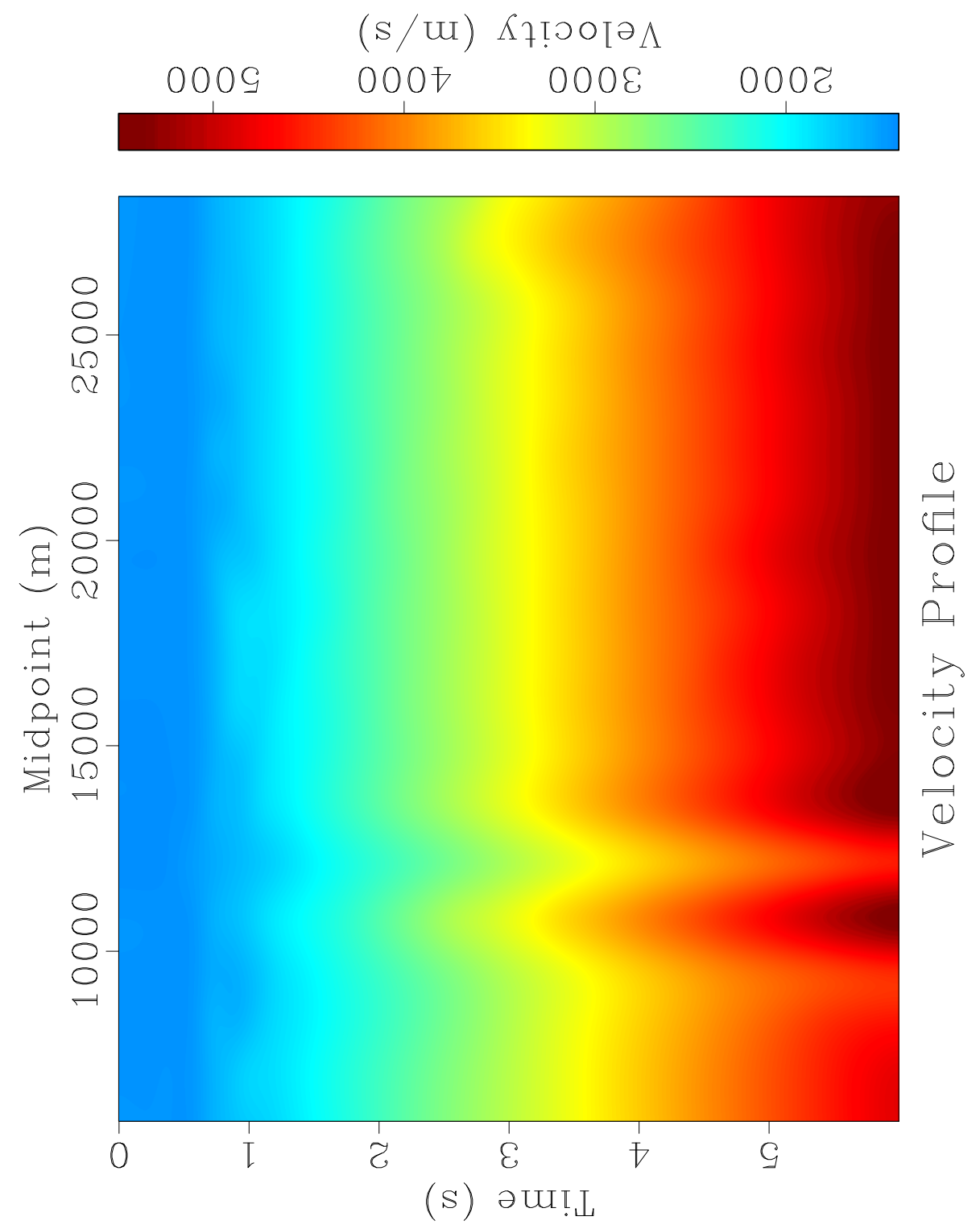

Figure 4.9: Picked primary velocity for the entire line. chapter4rough/viking vel0 
Figure 4.9 shows that the picked primary velocity model is smooth. The velocity profile does not show lateral variation in velocities (which might be in slight contradiction to earlier studies), but this velocity model is acceptable as our velocities only need to be reasonably accurate.

After conversion from shot to CMP gathers, the first 120 CMPs and the last 120 CMPs do not have complete foldage. For all demultiple operations I have used CMPs 201 through 2001. All of these CMPs have complete fold. From here on, whenever I mention the words "entire line", I will be referring to most CMPs having complete fold, i.e, CMPs 201 through 2001. The stacked sections however, show appropriate co-ordinates for distance along the second axis because of information being stored in the headers.

Figure 4.10 shows the Normal Moveout (NMO) stack for the first 3s of data. Upon close observation, we do not see much multiples that were apparent in the near-offset section (Figure 4.5). This is because the stacking operation itself behaves like a demultiple filter and kills much of the multiples (specially at far offsets where the difference in moveout or velocity between primary and multiple reflections are significant). The brute stack with water velocity (Figure 4.11 is however an interesting section to look at to prove the presence of strong multiples in the data. A close inspection of Figure 4.11 or a comparison between Figure 4.11 and Figure 4.10 shows significant presence of multiples. Most of the events that can be seen in the stack with picked primary velocities (Figure 4.10) can not be seen in the brute stack (Figure 4.11) because of the presence of multiples (couple with an inappropriate stacking velocities). Likewise, the brute stack (Figure 4.11) clearly shows repetition of certain events which are water bottom multiples, though the stack with primary velocities do not show any identifiable multiples. Later in the chapter, I will present the difference 
stack sections before and after demultiple which shows significant amounts of multiples removed.

\section{DEMULTIPLE RESULTS FOR THE VIKING GRABEN DATA SET Demultiple in $t-x-p$ domain}

I applied multiple suppression in the $t-x-p$ domain to the Viking Graben data set. Since separation of primaries and multiples are difficult at near offset, I applied a combination of threshold filtering (goes to zero for a value below a lower threshold and unity for a value above a certain threshold) and appropriate smoothing in the second and third dimensions inside the $t_{0}-x-p$ cube to eliminate as much multiples as possible while preserving the maximum amount of primaries.

Figure 4.12 shows gather numbers 201, 401, 601, 801, 1001 and 1201 after demultiple in the $t$ $x$ - $p$ domain. It is clear that much of the multiples have been removed from these gathers. This is further reflected in Figure 4.13 that shows the velocity scans for the same gathers after demultiple. I used mild filter co-efficients to achieve a decent balance between killing primaries and multiples. It is clear that some of the events that were difficult to distinguish in the velocity scans before demultiple in Figure 4.7 are quite clear in the velocity scans after demultiple (Figure 4.13). This is especially true towards the beginning of the line (towards CMP number 201). One interesting observation is that there is an internal multiple that keeps appearing at the left part of the section in the velocity scan. This is probably indicative of a thin layer of very high impedance that gradually thickens towards the later (right) part of the line. It is plausible that such a layer exists and does not allow either the upgoing or downgoing wavefield to escape, creating a series of intrabed multiples that appear in the velocity scans and are also apparent in the near offset-section (Figure 4.5). 


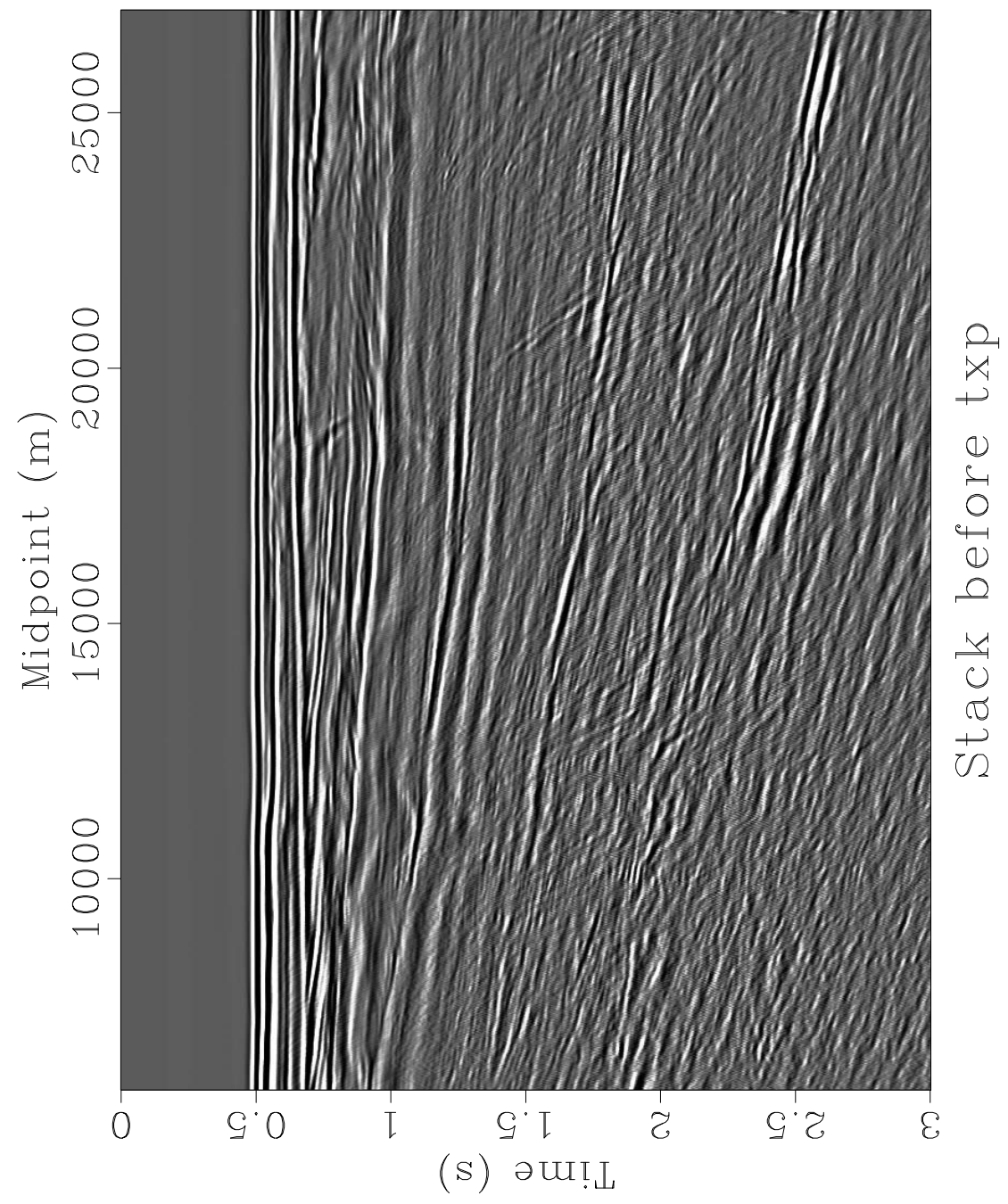

Figure 4.10: Normal Moveout (NMO) stack with picked primary velocity for the entire line for the first 3s. chapter4rough/txpbeforesrme stack201-2001 


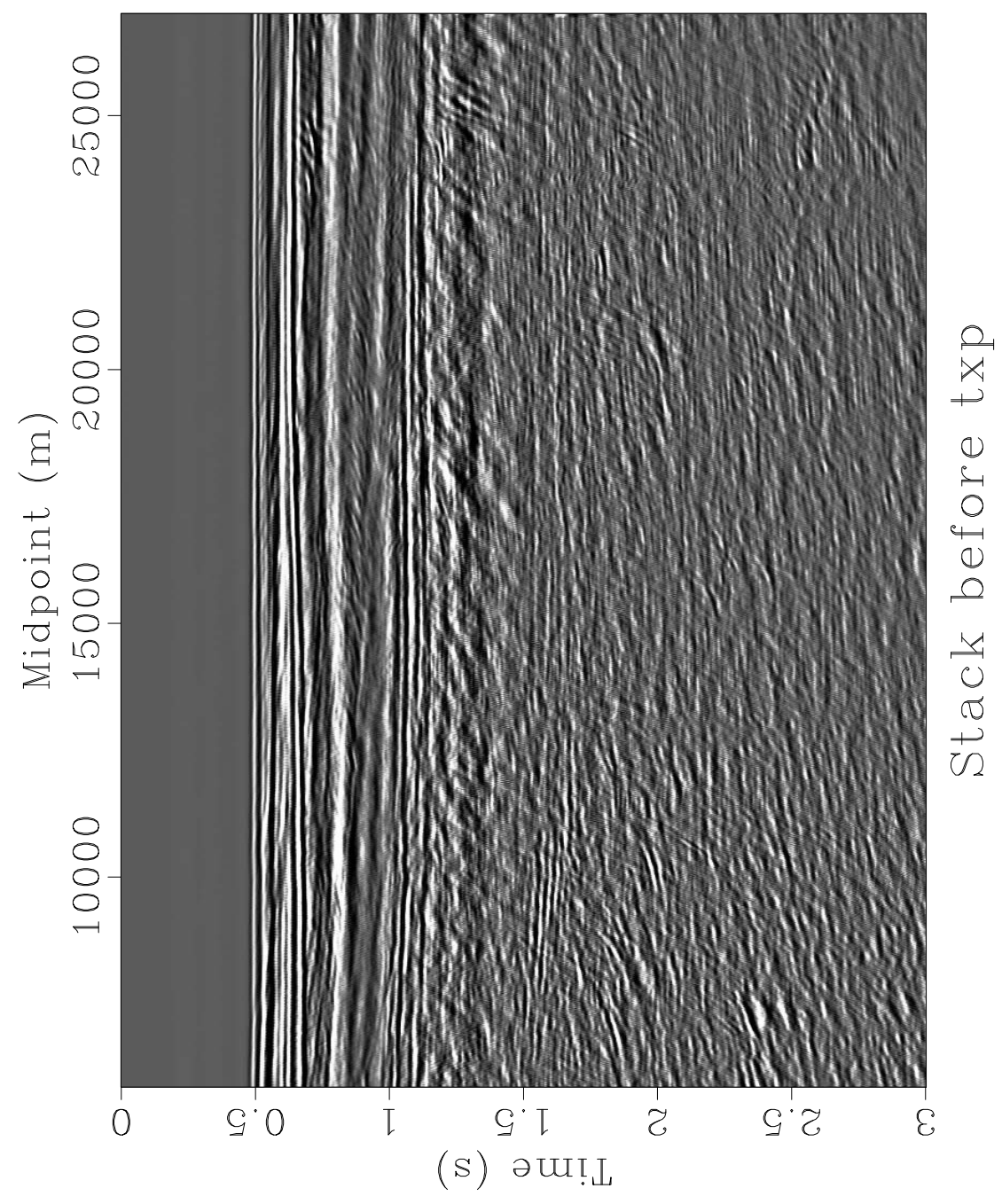

Figure 4.11: Normal Moveout (NMO) stack with water velocity for the entire line for the first 3s. chapter4rough/txpbeforesrme stack201-2001-1500 
An important point to note is that since separation of primaries and multiples are difficult at near offset, it is advisable to first use some prediction based method like WEREM (Lokshtanov, 2000) and/or surface related multiple elimination (Verschuur et al., 1988) that work well for near offset before filtering multiples in the $t-x-p$ domain.

Figure 4.14 shows the stacked section for the first 3s after demultiple and Figure 4.16 shows the stack with the water velocity after demultiple. While there are not much distinguishable differences between the stacks before (Figure 4.11) and after (Figure 4.14) because the stacking operation kills much of the multiples anyways, a look at the difference stack (Figure 4.15 and the brute stacks (with water velocity) before (Figure 4.11) and after (Figure 4.16) demultiple clearly show that after demultiple most of the flattish water bottom multiples have been suppressed to an appreciable extent. The difference stack (Figure 4.15) clearly shows that a significant amount of multiples have been suppressed and there is almost no leakage of primaries in the demultiple operation. This implies good separation between primaries and multiples in this domain. Also, a comparison between the brute stacks before (Figure 4.11) and after (Figure 4.16) demultiple shows that some of the dipping primary reflected events that were indistinguishable before demultiple become apparent after the demultiple procedure. I have purposely been mild with the filter coefficients so as to leave the first order water bottom multiple to be killed by SRME, which I show in the next section. 

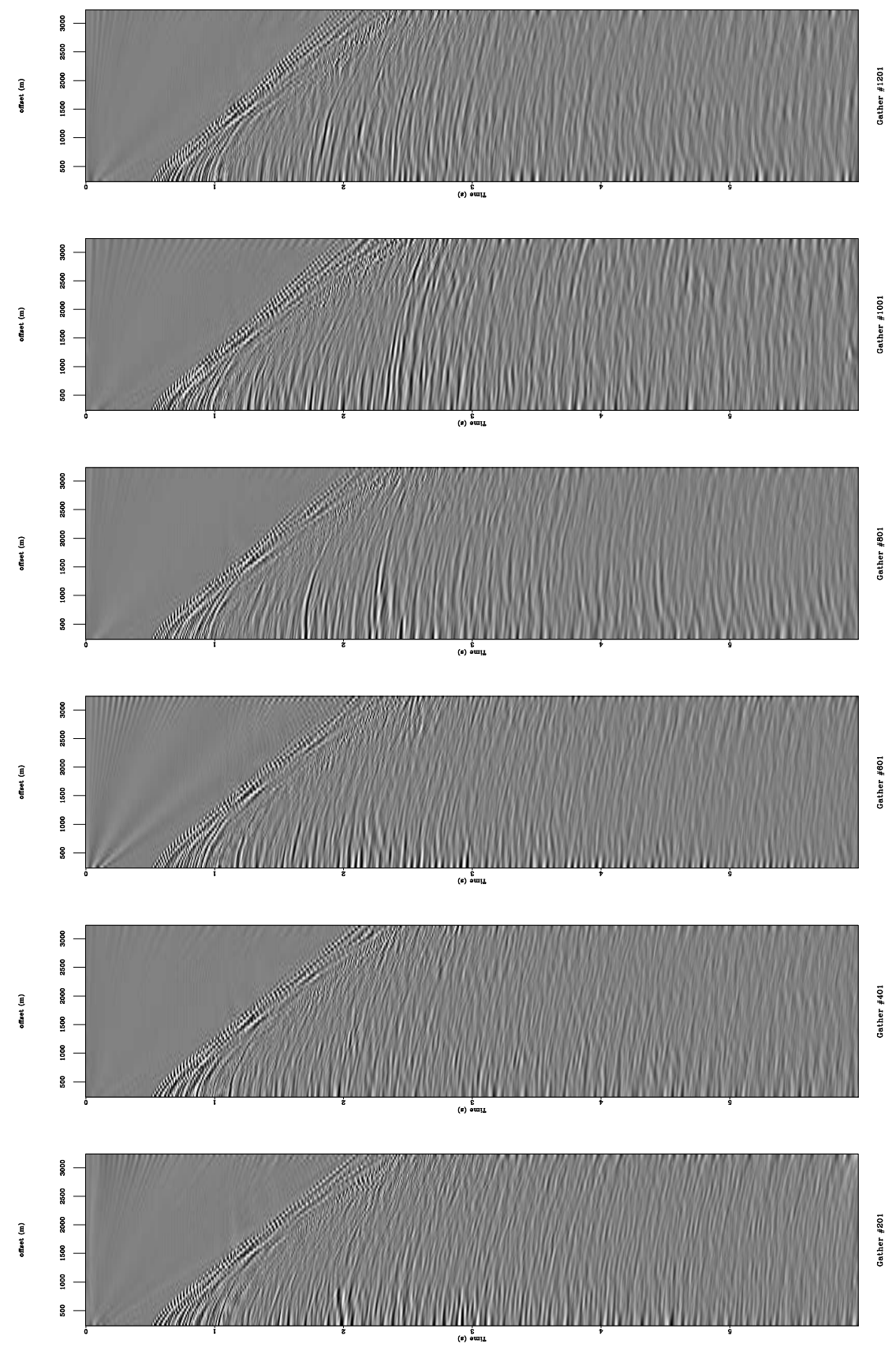

Figure 4.12: CMPs 201, 401, 601, 801, 1001 and 1201 from the data line after demultiple. chapter4rough/txpbeforesrme somegathers-demul 

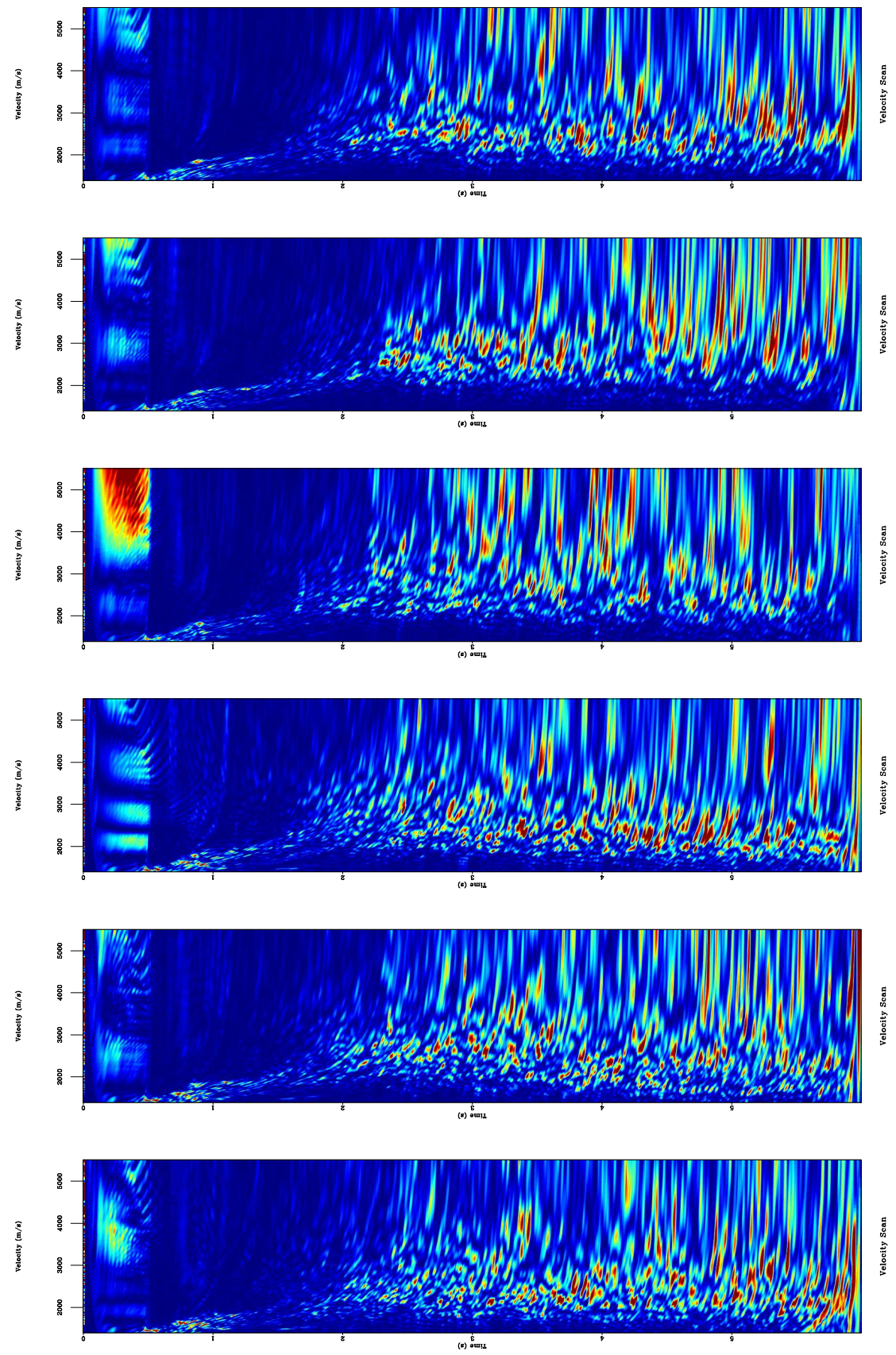

Figure 4.13: Velocity scans for CMPs 201, 401, 601, 801, 1001 and 1201 from the data line after demultiple. chapter4rough/txpbeforesrme vscan-demul 


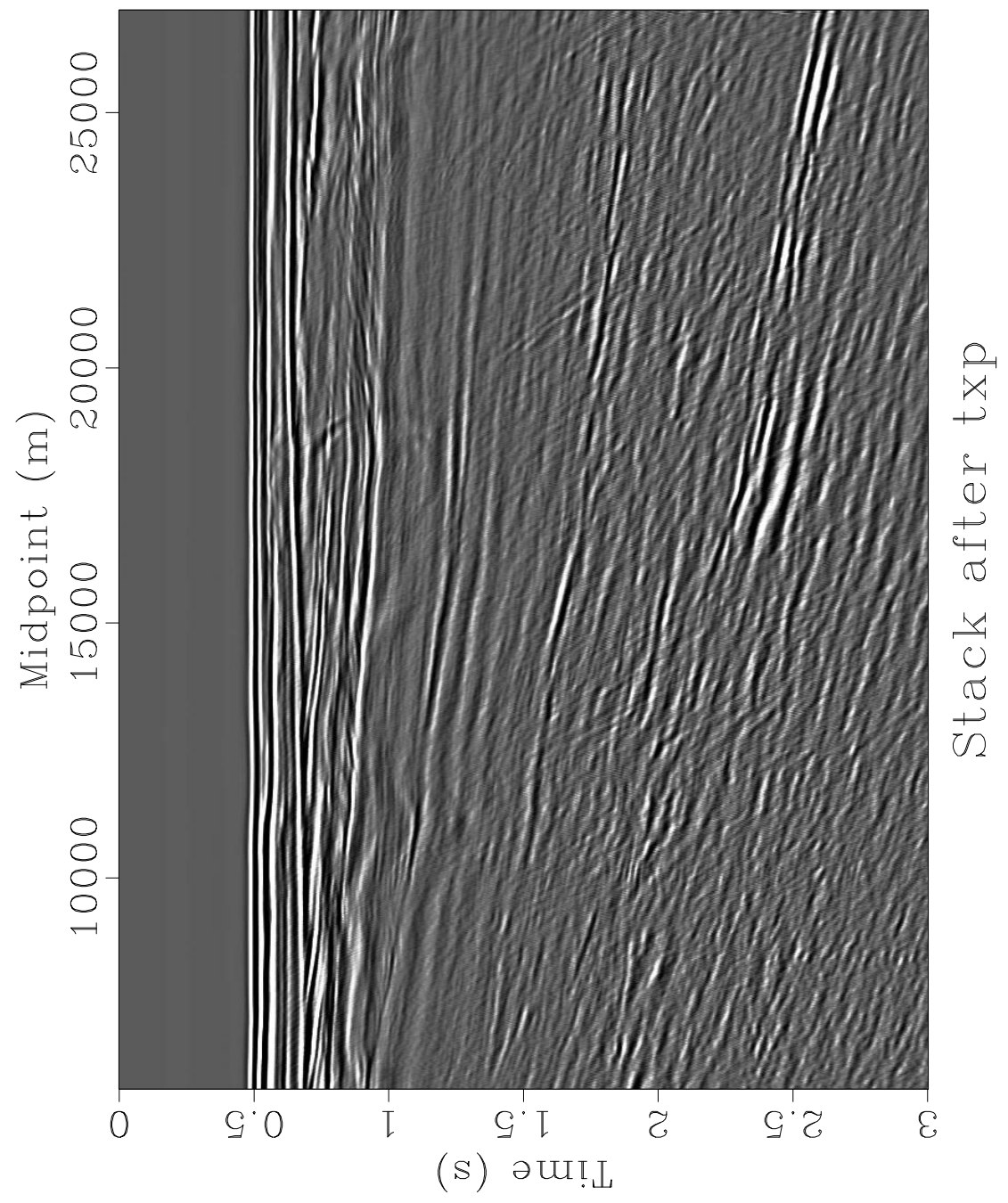

Figure 4.14: Normal Moveout (NMO) stack with picked primary velocity for the entire line for the first 3s after demultiple. chapter4rough/txpbeforesrme stack201-2001-demul-3 


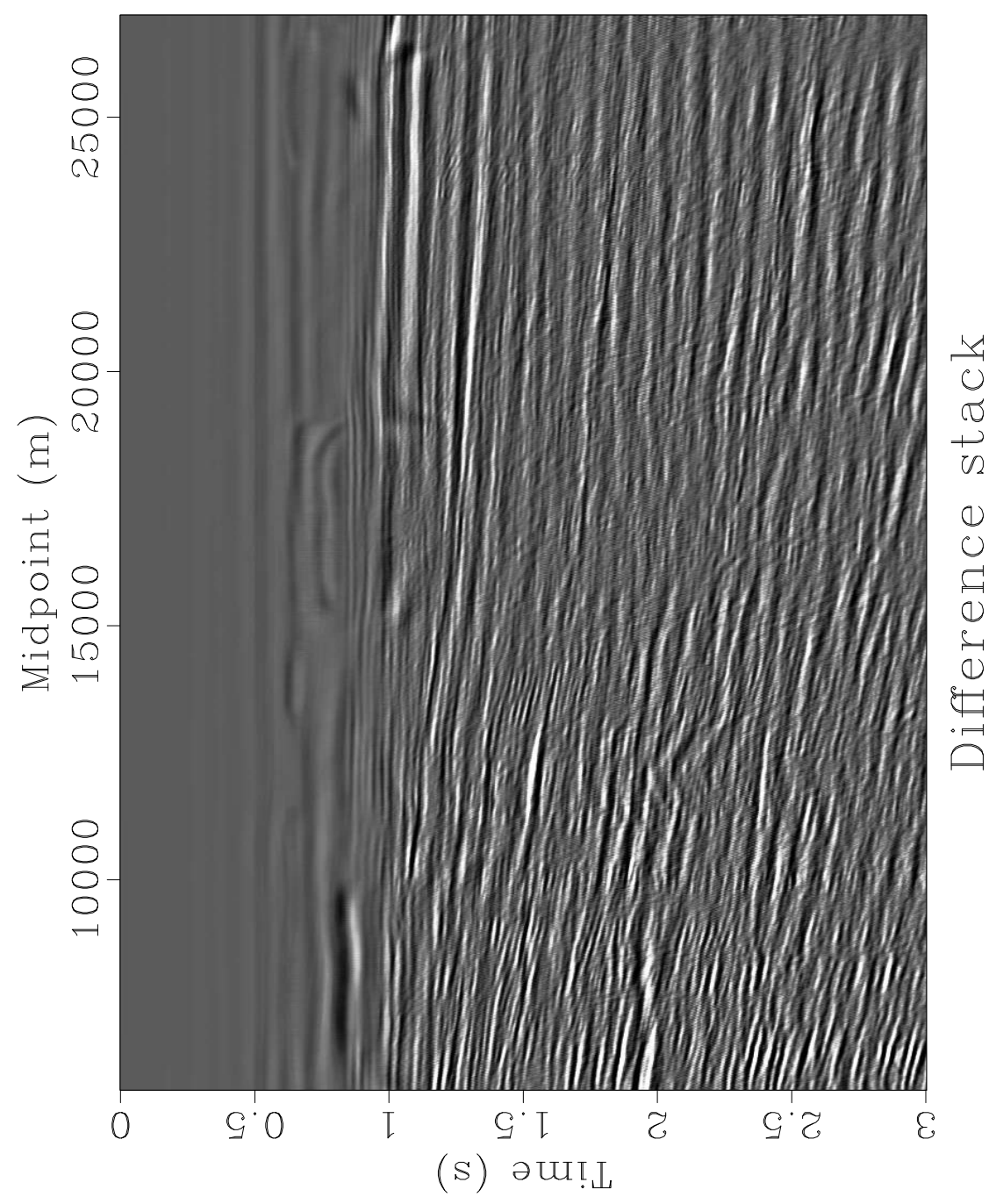

Figure 4.15: Difference stack before and after demultiple in $t-x-p$ domain. chapter4rough/txpbeforesrme stack-difference-3 


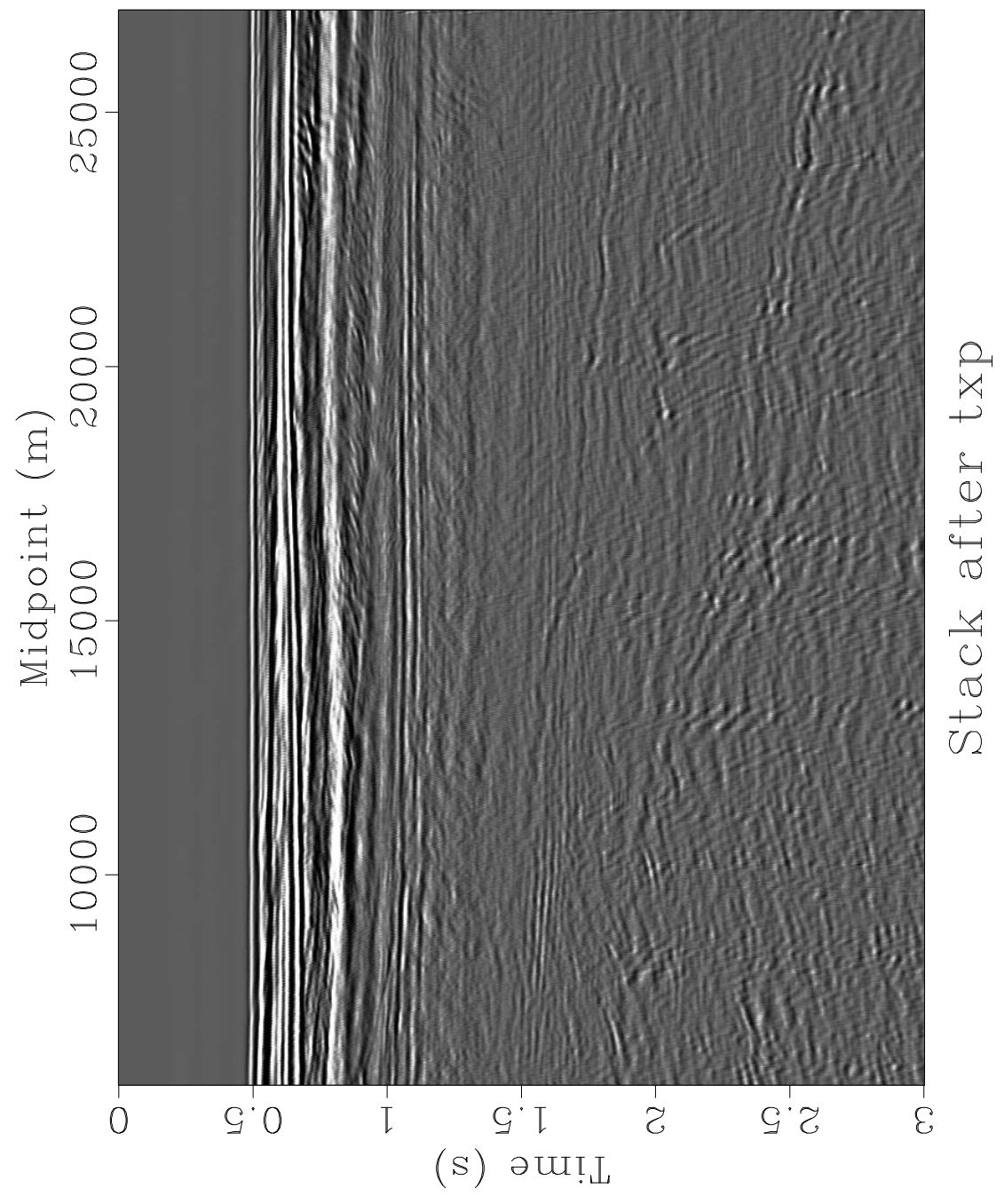

Figure 4.16: Normal Moveout (NMO) stack with water velocity for the entire line for the first 3s after demultiple. chapter4rough/txpbeforesrme stack201-2001-water-demul-3 


\section{Surface Related Multiple Elimination (SRME)}

Since for field data, usually a time procesing work flow includes several demultiple techniques working in conjunction with each other, in this section, I present results obtained using SRME (Dragoset, 1998). In the next section, I show results of filtering in the $t-x-p$ domain after application of SRME to stress on the effectiveness of the combination of two techniques that work well for near-offset (SRME) and mid and far offset ( $t$ - $x$ - $p$ filtering).

Figures 4.17 and 4.18 show the Normal Moveout (NMO) stacks for the first 3s with the picked primary velocities (Figure 4.9) with the same clip before and after demultiple respectively. It is clear that the water bottom multiple in the left corner of the NMO stack in Figure 4.17that appears at around 1s is not present in the stack after SRME. There are other surface related multiples that are also removed that is reflected in the difference section (Figure 4.19 between Figures 4.17 and 4.18. It is clear from Figure 4.19 that an appreciable amount of surface related multiples have been suppressed without killing any significant amount of primaries.

The abovementioned claim is further reflected in the stacks with water velocities before and after SRME. Figures 4.20 and 4.21 show the brute stacks with water velocity for the seismic line before and after application of SRME respectively. It is clear that much of the water bottom multiples that appear from around 1s in Figure 4.20 is clearly suppresed in Figure 4.21

I have claimed earlier that SRME is very effective for near offset. This is reflected by looking at the near offset sections before and after application of SRME in Figures 4.22 and 4.23 that show the near offset sections for the first 3s before and after SRME. It is clear from these figures that the 
application of SRME works very well at near offset as most of the multiples at near-offset have been suppressed by the application of SRME. Figure 4.24 shows the difference section between the near offsets before and after application of SRME.

Figure 4.25 shows velocity scans for CMPs 201, 401, 601, 801, 1001 and 1201 after application of SRME. A comparision with Figure 4.7 shows that the events are more visible in the velocity scans after SRME. This is especially true towards the beginning (CMP 201) of the line, where before any demultiple is done, one can barely see any events after 1s in the velocity scan. 


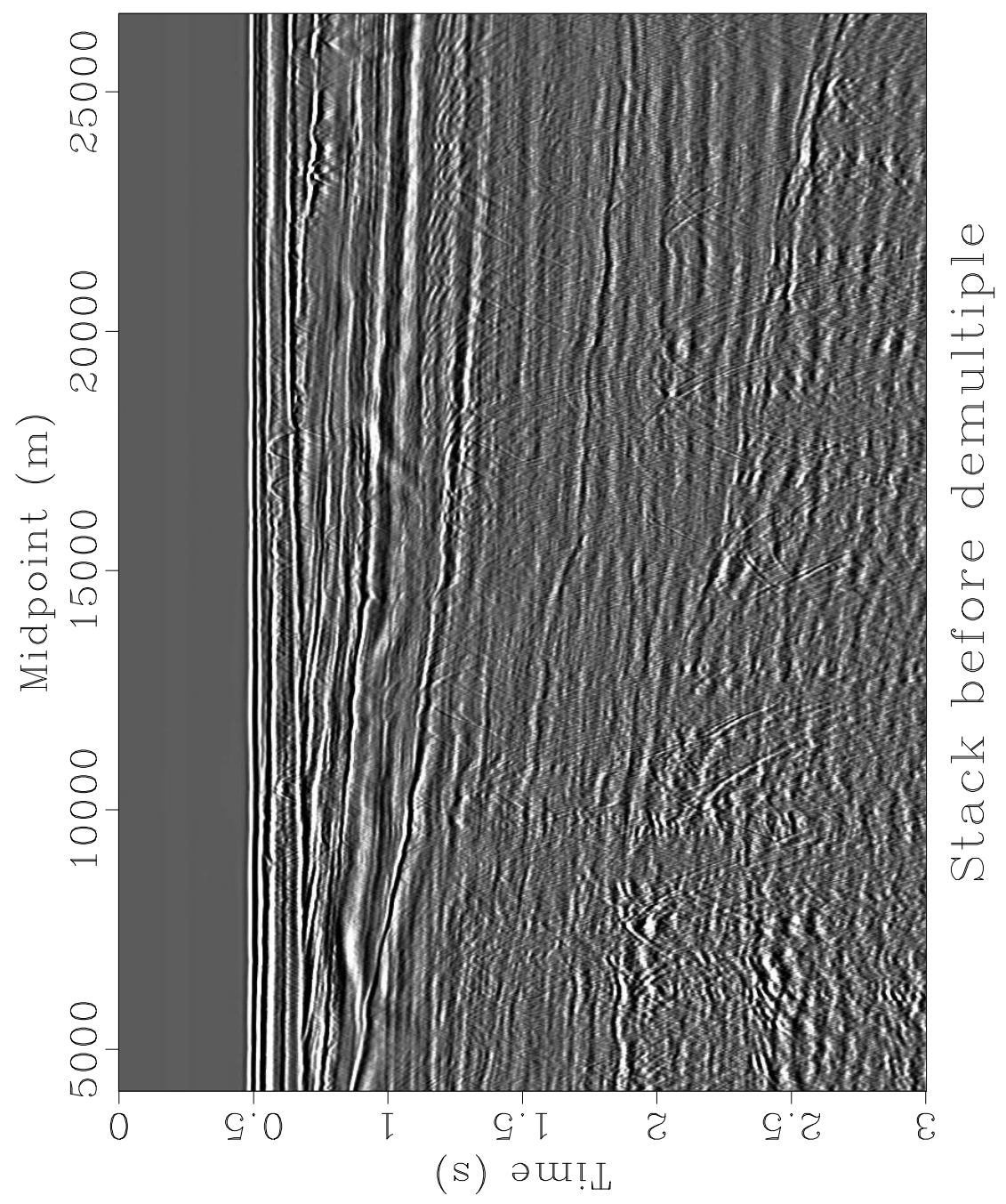

Figure 4.17: Normal Moveout (NMO) stack with picked primary velocity for the entire line for the first 3s before SRME. chapter4rough/srme stackbeforesrme 


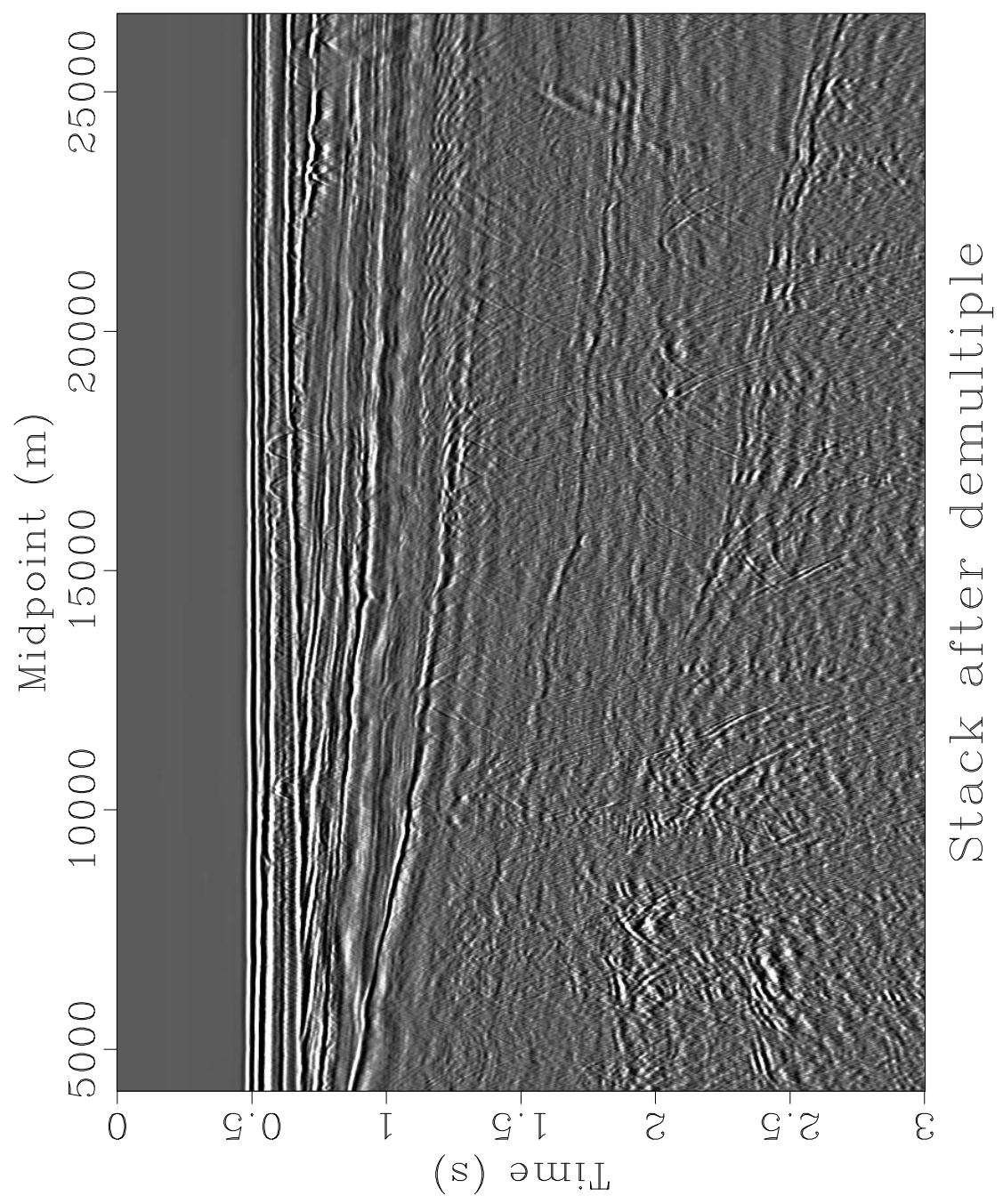

Figure 4.18: Normal Moveout (NMO) stack with picked primary velocity for the entire line for the first 3s after SRME. chapter4rough/srme stackaftersrme 


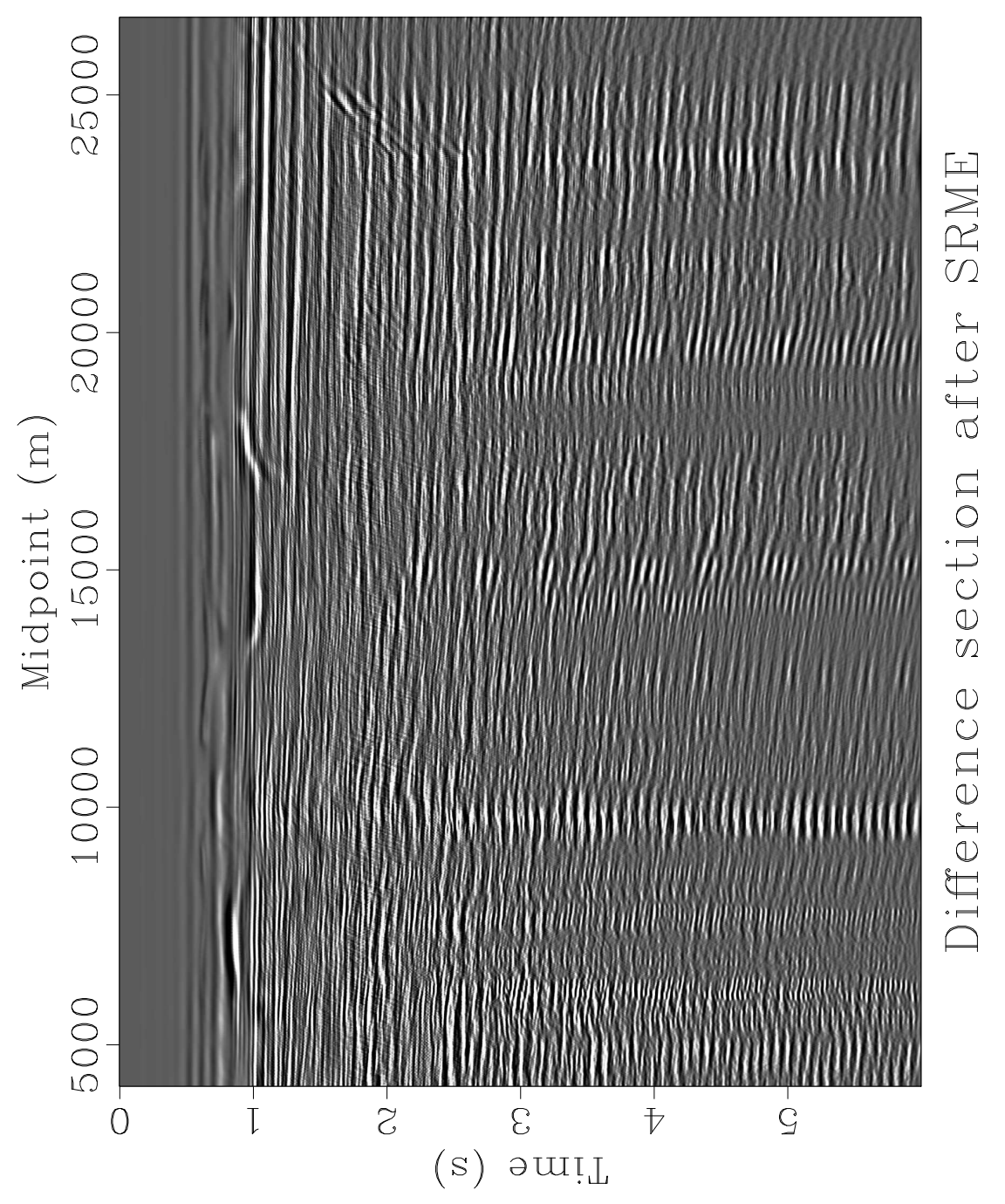

Figure 4.19: Difference section before and after SRME. chapter4rough/srme stackdifferencesrme 


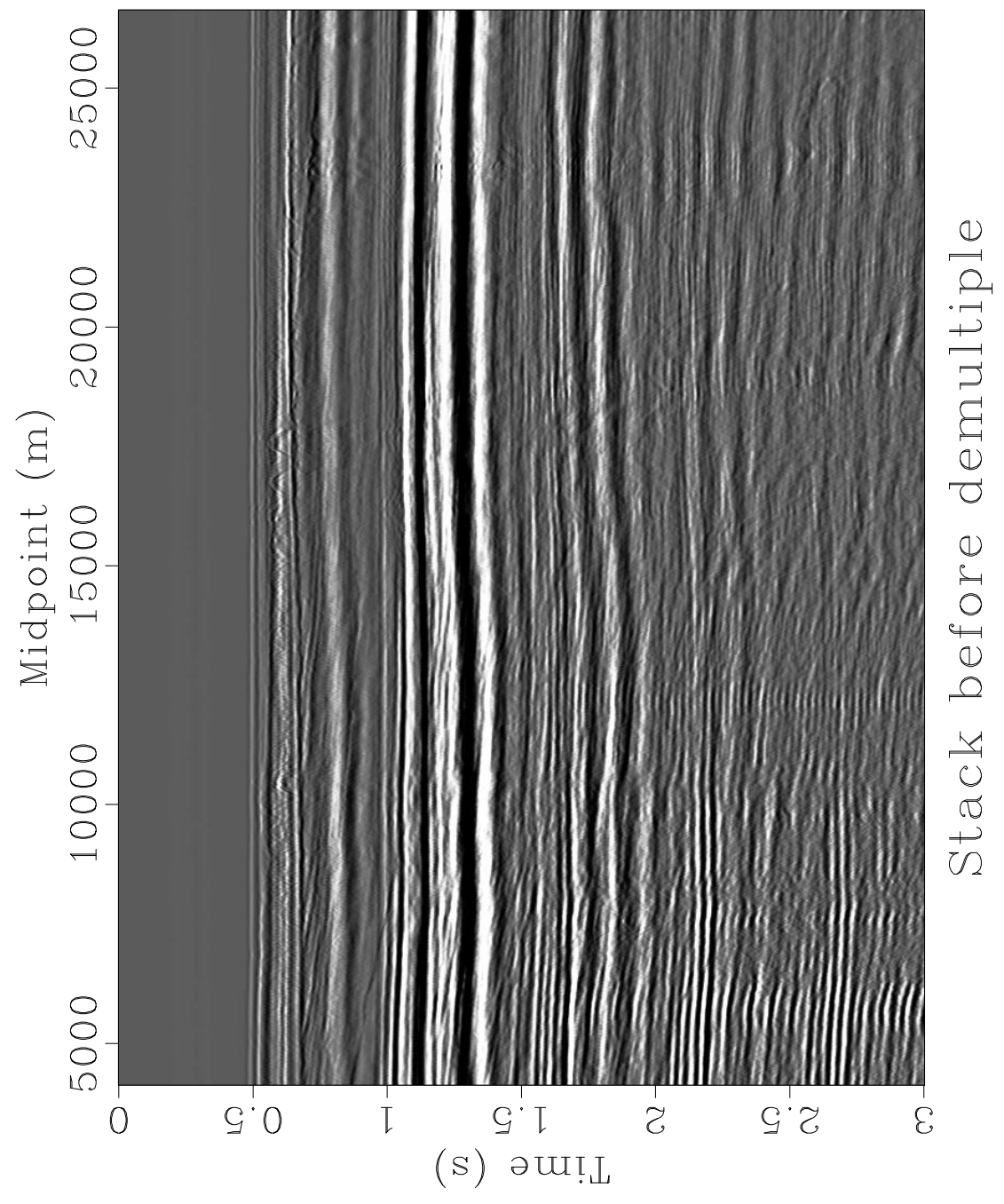

Figure 4.20: Normal Moveout (NMO) stack with water velocity for the entire line for the first 3s before demultiple. chapter4rough/srme stackwaterbeforesrme 


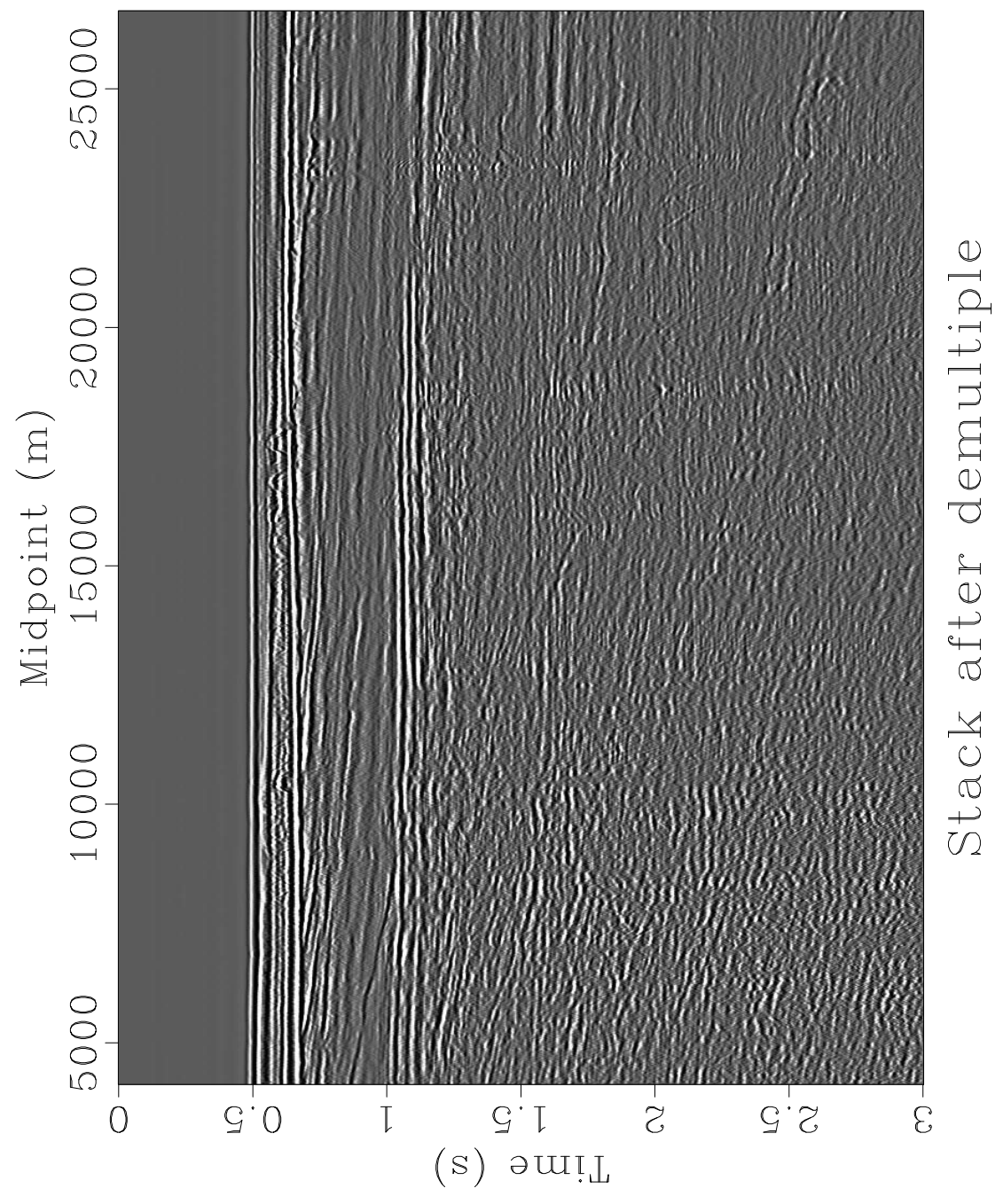

Figure 4.21: Normal Moveout (NMO) stack with water velocity for the entire line for the first 3s after SRME. chapter4rough/srme stackwateraftersrme 


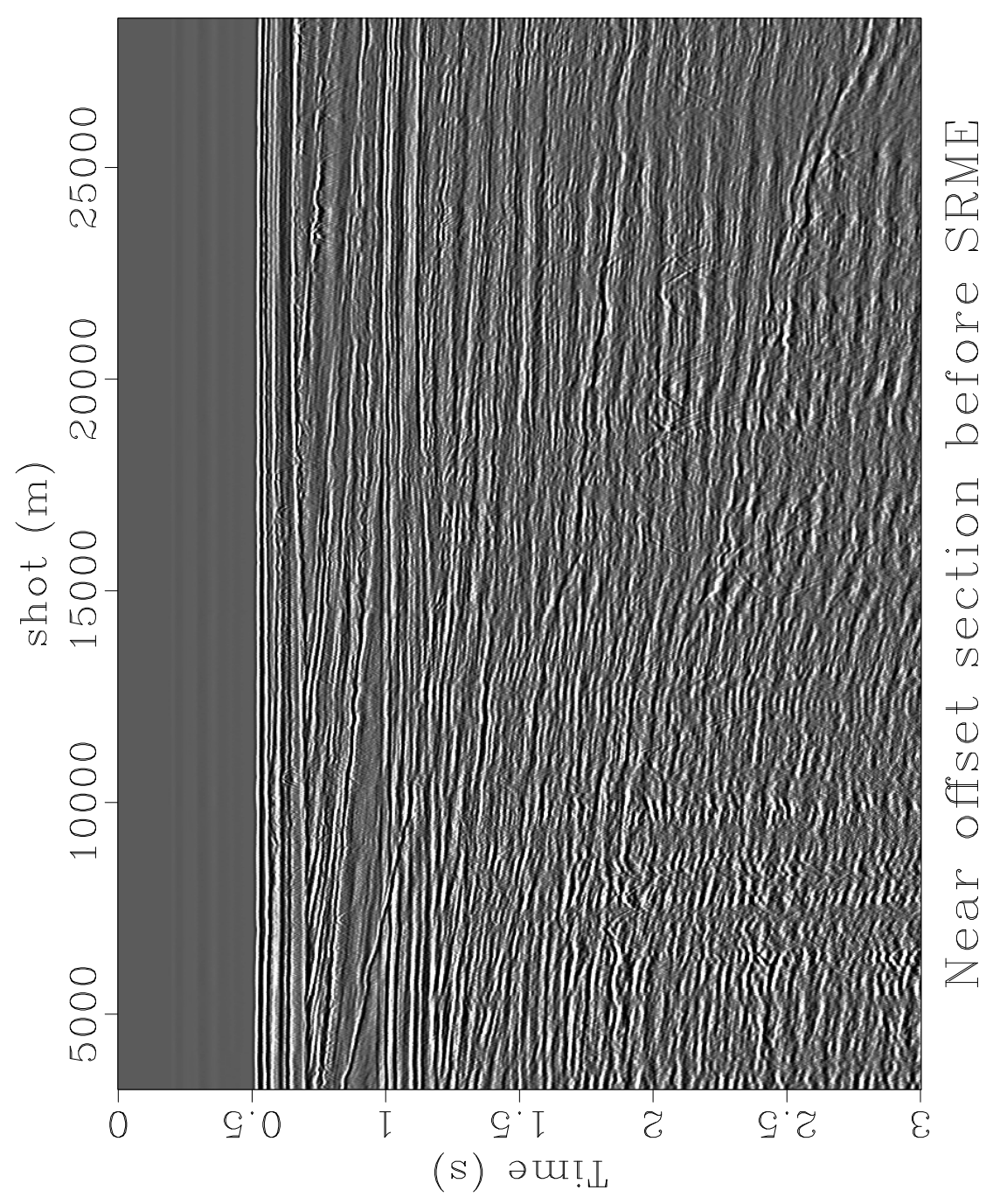

Figure 4.22: Near offset section before SRME. chapter4rough/srme nobeforesrme2 


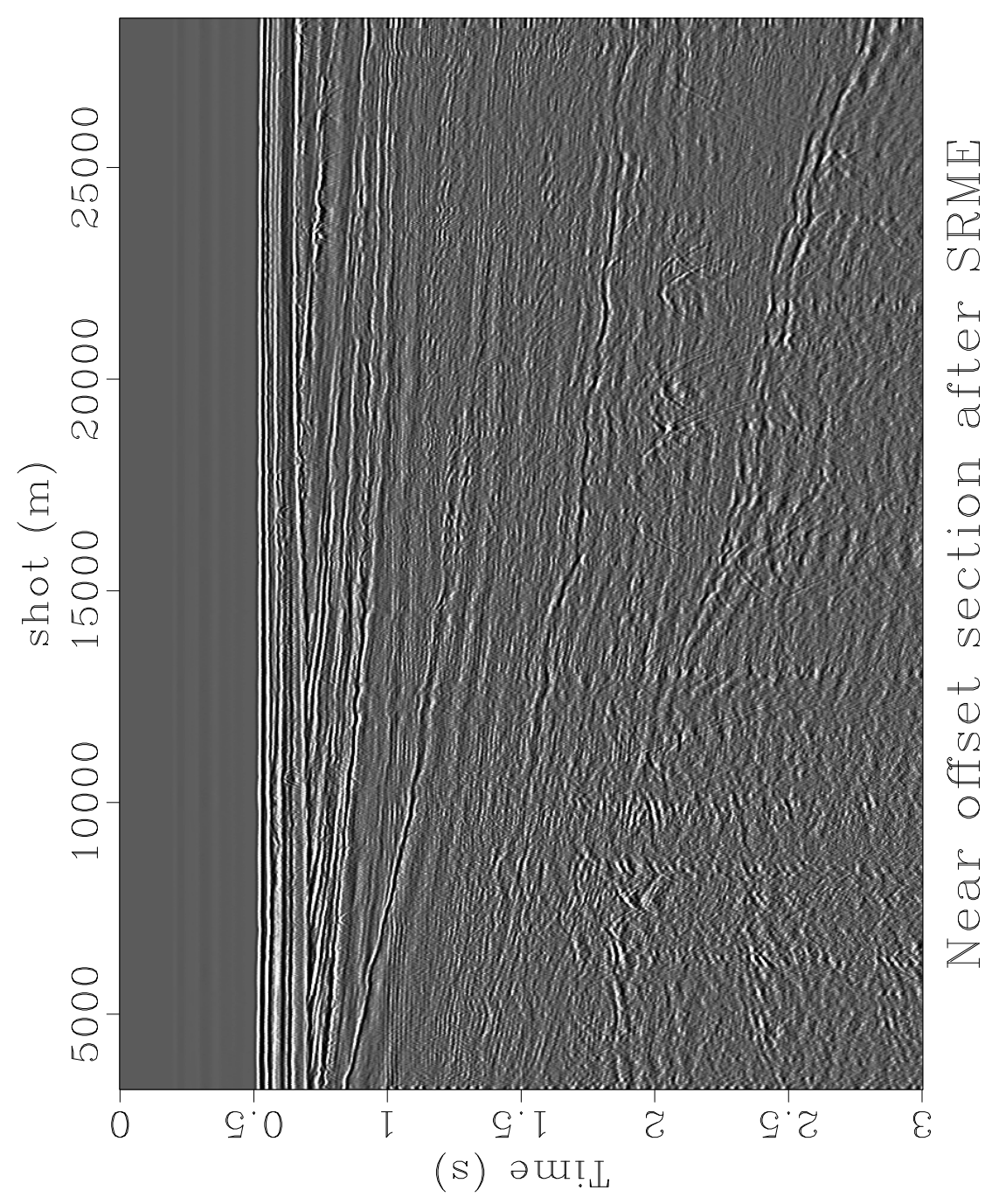

Figure 4.23: Near offset section after SRME. chapter4rough/srme noaftersrme2 


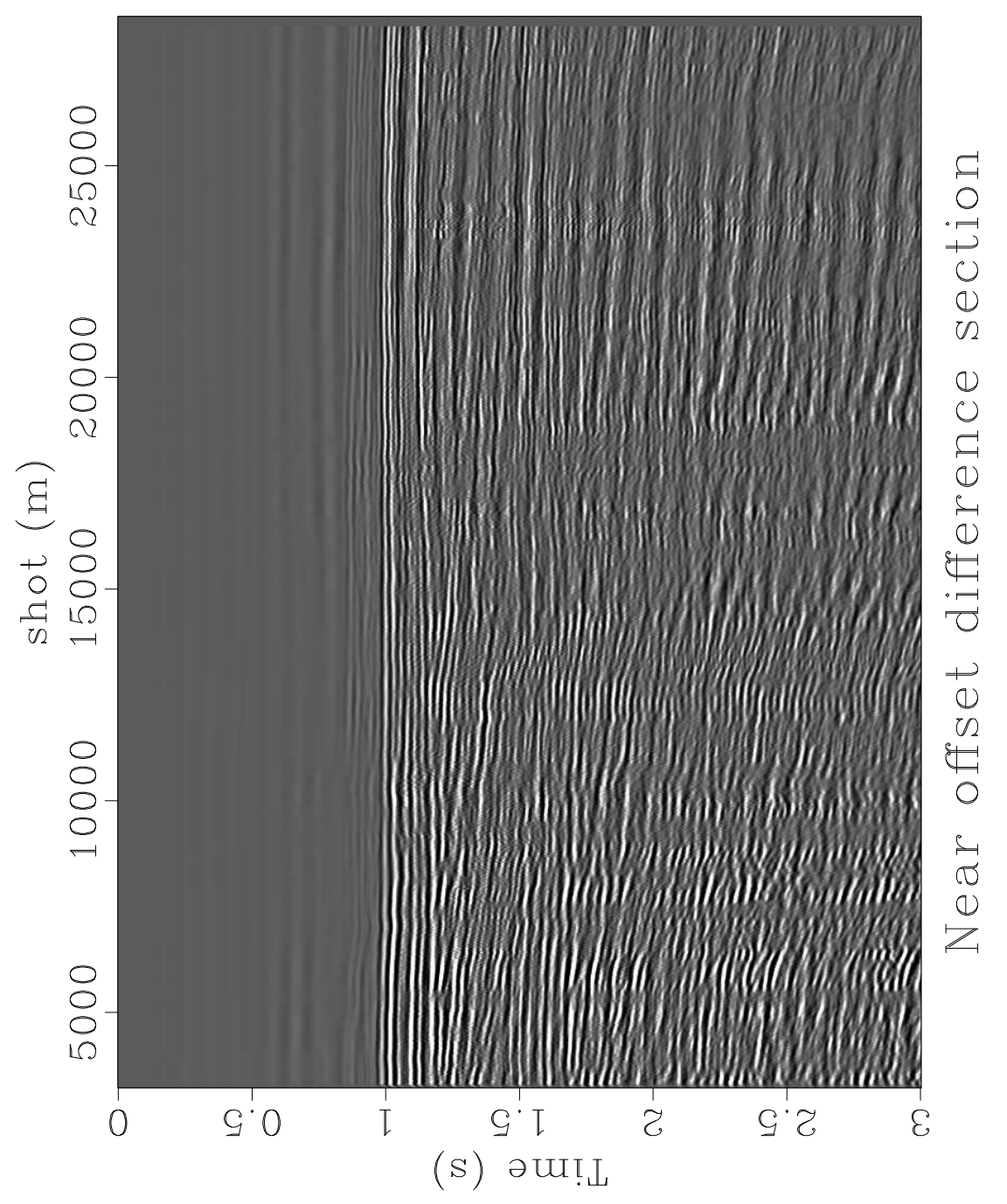

Figure 4.24: Near offset difference section after SRME. chapter4rough/srme differenceno2 

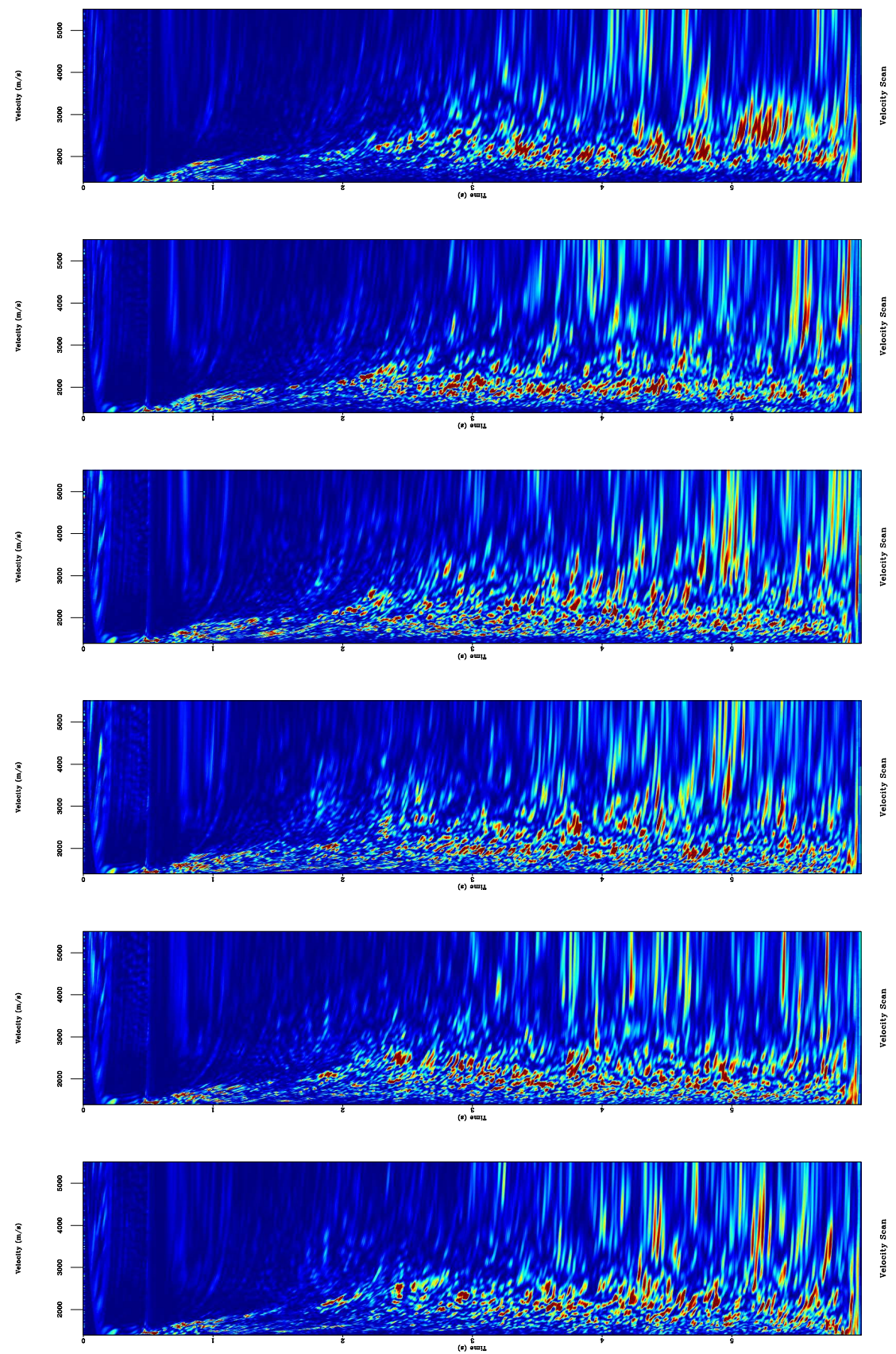

Figure 4.25: Velocity scans for CMPs 201, 401, 601, 801, 1001 and 1201 after SRME. chapter4rough/txpmild vscansbefore 


\section{Surface related multiple elimination followed by $t-x-p$ filtering}

After application of SRME, I applied filtering in the $t-x-p$ domain with mild filter coefficients to preserve as much primaries as possible while attenuating multiples. Figure 4.26 shows CMPs 201, 401, 601, 801, 1001 and 1201 before $t-x$ - $p$ filtering and Figure 4.27 shows the same CMPs after $t-x-p$ filtering. A comparison between the two figures clearly shows much cleaners gathers after $t-x-p$ filtering. Much of the multiples at far offset and some other noise appear to be have been suppressed.

Figures 4.28 and 4.29 shows NMO stacks for the first 3s of data after SRME and after $t-x-p$ filtering. While these 2 sections do not show much difference, a look at the difference section between Figures 4.28 and 4.29 (Figure 4.30 shows that some water bottom multiples starting from around 1s has been removed. It also shows that the leakage of primaries is also minimal. A look at brute stacks (stacks with water velocity) before (Figure 4.31) and after (Figure 4.32) $t-x-p$ filtering shows that filtering in the $t-x-p$ domain eliminates a lot of multiples that are generated from the water column, as well as other kinds of multiples and random noise. It is also worthwhile to note that the two dipping primary reflections that are not distinguishable in Figure 4.31 become apparent in Figure 4.32. This serves as a further indication that multiples have been well suppressed.

The abovementioned claim is further illustrated by the velocity scans of CMP gathers 201, 401, 601, 801, 1001 and 1201 after $t-x-p$ filtering (Figure 4.33p. A comparison of Figures 4.33 and 4.25 shows that it is much easier to pick a trend for primary velocities (specially up to $3 \mathrm{~s}$ ) after $t-x-p$ filtering than before.

After 3s, there are many internal multiples that appear in the data. It is possible to suppress 
some of these multiples further, so as to generate cleaner velocity scans. However, this would come at the cost of eliminating primaries as well and has therefore been avoided. If we are interested in looking at only stacked sections and geometric disposition of geologic events, then one might as well use the filter coefficients more harshly and kill more multiples. 

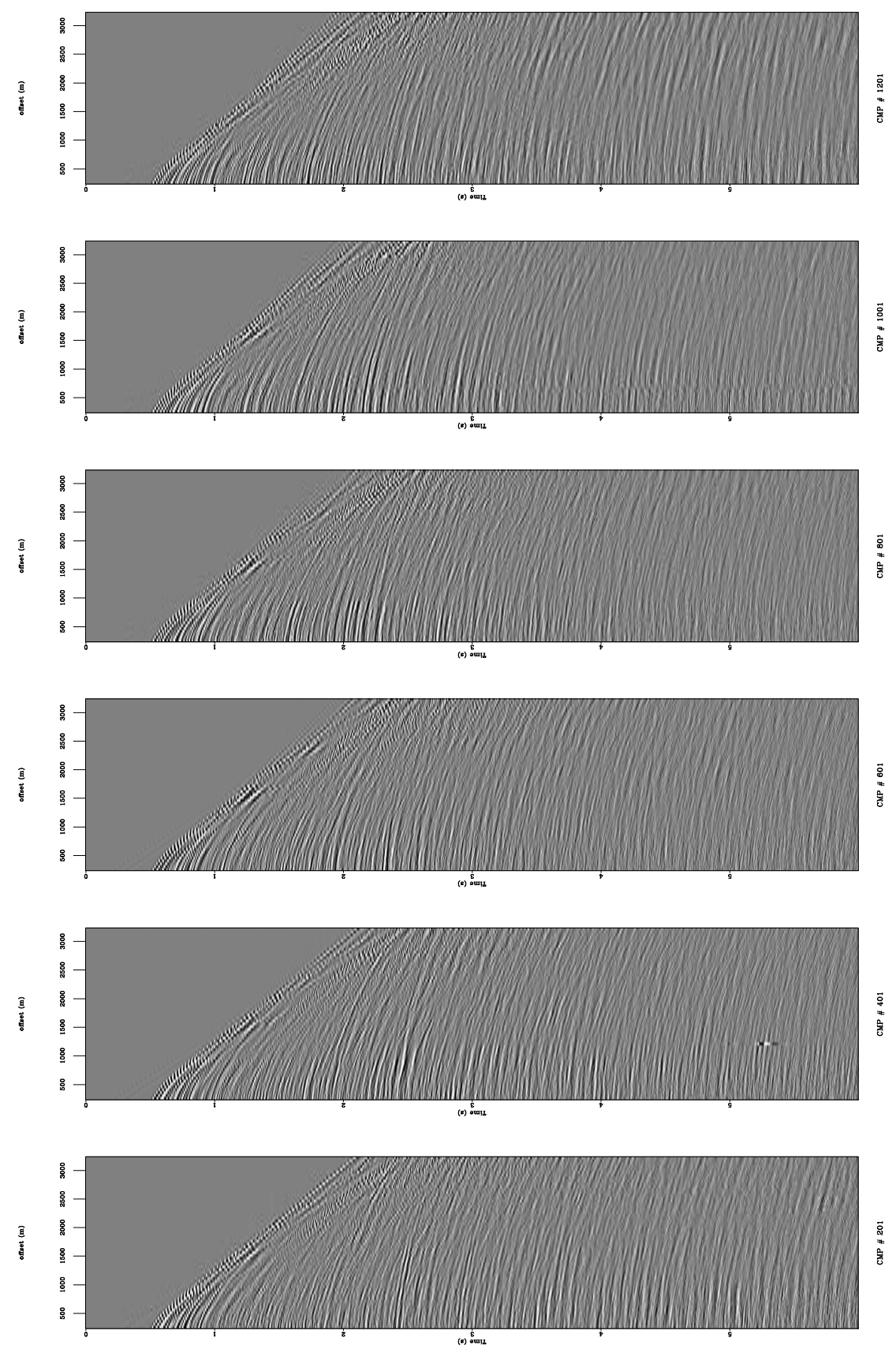

Figure 4.26: CMPs 201, 401, 601, 801, 1001 and 1201 before $t$ - $x$ - $p$ filtering. chapter4rough/txpmild cmpsomebeforetxp 

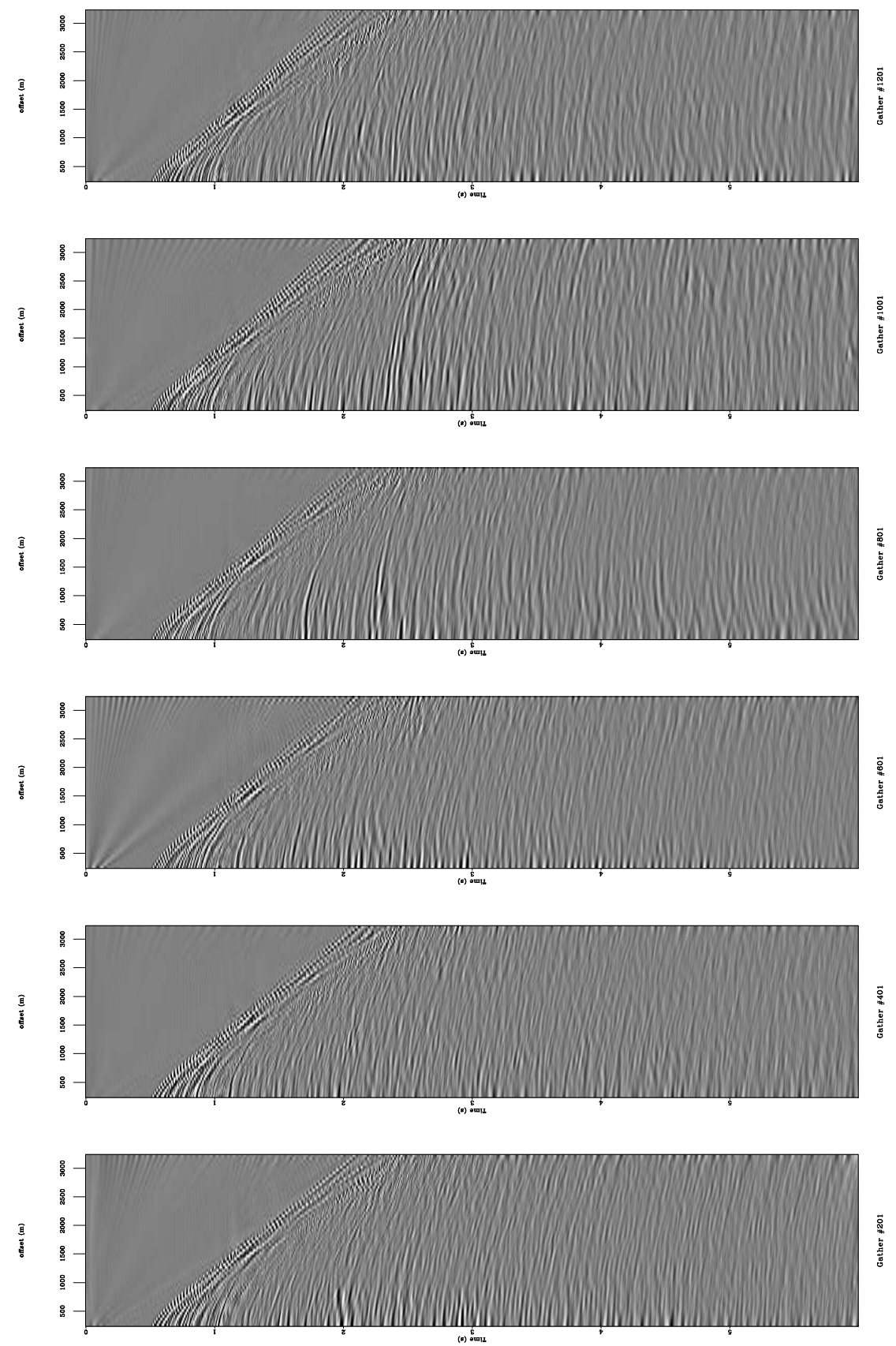

Figure 4.27: CMPs 201, 401, 601, 801, 1001 and 1201 after $t$ - $x$ - $p$ filtering. chapter4rough/txpmildmask cmpsomeaftertxp 


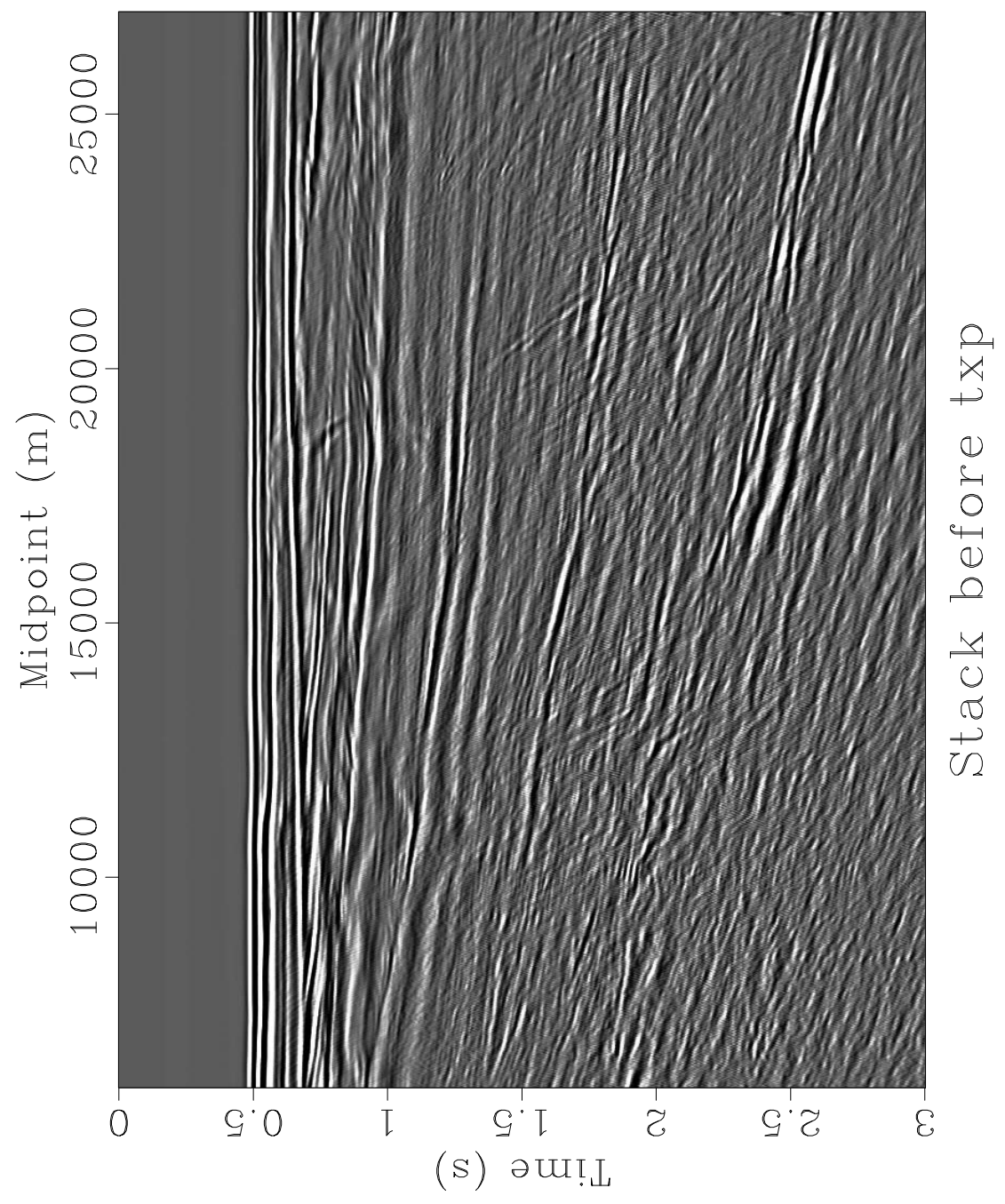

Figure 4.28: Normal Moveout (NMO) stack with picked primary velocity for the entire line for the first 3 s before $t-x-p$ filtering. chapter4rough/txpmildmask stackbeforetxp2 


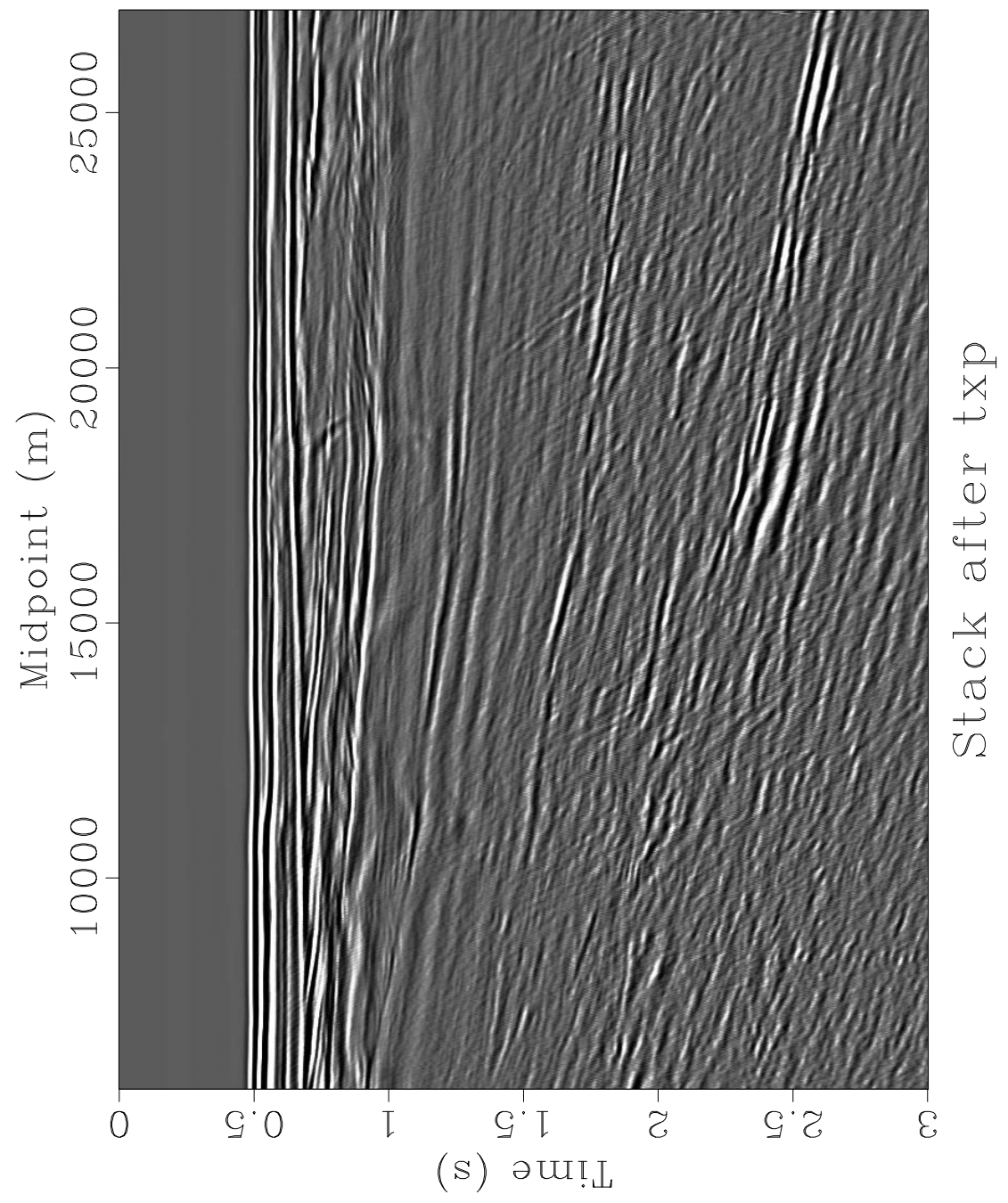

Figure 4.29: Normal Moveout (NMO) stack with picked primary velocity for the entire line for the first 3 s after $t-x-p$ filtering. chapter4rough/txpmildmask stackaftertxp2 


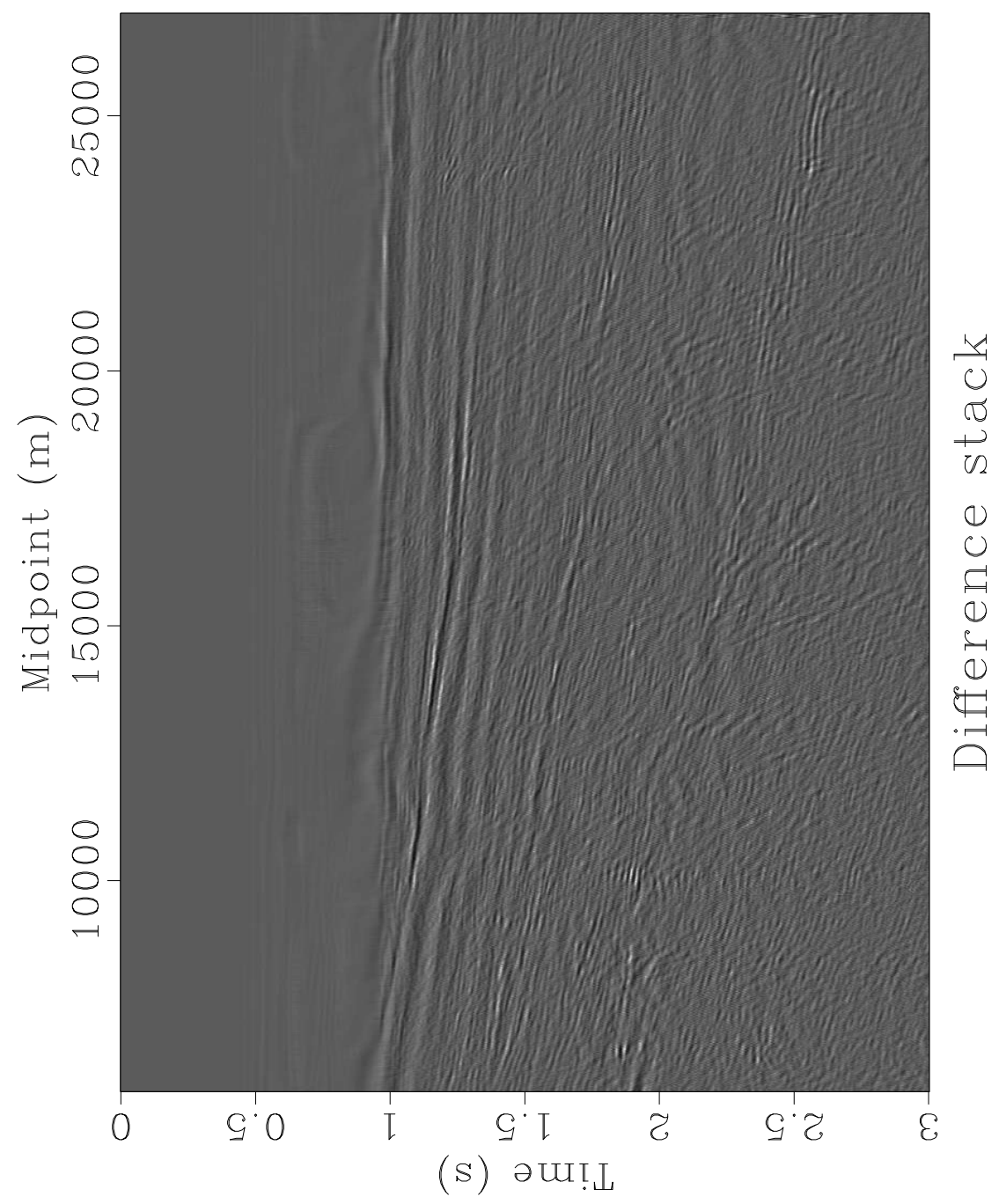

Figure 4.30: Difference section before and after $t-x-p$ filtering. chapter4rough/txpmildmask differencestacktxp2 


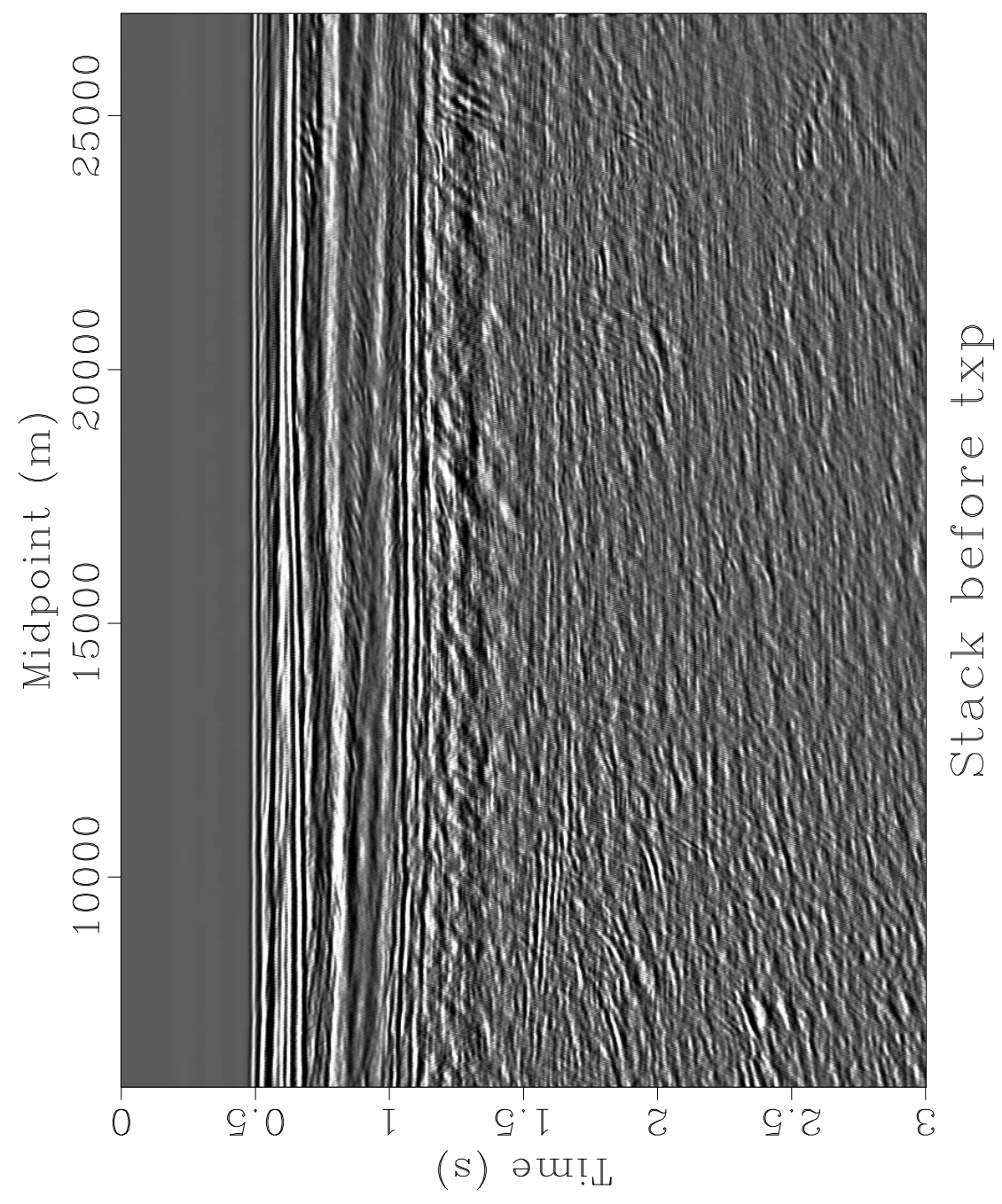

Figure 4.31: Normal Moveout (NMO) stack with water velocity for the entire line for the first 3s before $t-x-p$ filtering. chapter4rough/txpmildmask stackwaterbeforetxp2 


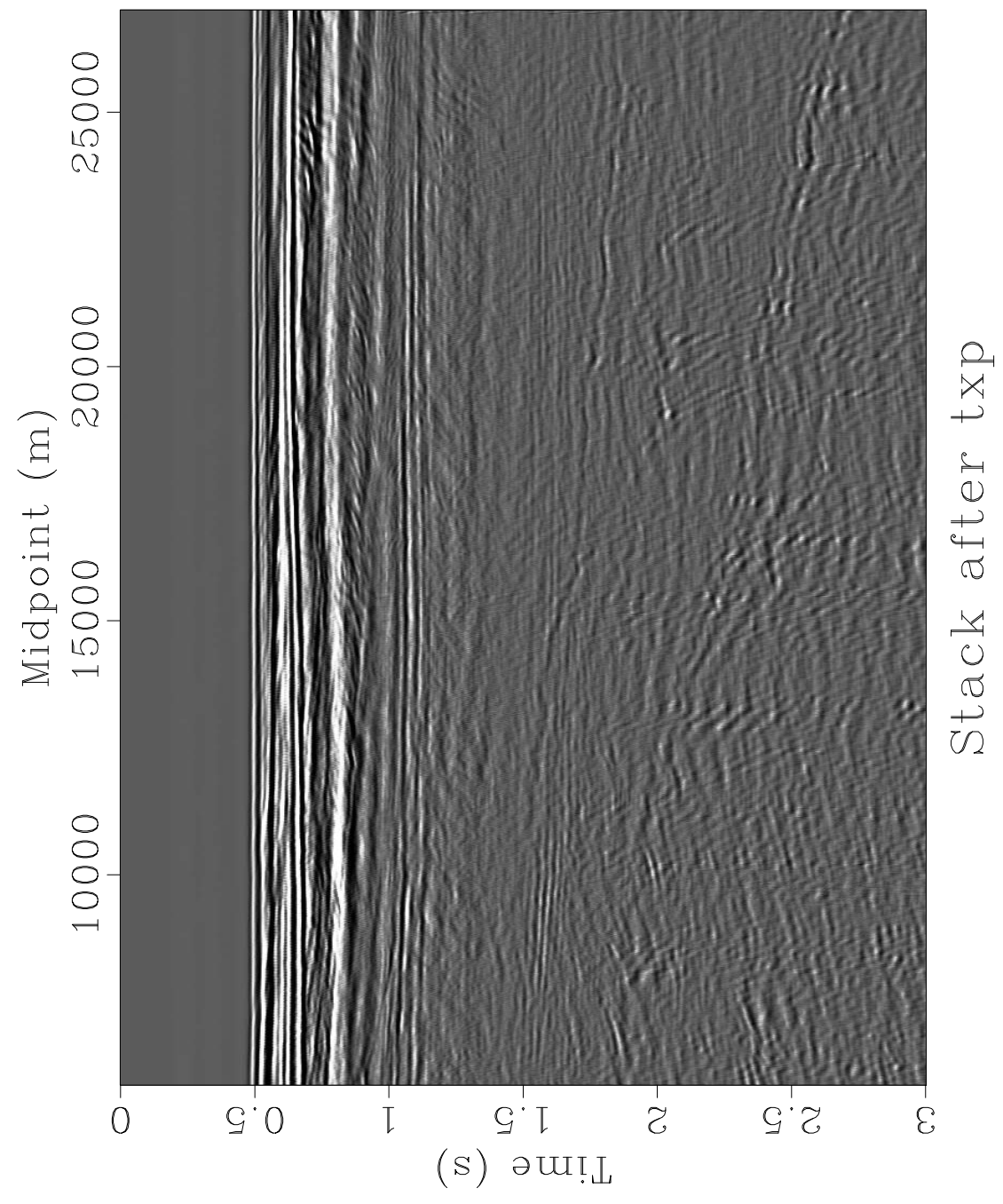

Figure 4.32: Normal Moveout (NMO) stack with water velocity for the entire line for the first 3s after $t-x-p$ filtering. chapter4rough/txpmildmask stackwateraftertxp2 

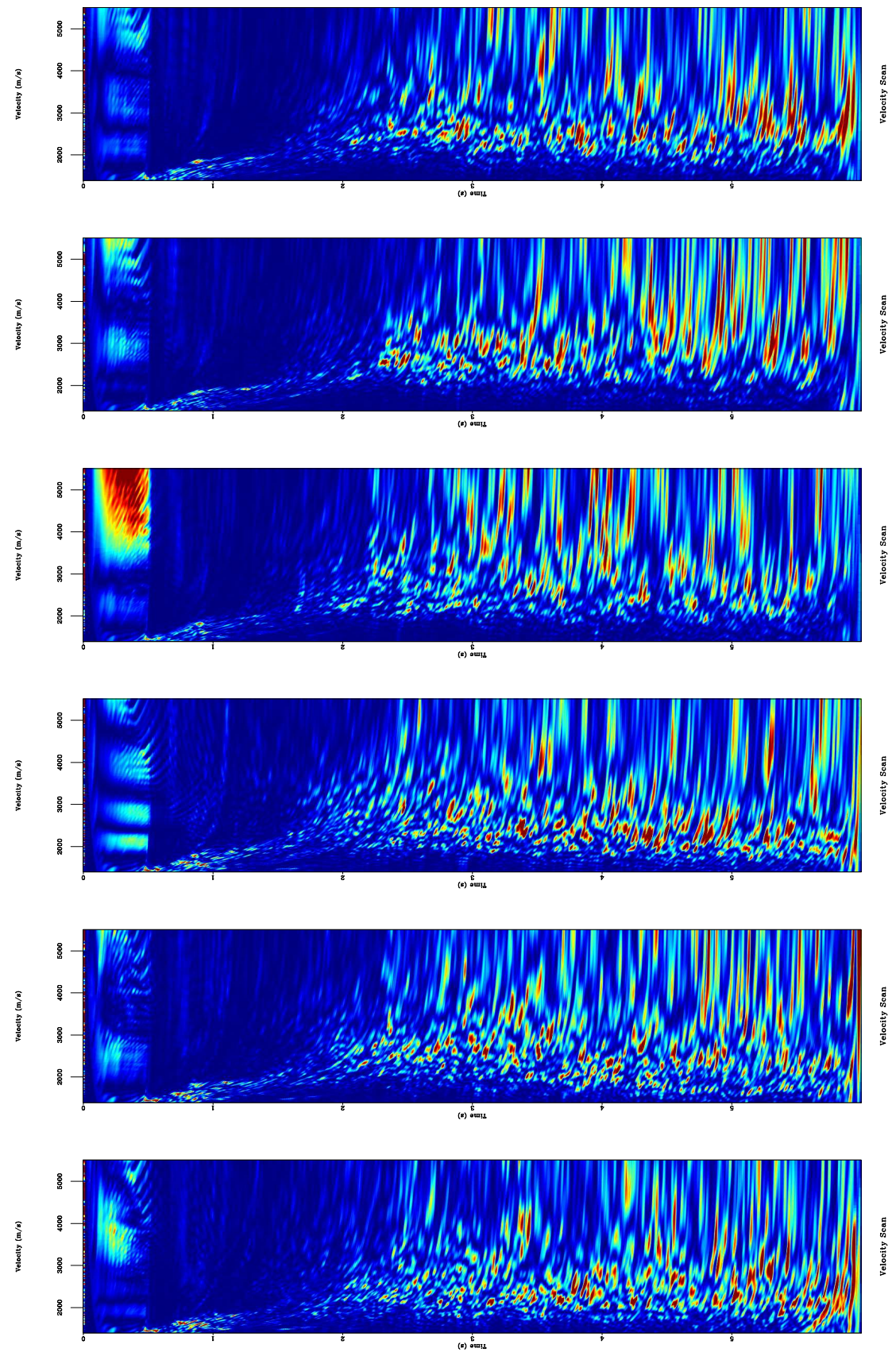

Figure 4.33: Velocity scans for CMPs 201, 401, 601, 801, 1001 and 1201 after $t$ - $x$ - $p$ filtering. chapter4rough/txpmildmask vscansafter 


\section{Chapter 5}

\section{Discussion and conclusions}

This thesis describes the potential of the $t-x-p$ domain for suppression of multiples. Examples demonstrate that this technique can be used to suppress multiples and other kinds of noise which do not follow the hyperbolic paths of primary reflections.

It can be seen from the examples shown in earlier chapters that the new technique can be used effectively for multiple suppression, especially at far offset. At near offset, separation between multiples and primaries remains an issue as there is barely any difference in moveout. However, as in most demultiple workflows used in production, multiple suppression in $t-x-p$ domain can be used very effectively in conjunction with other methods depending on the complexity of the data. For instance, for a shallow water demultiple problem a sequence could be Wave Equation demultiple (WEREM) (Lokshtanov, 2000), followed by Surface Related Multiple Elimination (SRME) (Dragoset, 1998), followed by migration, followed by multiple suppression in $t-x-p$ domain, followed by $f-x$ deconvolution. In such a sequence, WEREM would handle the shallow water demultiple problem where SRME has its own limitations. However, WEREM assumes a simple water bottom and can face problems in areas like a continental shelf where the water bottom no longer remains simple. SRME would help remove multiples in such areas. The velocity filtering in $t-x-p$ domain would help kill multiples at middle and far offset and $f-x$ deconvolution can act as a cosmetic filter to remove any 
remaining multiples and scattering. There is always a balance between killing signal (primaries) and noise (multiples). Care must be taken that if one uses a series of demultiple techniques as part of a time processing worlflow, one preserves primaries while eliminating the maximum amount of multiples possible. WEREM and SRME relies on prediction of multiples which vary in amplitude and phase upto several orders of magnitude from the original gather. The predicted multiples are then adaptively substracted from the data to obtain gathers without multiples. WEREM does the adaptive subtraction in the $\tau-p$ domain. Recent studies have shown that adaptive subtraction for SRME in the wavelet and curvelet domains respectively can produce superior results than in the common offset domain that is used conventionally (Ahmed, 2007, Herrmann et al., 2008).

The multiple suppression technique relies on comparison of intrinsically calculated velocities in the $t_{0}-x-p$ domain to the picked primary velocities. The picked primary velocities need not be absolutely accurate. Reasonably accurate velocites are good enough to yield acceptable results. Since ideally this method should be used in conjunction with WEREM and/or SRME, by the time we use this velocity filtering, we might be able to see a decent trend for primaries in the velocity scan. However, if there are any doubts, one should preferably opt for a slightly higher velocity. The filter coefficients are robust enough to deal with uncertainties in picked primary velocities. This is one of the reasons I use an inverse of an even power in the filter coefficients. This enables us to deal with small numbers that can be controlled with only one large coefficient.

Multiple suppression in $t-x-p$ domain has some specific advantages. For instance, it can be argued that the separation between primaries and multiples is better in this domain as compared to the $\tau$ - $p$ domain if parabolic Radon transform is used. Moreover, in order to go from the $t-x-p$ domain 
to the $t-x$ domain, one does not need to perform inversions as in the case of the hyperbolic Radon transform. A simple stacking operation along the $p$ axis restores the gather in the $t-x$ domain. Alternately, one can go to the $\tau$-p domain by converting the $t$ axis to $\tau$ using the relation $\tau=t-p X$ and then stacking along the $x$ axis. One disadvantage of the method is that we need additional temporary storage space for the third dimension after decomposition of the gather from $t-x$ to the $t-x-p$ domain. 


\section{Bibliography}

Abbad, B., B. Ursin, and M. J. Porsani, 2011, A fast, modified parabolic Radon transform: Geophysics, 76, V11-V24.

Ahmed, I., 2007, 2D Wavelet transform domain adaptive subtraction for enhancing 3d srme: 77th Annual International Meeting, SEG, Expanded Abstracts, 2490-2494.

Alam, A., and J. Austin, 1981, Suppression of multiples using slant stacks: 51st Annual International Meeting, SEG, Expanded Abstracts, 3225-3257.

Askari, R., and H. Siahkoohi, 2008, Ground roll attenuation using the S and x-f-k transform: Geophysical Prospecting, 105-114.

Backus, M., 1959, Water reverberations, their nature and elimination: Geophysics, 24, 233-261.

Berkhout, A., ed., 1982, Seismic imaging: Imaging of wavefield energy by wave field extrapolation, a. theoretical aspects: Elsevier Science Publ. Co., Inc.

Beylkin, G., 1987, Discrete Radon transform: IEEE Trans. Acoust. Speech, Signal Processing, 35, $162-172$.

Claerbout, J. F., 1976, Fundamentals of geophysical data processing: McGraw-Hill Book Co.

Dix, C. H., 1955, Seismic velocities from surface measurements: Geophysics, 20, 68-86.

Dragoset, B., 1998, A practical approach to surface multiple attenuation: 68th Annual International Meeting, SEG, Expanded Abstracts, 1230-1233.

—, 1999, A practical approach to surface multiple attenuation: The Leading Edge, 18(1), 104108.

Fomel, S., 2002, Applications of plane-wave destruction filters: Geophysics, 1946-1960. 
$\longrightarrow$, 2007a, Shaping regularization in geophysical-estimation problems: Geophysics, 72, R29-R36.

—, $2007 \mathrm{~b}$, Velocity-independent time-domain seismic imaging using local event slopes: Geophysics, 72, S139-S147.

- 2009, Adaptive multiple subtraction using regularized nonstationary regression: Geophysics, 74, V25-V33.

Foster, D., and C. Mosher, 1992, Suppression of multiples using the Radon transform: Geophysics, $\mathbf{5 7}, 386-395$.

Ghosh, S., and S. Fomel, 2012, Multiple suppression in the t-x-p domain: 82nd Annual International Meeting, SEG, Expanded Abstracts, doi: 10.1190/segam2012-1398.1.

Glennie, K. ., 1990, Introduction to the petroleum geology of the North Sea: Blackwell Scientific Publications.

Hampson, D., 1986, Inverse velocity stacking for multiple elimination: J. Can. Soc. Expl. Geophys., 22, $44-55$.

$\ldots, 1987$, The discrete Radon transform: A new tool for image enhancement and noise suppression: 57th Annual International Meeting, SEG, Expanded Abstracts, 141-143.

Herrmann, F., D. . Wang, and D. . Verschuur, 2008, Adaptive curvelet-domain primary-multiple separation: Geophysics, 73(3), A17-A21.

Herrmann, P., T. Mojesky, M. Magesan, and P. Hugonnet, 2000, De-aliased, high-resolution Radon transforms: 70th Annual International Meeting, SEG, Expanded Abstracts, 1953-1956.

Hokstad, K. ., and R. . Sollie, 2003, 3D surface-related multiple elimination using parabolic inversion: 73rd Annual International Meeting, SEG, Expanded Abstracts, 1961-1964.

Keys, R., and D. . Foster, 1998, Comparison of seismic inversion methods on a single real data set.: Society of Exploration Geophysicists. 
Kostov, C., 1990, Toeplitz structure in slantstack inversion: 60th Annual International Meeting, SEG, Expanded Abstracts, 1618-1621.

Liu, G., S. Fomel, and X. . Chen, 2009, Time-frequency characterization of seismic data using local attributes: 79th Annual International Meeting, SEG, Expanded Abstracts, 1825-1829.

— 2011, Time-frequency characterization of seismic data using local attributes: Geophysics, $\mathbf{7 6}$, P23-P34.

Liu, Y., and S. Fomel, 2013, Seismic data analysis using local time-frequency decomposition: Geophysical Prospecting, 74, 516-525.

Lokshtanov, D., 2000, Suppression of waterlayer multiples from deconvolution to wave-equation approach: 70th Annual International Meeting, SEG, Expanded Abstracts, 1981-1984.

Lumley, D., D. Nichols, and T. Rekdal, 1995, Amplitude-preserved multiple suppression: 65th Annual International Meeting, SEG, Expanded Abstracts, 1460-1463.

Luo, Y., E. Wang, and N. AlBinHassan, Spring, 2006, Computation of dips and azimuths with weighted structure-tensor approach: Saudi Aramco Journal of Technology, 54-58.

Madiba, G., and G. McMechan, 2003, Processing, inversion, and interpretation of a 2D seismic data set from the North Viking Graben, North Sea: Geophysics, 68(3), 837-848.

Meek, R., and A. Linvelle, 1993, Removing coherent noise in seismic data through tk or fx filtering: 63rd Annual International Meeting, SEG, Expanded Abstracts, 1177-1180.

Ottolini, R., 1983a, Signal/noise separation in dip space, in SEP-37: Stanford Exploration Project, $143-150$.

— 1983 b, Velocity independent seismic imaging: SEP:Stanford Exploration Project, 37, 59-68.

Robinson, E. A., and S. Treitel, 1980, Geophysical signal analysis: Prentice Hall Inc.

Sacchi, M., and M. Porsani, 1999, Fast high resolution parabolic Radon transform: 69th Annual 
International Meeting, SEG, Expanded Abstracts, SPRO P1.1.

Stoffa, P., P. . Buhl, J. . Diebold, and F. . Wenzel, 1981, Direct mapping of seismic data to the domain of intercept time and ray parameter: A plane wave decomposition: Geophysics, 46(3), $255-267$.

Taner, M., 1980, Lomg-period sea-floor multiples and their suppression: Geophysical Prospecting, 28, 30-48.

Tatham, R., 1984, Multidimensional filtering of seismic data: IEEE, 72, 1357-1375.

Thorson, J., and J. Claerbout, 1985, Velocity stack and slant stochastic inversion: Geophysics, 50, $2727-2741$.

Tikhonov, A., 1962, Solution of incorrectly formulated problems and the regularization method: Soviet Mathematics-Doklady.

Treitel, S., P. Gutowski, and D. Wagner, 1982, Plane-wave decomposition of seismograms: Geophysics, 47, 1375-1401.

Trorey, A., 1961, The information content of the reiber sonogram: Geophysics, 26, 761-770.

vanDehem, E. ., and D. . Verschuur, 2002, 3D surface-related multiple prediction using sparse inversion: experience with field data: 72nd Annual International Meeting, SEG, Expanded Abstracts, 2094-2097.

Ventosa, S., C. Simon, and M. Schimmel, 2012, Window length selection for optimum slowness resolution of the local-slant-stack transform: Geophysics, 77, V31-V40.

Verschuur, D., 2012, Seismic multiple removal techniques; past, present and future: European Association of Geoscientists and Engineers.

Verschuur, D., P. Hermann, N. Kinneging, C. Wapenaar, and A. Berkhout, 1988, Elimination of surface-related multiply reflected and converted waves: 58th Annual International Meeting, SEG, 
Expanded Abstracts, 1017-1020.

Weglein, A., and W. Dragoset, eds., 2005, Multiple attenuation: Geophysics reprint series: Society of Exploration Geophysicists.

Yilmaz, E., 2001, Seismic data analysis, Volume-I: Society of Exploration Geophysicists.

Yilmaz, O., and D. Cumro, 1983, Worldwide assortment of field seismic records: Western Geophysical Co. 